Portland State University

PDXScholar

\title{
Distribution of the Sum of Independent Unity- Truncated Logarithmic Series Variables
}

Russell James Wayland

Portland State University

Follow this and additional works at: https://pdxscholar.library.pdx.edu/open_access_etds

Part of the Mathematics Commons

Let us know how access to this document benefits you.

\section{Recommended Citation}

Wayland, Russell James, "Distribution of the Sum of Independent Unity-Truncated Logarithmic Series Variables" (1970). Dissertations and Theses. Paper 730.

https://doi.org/10.15760/etd.730

This Thesis is brought to you for free and open access. It has been accepted for inclusion in Dissertations and Theses by an authorized administrator of PDXScholar. Please contact us if we can make this document more accessible: pdxscholar@pdx.edu. 
AN ABSTRAC' OF THE THESIS Of Ruscel? James Wayland for the Master of Science in Matnomatics presented May 20,1970 .

Title: Distribution of the Suin of Independent UnityTruncated Logarithmic Series Variables.

APPROVED BY MEMBERS OF THE THESIS COMMITTEE:

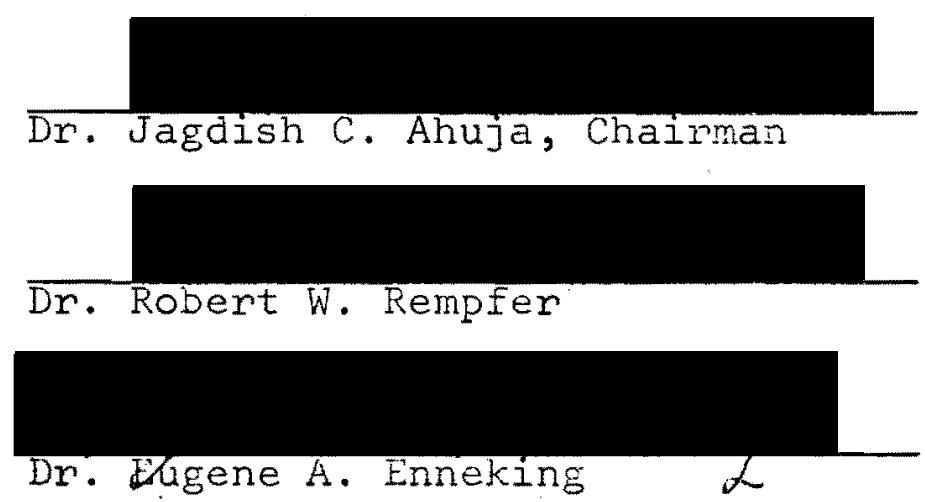

Let $x_{1}, x_{2}, \ldots, x_{n}$ be $n$ independent and identically distributed random variables having the unity-truncated logarithmic series distribution with probability function given by

$$
f(x ; \theta)=\frac{\alpha \theta^{x}}{x} \quad x \in T
$$

where $\alpha=[-\log (1-\theta)-\theta]^{-1}, 0<\theta<1$, and $T=\{2,3, \ldots, \infty\}$. Define their sum as $z=x_{1}+x_{2}+\ldots+x_{n}$.

We derive here the distribution of $Z$, denoted by $p(z ; n, \theta)$, using the inversion formula for characteristic 
functions, in an explicit form in terms of a linear combination of Stirling numbers of the first kind. A recurrence relation for the probability function $p(z ; n, \theta)$ is obtained and is utilized to provide a short table of $p(z ; n, \theta)$ for certain values of $n$ and $\theta$. Furthernore, some properties of $p(z ; n, \theta)$ are investigated following Patil and Wani [Sankhyā, Series A, 27, (1965), 271-280]. 
DISTRIBUTION OF THE SUM OF INDEPENDENT UNITY-TRUNCATED

LOGARITHMIC SERIES VARIABLES

by

RUSSELL JAMES WAYLAND

A thesis submitted in partial fulfillment of the requirements for the degree of

MASTER OF SCIENCE

MATHEMATICS

Portland State University

1970 
TO THE OFFICE OF GRADUATE STUDIES:

The members of the Committee approve the thesis of Russell James Wayland presented May 20, 1970.

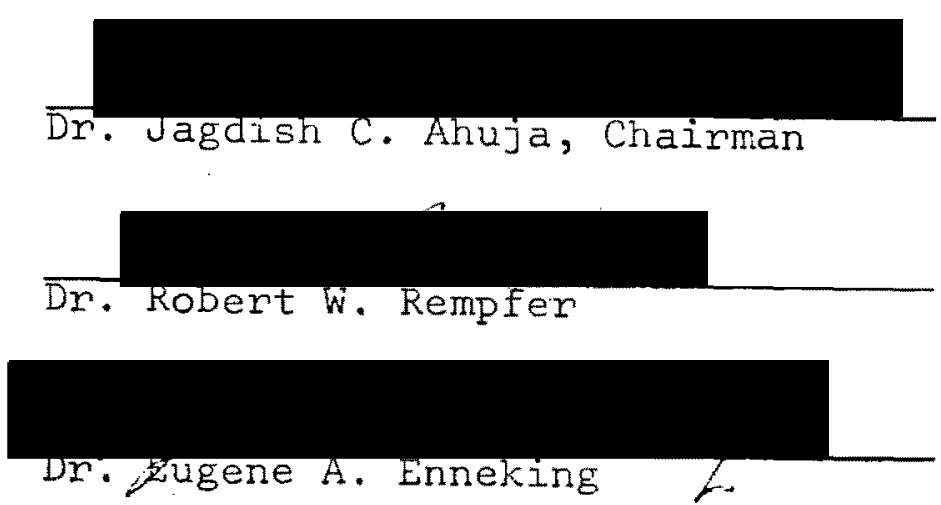

APPROVED :

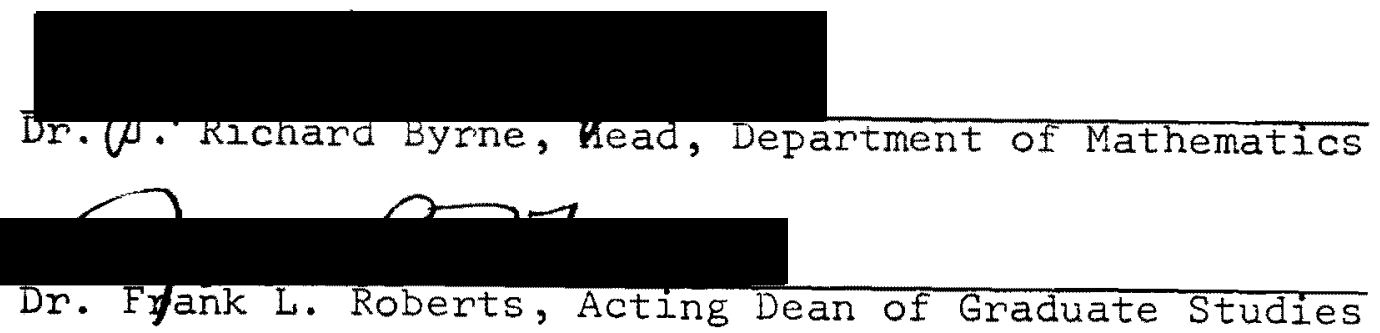

May 21,1970 


\section{ACKNOWLEDGEMENTS}

The author wishes to express his sincere thanks to Dr. Jagdish C. Ahuja of the Department of Mathematics, Portland State University, for suggesting the topic of this thesis, and feels highly indebted to him for his invaluable advice and comments during its preparation. He also wishes to extend his thanks to Mr. Dan Purcell for his valuable help in programming and preparation of the table, and to the computing Center of oregon state University for providing computing facilities. Another expression of gratitude is given by the author to Miss Jeanne Troeh for her many hours spent at the typewriter in the completion of this work. 


\section{TABLE OF CONTENTS}

PAGE

ACKNOWLEDGEMENTS $\ldots \ldots \ldots \ldots \ldots \ldots \ldots \ldots \ldots$

CHAPTER

I INTRODUCTION $\ldots \ldots \ldots \ldots \ldots \ldots \ldots \ldots \ldots$

II DEFINITIONS AND NOTATIONS..........

III DISTRIBUTION OF THE SUM............ 6

IV SOME PROPERTIES OF $p(z ; n, \theta) \ldots \ldots \ldots \ldots$

$V$ TABULATION OF $p(z ; n, \theta) \ldots \ldots \ldots \ldots \ldots \ldots$

BIBLIOGRAPHY $\ldots \ldots \ldots \ldots \ldots \ldots \ldots \ldots \ldots \ldots \ldots \ldots \ldots \ldots$ 
CHAPTER I

\section{INTRODUCTION}

Let $X$ be a random variable having the logarithmic series distribution

$$
g(x ; \theta)=\frac{[-\log (1-\theta)]^{-1} \theta^{x}}{x} \quad x \varepsilon I
$$

where $0<\theta<I$, and $I$ is the set of positive integers. Then, the probability function of the unity-truncated logarithmic series distribution is given by

$$
f(x ; \theta)=\frac{\alpha \theta^{x}}{x} \quad x \in T
$$

where $\alpha=[-\log (1-\theta)-\theta]^{-1}, 0<\theta<1$, and $T=\{2,3, \ldots, \infty\}$. The distribution for the sum of $\mathrm{n}$ independent random variables having the logarithmic series distribution $g(x ; \theta)$ has been obtained by Patil and Wani $[4, p .278]$ which they call the first type Stirling distribution. The purpose of this thesis is to derive the distribution for their sum when the random variables have the unitytruncated logarithmic series distribution defined by $f(x ; \theta)$ above.

To describe briefly the organization of the paper 
we first introduce in Chapter II a few definitions and preliminaries which appear in the paper at one place or another. Chapter III contains the derivation of the distribution for the sum, denoted by $p(z ; n, \theta)$, using the inversion formula for characteristic functions. Here, $p(z ; n, \theta)$ is obtained in an explicit form in terms of a linear combination of stirling numbers of the first kind. In Chapter IV, certain properties of the probability function $p(z ; n, \theta)$ are investigated. In particular, we obtain two recurrence relations for $p(z ; n, \theta)$, one of which is used to generate a short table for the values of $p(z ; n, \theta)$ in Chapter $V$. 


\section{DEFINITIONS AND NOTATION}

In order to allow derivations and proofs to evolve with as little interruption as possible, we state the following definitions:

Definition 1: The Stirling numbers of the first kind $S_{z}^{n}$ are defined by Jordan [3, p. 143] as

$$
x^{(z)}=\sum_{n=1}^{z} S_{z}^{n} x^{n}
$$

where $x^{(z)}=x(x-1)(x-2) \ldots(x-z+1)$ is the factorial power function, and $s_{z}^{0}=0, S_{1}^{l}=1$, and $S_{z}^{n}=0$ for $n>z$. Since the numbers $S_{z}^{n}$ have the same sign as $(-1)^{z-n}$, we may write $\left|S_{z}^{n}\right|=(-1)^{z-n} S_{z}^{n}$.

The Stirling numbers of the first kind have been used by Jordan [3, p. 184] to define the numbers $\mathrm{C}(\mathrm{m}, \mathrm{s})$ as follows:

Definition 2: The numbers $c(m, s)$ are given by

$$
c(m, s)=\sum_{k=m+1}^{2 m-s}(-1)^{k+s}\left(\begin{array}{c}
2 m-s \\
k
\end{array}\right) s_{k}^{k-m}
$$

where $c(m, s)=0$ for $m<s+1, c(1,0)=-1, c(m, 0)=$ $(-1)^{\mathrm{m}} \cdot 1 \cdot 3 \cdot 5 \ldots(2 \mathrm{~m}-1)$, and $c(m, m-1)=(-1)^{\mathrm{m}} \mathrm{m} !$. 
These numbers satisfy the recurrence relation

$$
C(m+1, s)=-(2 m-s+1)[C(m, s)+C(m, s-1)] .
$$

It can be readily seen with the help of (3) and (2) that the sign of $C(m, s)$ is identical with that of $(-1)^{m}$. Therefore, $|\mathrm{C}(\mathrm{m}, \mathrm{s})|=(-1)^{\mathrm{m}} \mathrm{C}(\mathrm{m}, \mathrm{s})$. Subsequently, a corresponding definition for the absolute value of the numbers $\mathrm{C}(\mathrm{m}, \mathrm{s})$ would be

$$
|C(m, s)|=\Sigma_{k=m+1}^{2 m-s}(-1)^{k+s} \cdot\left(\begin{array}{c}
2 m-s \\
k
\end{array}\right)\left|s_{k}^{k-m}\right|
$$

and the recurrence relation (3) becomes

$$
|c(m+1, s)|=(2 m-s+1)[|C(m, s)|+|c(m, s-1)|]
$$

A probability distribution which arises from the sum of $\mathrm{n}$ independent random variables, each having the logarithmic series distribution with the same parameter $\theta$, has been defined by Patil [5, p. 37] in terms of the numbers $\left|s_{z}^{n}\right|$.

Definition 3: The random variable $Z$ is said to have the first type stirling distribution with parameters $\mathrm{n}$ and $\theta$ if its probability function is given by

(6)

$$
f(z ; n, \theta)=\frac{\beta^{n} n !\left|S_{z}^{n}\right| \theta^{z}}{z !}
$$

for $z=n, n+1, n+2, \ldots \infty, n=1,2,3, \ldots \infty$, where 
$\beta=[-\log (1-\theta)]^{-1}$ and $0<\theta<1$.

The characteristic function of the probability

function $f(z ; n, \theta)$ is easily found to be

$$
\psi_{z}(t)=\beta^{n}\left[-\log \left(1-\theta e^{i t}\right)\right]^{n}
$$

Some of the properties, which we will examine, deal with a large class of probability distributions which is defined by G.P. Patil [5, p. 2] in the following manner.

Definition 4: A discrete random variable $\mathrm{X}$ is said to have a generalized power series distribution with the parameter $\theta$ and range $r$ if its probability function is given by

$$
p(x ; \theta)=\frac{a(x) \theta^{x}}{g(\theta)} \quad x \in T
$$

where $a(x)$ is independent of $\theta$ and is positive for $x \varepsilon T$. $T$ is a countable subset of the set of non-negative integers (reals). The series function $g(\theta)=\Sigma a(x) \theta^{x}$, which will be summed over the entire range $T$, will be positive, finite, and differentiable for $\{\theta \mid 0<\theta<R\}$ where $R$ is the radius of convergence for the series function. 


\section{CHAPTER III}

DISTRIBUTION OF THE SUM

Let $x_{1}, x_{2}, \ldots, x_{n}$ be $n$ independent and identically distributed random variables having the unity-truncated logarithmic series distribution with probability function given by

$$
f(x ; \theta)=\frac{\alpha \theta^{x}}{x} \quad x=2,3, \ldots
$$

where $\alpha=[-\log (1-\theta)-\theta]^{-1}$. and $0<\theta<1$.

The characteristic function of the random variable $x_{j}$ is obtained as

$$
\begin{aligned}
\psi_{x_{j}}(t) & =\Sigma_{x_{j}=2}^{\infty} e^{i t x_{j}} f\left(x_{j} ; \theta\right) \\
& =\Sigma_{x_{j}=2}^{\infty} \frac{a\left(\theta e^{i t}\right)_{j}}{x_{j}} \\
& =a\left[-\log \left(1-\theta e^{i t}\right)-\theta e^{i t}\right] .
\end{aligned}
$$

If we now let $z=x_{1}+x_{2}+\ldots+x_{n}$, then, since the $x_{j}$ 's are independent, the characteristic function of $\mathrm{Z}$ is given by the product of characteristic functions of 
the $x_{j}$ 's. Hence,

$$
\begin{aligned}
\psi_{z}(t) & =\prod_{j=1}^{n} \psi_{x_{j}}(t) \\
& =a^{n}\left[-\log \left(1-\theta e^{i t}\right)-\theta e^{i t}\right]^{n} .
\end{aligned}
$$

We may now find the probability function of $Z$ by using the inversion formula for characteristic functions given by Fisz [2, p. 119]. Since it will depend on two parameters, $n$ and $\theta$, we shall denote it by $p(z ; n, \theta)$. Thus we have

$$
\begin{aligned}
p(z ; n, \theta) & =\frac{1}{2 \pi} \int_{-\pi}^{\pi} e^{-i t z} \psi_{z}(t) d t \\
& =\frac{\alpha^{n}}{2 \pi} \int_{-\pi}^{\pi} e^{-i t z}\left[-\log \left(1-\theta e^{i t}\right)-\theta e^{i t}\right]^{n} d t
\end{aligned}
$$

which, after taking the binomial expansion of the second factor under the integral sign, becomes

$$
\begin{aligned}
p(z ; n, \theta)= & \frac{\alpha^{n}}{2 \pi} \int_{-\pi}^{\pi} e^{-i t z} \\
& \cdot \Sigma_{k=0}^{n}\left(\begin{array}{l}
n \\
k
\end{array}\right)\left(-\theta e^{i t}\right)^{k}\left[-\log \left(1-\theta e^{i t}\right)\right]^{n-k} d t \\
= & \frac{\alpha^{n}}{2 \pi} \Sigma_{k=0}^{n}\left(\begin{array}{l}
n \\
k
\end{array}\right)(-\theta)^{k} \\
& \quad \cdot s_{-\pi}^{\pi} e^{-i t(z-k)}\left[-\log \left(1-\theta e^{i t}\right)\right]^{n-k} d t .
\end{aligned}
$$


To evaluate the integral in (13), we observe from (7) that the quantity $\beta^{n-k}\left[-\log \left(1-\theta e^{i t}\right)\right]^{n-k}$ is the characteristic function of the first type stirling distribution with parameters $n-k$ and $\theta$. So the inversion formula for characteristic functions gives us

(14)

$$
\begin{gathered}
\frac{1}{2 \pi} \int_{-\pi}^{\pi} e^{-i t(z-k)}\left[-\log \left(1-\theta e^{i t}\right)\right]^{n-k} d t= \\
\frac{(n-k) !\left|s_{z-k}^{n-k}\right| \theta^{z-k}}{(z-k) !} .
\end{gathered}
$$

Substituting for the integral from (14) into (13), we obtain the probability function of $z$ in the form

(15) $p(z ; n, \theta)=\frac{\alpha^{n} \Sigma_{k=0}^{n}\left(\begin{array}{l}n \\ k\end{array}\right)(-\theta)^{k}(n-k) !\left|s_{z-k}^{n-k}\right| \theta^{z-k}}{(z-k) !}$

$$
=\frac{\alpha^{n} n ! \theta^{z}}{z !} \Sigma_{k=0}^{n}(-1)^{k}\left(k_{k}^{z}\right)\left|s_{z-k}^{n-k}\right|
$$

But, using (4), we find that

$$
|C(z-n, z-2 n)|=\Sigma_{j=z-n+1}^{z}(-1)^{j+z-2 n}\left(\begin{array}{l}
z \\
j
\end{array}\right)\left|s_{j}^{j-z+n}\right|
$$

which, by letting $k=z-j$, becomes

$$
\begin{aligned}
|c(z-n, z-2 n)| & =\Sigma_{k=0}^{n-1}(-1)^{k}\left(\begin{array}{l}
z \\
k
\end{array}\right)\left|s_{z-k}^{n-k}\right| \\
& =\Sigma_{k=0}^{n}(-1)^{k}\left(\begin{array}{l}
z \\
k
\end{array}\right)\left|s_{z-k}^{n-k}\right|
\end{aligned}
$$


since $\left|s_{z-n}^{0}\right|=0$.

Hence, replacing the summation in (15) by (16), we have the following:

Theorem 1: Let $\mathrm{x}_{1}, \mathrm{x}_{2}, \ldots, \mathrm{x}_{\mathrm{n}}$ be $\mathrm{n}$ independent and identically distributed random variables having the unity-truncated logarithmic series distribution defined by $(9)$, and let $z=x_{1}+x_{2}+\ldots+x_{n}$. Then the distribution of $\mathrm{Z}$ is given by

$$
p(z ; n, \theta)=\frac{\alpha^{n} n !}{z !}|c(z-n, z-2 n)| \theta^{z}
$$

for $z=2 n, 2 n+1, \ldots, \infty$, where $\alpha=[-\log (1-\theta)-\theta]^{-1}$

and $0<\theta<1$. 


\section{CHAPTER IV}

SOME PROPERTIES OF $p(z ; n, \theta)$

Some of the more important aspects of $p(z ; n, \theta)$ are examined here as properties and theorems.

Property 1: The first three moments about the mean for the probability function $p(z ; n, \theta)$ with parameters $\mathrm{n}$ and $\theta$ are

$$
\begin{aligned}
& \mu_{1}=\frac{n \alpha \theta^{2}}{1-\theta} \\
& \mu_{2}=\frac{n \alpha \theta^{2}}{(1-\theta)^{2}}\left[2-\theta-\alpha \theta^{2}\right] \\
& \mu_{3}=\frac{n \alpha \theta^{2}}{(1-\theta)^{3}}\left[4-3 \theta+\theta^{2}-6 \alpha \theta^{2}+3 \alpha \theta^{3}+2 \alpha^{2} \theta^{4}\right] .
\end{aligned}
$$

Proof: Using the characteristic function for the unity-truncated logarithmic series distribution given by (10), we immediately have

$$
\begin{aligned}
& \left.\mu_{1}=\mu=(-i) \frac{d \psi_{x}(t)}{d t}\right]_{t=0}=\frac{\alpha \theta^{2}}{1-\theta} \\
& \left.\mu_{2}=(-i)^{2} \frac{d^{2} \psi_{x}(t)}{d t^{2}}\right]_{t=0}=\frac{\alpha \theta^{2}}{(1-\theta)^{2}}[2-\theta]
\end{aligned}
$$




$$
\left.\mu_{3}^{\prime}=(-i)^{3} \frac{d^{3} \psi_{x}(t)}{d t^{3}}\right]_{t=0}=\frac{\alpha \theta^{2}}{(1-\theta)^{3}}\left[4-3 \theta+\theta^{2}\right] .
$$

These give

$$
\begin{aligned}
\mu_{2} & =\mu_{2}^{\prime}-\mu^{2} \\
& =\frac{\alpha \theta}{(1-\theta)^{2}}\left[2-\theta-\alpha \theta^{2}\right] \\
\mu_{3} & =\mu_{3}-3 \mu_{2}^{\prime} \mu+2 \mu^{3} \\
& =\frac{\alpha \theta^{2}}{(1-\theta)^{3}}\left[4-3 \theta+\theta^{2}-6 \alpha \theta^{2}+3 \alpha \theta^{3}+2 \alpha^{2} \theta^{4}\right] .
\end{aligned}
$$

Since $\mathrm{Z}$ is the sum of $\mathrm{n}$ independent random variables, its first three moments about the mean may be obtained, following Burington and May [1, p. 37], as the sum of the moments about the mean of the $x_{j}$ 's and this completes the proof.

Property 2: The probability function $p(z ; n, \theta)$ with parameters $\mathrm{n}$ and $\theta$ satisfies the recurrence relation

(18) $\quad p(z+1 ; n, \theta)=\frac{\theta|C(z-n+1, z-2 n+1)|}{(z+1)|C(z-n, z-2 n)|} p(z ; n, \theta)$

Proof: Follows easily by considering the ratio of $p(z+1 ; n, \theta)$ and $p(z ; n, \theta)$.

Since the numbers $c(m, s)$ grow too big too soon 
with increase in their arguments, the recurrence relation (18) is not of much use. The next property provides a recurrence relation independent of numbers $C(m, s)$ and useful for tabulation purposes.

Property 3: The probability function $\mathrm{p}(\mathrm{z} ; \mathrm{n}, \theta)$ with parameters $\mathrm{n}$ and $\theta$ enjoys the recurrence relation

(19) $p(z+1 ; n, \theta)=\frac{\theta}{z+1}[\operatorname{an} \theta p(z-1 ; n-1, \theta)+z p(z ; n, \theta)]$ where $p(z ; n, \theta)=\frac{\alpha \theta^{2}}{z}$ for $n=1$ and $p(z ; n, \theta)=\frac{\alpha^{n_{\theta} 2 n}}{2^{n}}$ for $z=2 n$

Proof: From (17), wè have

$$
p(z+1 ; n, \theta)=\frac{\alpha^{n} n !}{(z+1) !}|C(z-n+1, z-2 n+1)| \theta^{z+1}
$$

which, using (5), may be written as

$$
p(z+1 ; n, \theta)=\frac{\alpha^{n} n !}{(z+1) !}[z|c(z-n, z-2 n+1)|
$$

$$
+z|C(z-n, z-2 n)|] \theta^{z+1}
$$

But $p(z-1 ; n-1, \theta)=\frac{\alpha^{n-1}(n-1) !}{(z-1) !}|C(z-n, z-2 n+1)| \theta^{z-1}$

and $p(z ; n, \theta)=\frac{\alpha^{n} n !}{z !}|c(z-n, z-2 n)| \theta^{z}$. Hence, we obtain (20) as 


$$
p(z+1 ; n, \theta)=\frac{\theta}{z+1}\left[\alpha n \theta_{p}(z-1 ; n-1, \theta)+z p(z ; n, \theta)\right] .
$$

Also, for $n=1$, we get

$$
\begin{aligned}
p(z ; 1, \theta) & =\frac{\alpha}{z !}|C(z-1, z-2)| \theta^{z} \\
& =\frac{\alpha \theta^{z}}{z},
\end{aligned}
$$

and for $z=2 n$, we have

$$
\begin{aligned}
p(2 n ; n, \theta) & =\frac{\alpha^{n} n !}{(2 n) !}|c(n, 0)| \theta^{2 n} \\
& =\frac{\alpha^{n} n !}{(2 n) !} \cdot 1 \cdot 3 \cdot 5 \ldots(2 n-1) \theta^{2 n} \\
& =\frac{\alpha^{n} \theta^{2 n}}{2^{n}},
\end{aligned}
$$

which completes the proof.

Theorem 2: Let $\mathrm{z}_{1}, \mathrm{z}_{2}, \ldots, \mathrm{z}_{\mathrm{k}}$ be $\mathrm{k}$ independent random variables having the probability function

$$
p\left(z_{j} ; n_{j}, \theta\right)=\frac{a^{n_{j}} n_{j} !}{z_{j} !}\left|c\left(z_{j}-n_{j}, z_{j}-2 n_{j}\right)\right| \theta^{z_{j}}
$$

where, for $j=1,2, \ldots, k, z_{j}=2 n_{j}, 2 n_{j}+1, \ldots, \infty$,

$$
=[-\log (1-\theta)-\theta]^{-1} \text {, and } 0<\theta<1 \text {. Let } Y=Z_{1}+Z_{2}+
$$


$\ldots+z_{k}$, then the distribution of $Y$ is given by $p\left(y ; \Sigma_{j=1}^{k}, \theta\right)$.

Proof: The characteristic function of $z_{j}$, using

(11), is

$$
\psi_{z j}(t)=\alpha^{n}{ }^{n}\left[-\log \left(1-\theta e^{i t}\right)-\theta e^{i t}\right]^{n} j .
$$

Since the $\mathrm{Z}_{j}$ 's are assumed to be independent, the characteristic function of $\mathrm{Y}$ is obtained as

$$
\begin{aligned}
\psi_{y}(t) & =\prod_{j=1}^{k} \psi_{z_{j}}(t) \\
& =\alpha^{\sum_{j=1}^{k} n_{j}}\left[-\log \left(1-\theta e^{i t}\right)-\theta e^{i t^{2}}\right]_{j=1}^{k} n_{j}
\end{aligned}
$$

which, by the uniqueness theorem for characteristic functions, establishes the proof.

Theorem 3: Let $X$ and $Y$ be two independent random variables having the distribution $p(x ; n, \theta)$ and $p(y ; m, \lambda)$ respectively. Let $Z=X+Y$ and $\beta=\theta / \lambda$. Then the conditional distribution of $X$. given $z=z$ is a generalized power series distribution with the series function

$$
g(\beta)=\Sigma_{x=2 n}^{z-2 m} h(x, z) \beta^{x}
$$


where

$$
h(x, z)=\frac{|C(x-n, x-2 n)|}{x !} \cdot \frac{|C(z-x-m, z-x-2 m)|}{(z-x) !}
$$

Proof: Using the definition of conditional distribution, we have

$$
\begin{aligned}
& P(X=x \mid X+Y=z)= \\
& \frac{\alpha^{n} n !|C(x-n, x-2 n)| \theta^{x}}{x !} \cdot \frac{\alpha^{m_{m} !|C(z-x-m, z-x-2 m)| \lambda^{z-x}}}{(z-x) !}
\end{aligned}
$$$$
\Sigma_{x=2 n}^{z-2 m} \frac{\alpha^{n} n !|c(x-n, x-2 n)| \theta^{x}}{x !} \cdot \frac{\alpha^{m} m|| c(z-x-m, z-x-2 m) \mid \lambda^{z-x}}{(z-x) !}
$$

Since $\theta=\beta \lambda$, we may express (22) as

$$
P(X=x \mid X+Y=z)=\frac{\frac{|C(x-n, x-2 n)|}{x !} \cdot \frac{|C(z-x-m, z-x-2 m)|}{(z-x) !} x}{\sum_{x=2 n}^{z-2 m} \frac{|C(x-n, x-2 n)|}{x !} \cdot \frac{|C(z-x-m, z-x-2 m)|}{(z-x) !} x}
$$

which can be easily recognized as the generalized power series distribution with the series function given by (2I).

Theorem 4: Let $X$ and $Y$ be two independent discrete random variables with their ranges containing $2 \mathrm{n}$ and $2 \mathrm{~m}$ respectively. Let $X+Y=Z$. If the conditional 
distribution of $\mathrm{X}$ given $\mathrm{Z}=\mathrm{z}$ is given to be a generalized power series distribution with the series function (21) for $0<\beta<\infty$ for every $z>2 n+2 m$, then each $X$ and $Y$ has the distribution $p(x ; n, \theta)$ and $p(y ; m, \lambda)$ respectively so that $\theta=\beta \lambda$.

Proof: Let $f(x)$ and $g(y)$ be the probability functions of $X$ and $Y$ respectively. We then have

(23)

$$
\frac{f(x) g(z-x)}{\sum_{x=2 n}^{z-2 m} f(x) g(z-x)}=
$$

$$
\frac{|C(x-n, x-2 n)|}{x !} \cdot \frac{|C(z-x-m, z-x-2 m)|}{(z-x) !} B^{x}
$$

Also,

(24)

$$
\begin{aligned}
& \frac{f(x-1) g(z-x+1)}{\sum_{x=2 n}^{z-2 m} f(x) g(z-x)}= \\
& \frac{\frac{|C(x-n-1, x-2 n-1)|}{(x-1) !} \cdot \frac{|C(z-x-m+1, z-x-2 m+1)|}{(z-x+1) !} B^{x}}{\sum_{x=2 n}^{z-2 m} \frac{|C(x-n, x-2 n)|}{x !} \cdot \frac{|C(z-x-m, z-x-2 m)|}{(z-x) !} B^{x}}
\end{aligned}
$$

Dividing (23) by (24) gives 
(25)

$$
\begin{aligned}
& \frac{f(x) g(z-x)}{f(x-1) g(z-x+1)}= \\
& \frac{(z-x+1)}{x} \cdot \frac{|c(x-n, x-2 n)||c(z-x-m, z-x-2 m)|}{|c(x-n-1, x-2 n-1)||c(z-x-m+1, z-x-2 m+1)|}
\end{aligned}
$$

Taking $z-x=2 m$ in (25), we get

$$
\frac{f(x) g(2 m)}{f(x-1) g(2 m+1)}=\frac{(2 m+1)|c(x-n, x-2 n)||c(m, 0)|}{x|c(x-n-1, x-2 n-1)||c(m+1,1)|} \beta
$$

which may be written as

$$
\frac{f(x)}{f(x-1)}=\frac{(x-1) !|c(x-n, x-2 n)|}{x !|c(x-n-1, x-2 n-1)|} \theta
$$

where $\theta=\frac{(2 m+1) g(2 m+1)|c(m, 0)|}{g(2 m)|c(m+1,1)|} \beta$

This gives

$$
f(x)=k \frac{|c(x-n, x-2 n)|}{x !} \theta^{x}
$$

where $k$ is a constant. Since $f(x)>0$ and $\sum_{x=2 n}^{\infty} f(x)=1$, we obtain $k=\alpha^{n} n !$. Hence, $f(x)=p(x ; n, \theta)$. A similar approach would show that $g(y)=p(y ; m, \lambda)$. 
CHAPTER V

TABULATION OF $p(z ; n, \theta)$

In this concluding chapter, a short table for the values of $p(z ; n, \theta)$ is presented. The computations were obtained using the Oregon State University terminal and are accurate to four decimal places. In order to construct this table, the recurrence relation (19) was used along with the corresponding initial values given in Property 3. Although the program was originally completed for $\mathrm{n}=I(I) 25$, the table here is furnished only for $\mathrm{n}=I(1) 15$ and $\theta=0.1(0.1) 0.9$. This is primarily due to the fact that, for $\mathrm{n}=16(1) 25$ and $\theta$ large, the values of $p(z ; n, \theta)$ do not approach zero quickly for large $z$, and hence the table would be quite extensive. 
TABLE

VALUES OF $\mathrm{p}(\mathrm{z} ; \mathrm{n}, \theta)$

THETA ( $\theta)$

\begin{tabular}{|c|c|c|c|c|c|c|c|c|c|}
\hline $\mathrm{n}$ & .1 & .2 & .3 & .4 & .5 & .6 & .7 & .8 & .9 \\
\hline 1 & .9327 & .8642 & .7940 & .7219 & .6472 & .5691 & .4861 & .3953 & .2888 \\
\hline & .0622 & .1152 & 1588 & .1925 & .2157 & .2276 & .2269 & .2108 & .1733 \\
\hline 1 & 047 & .0173 & .0357 & .0577 & .0809 & .1024 & .1191 & .1265 & .1169 \\
\hline 5 & .0004 & .0028 & .0086 & .0185 & .0324 & .0492 & .0667 & .0810 & .0842 \\
\hline E & .0000 & .0005 & .0021 & .0062 & .0135 & .0246 & .0389 & .05 & .0632 \\
\hline 7 & .0000 & .0001 & .0006 & .0021 & .0058 & .0126 & .0233 & .0370 & .0487 \\
\hline$\xi$ & 000 & .0000 & .0001 & .0007 & .0025 & .0066 & .0143 & .0259 & .0384 \\
\hline 9 & 000 & .0000 & .0000 & .0003 & .0011 & .0035 & .0089 & .01 & .0307 \\
\hline 10 & 000 & .0000 & .0000 & .0001 & .0005 & .0019 & .0056 & .0133 & .0249 \\
\hline 11 & .0000 & .0000 & .0000 & .0000 & .0002 & .0010 & .0036 & .00 & .0203 \\
\hline 12 & .0000 & .0000 & .0000 & .0000 & .0001 & .0006 & .0023 & .0 & .0168 \\
\hline 13 & .0000 & .0000 & .00 & .00 & .00 & .0003 & .0015 & .00 & .0139 \\
\hline 14 & 000 & .00 & .00 & .0000 & .0000 & .0002 & .0010 & .00 & .0117 \\
\hline 15 & .0000 & .0000 & .0000 & .0000 & .0000 & .0001 & .0006 & .0029 & .0098 \\
\hline 6 & .0000 & .0000 & .0000 & .0000 & .0000 & .0001 & .0004 & .0022 & .0083 \\
\hline 17 & .0000 & .0000 & .0000 & .0000 & .0000 & .0000 & .0003 & .0 & 0070 \\
\hline 18 & .0000 & .0000 & .0000 & .0000 & .0000 & .00 & .0002 & .0 & .0059 \\
\hline 19 & .0000 & .0000 & .0000 & .00 & .0000 & .00 & .0001 & .0 & .0051 \\
\hline 20 & .0000 & .0000 & .00 & .00 & .0000 & .00 & .0001 & .0 & .0043 \\
\hline 21 & .0000 & .0000 & .0000 & .0000 & .0000 & .0000 & .0001 & .00 & .0037 \\
\hline 22 & .0000 & .0000 & .0000 & .0000 & .0000 & .0000 & .0000 & .00 & .0032 \\
\hline 23 & .0000 & .0000 & .0000 & .0000 & .0000 & .0000 & .0000 & .0 & .0027 \\
\hline 24 & .0000 & .0000 & .0000 & .0000 & .0000 & .0000 & .0 & & 024 \\
\hline 25 & .0000 & .0000 & .0000 & .0000 & .0000 & .00 & .0 & & .0020 \\
\hline 26 & .0000 & .0000 & .0000 & .0000 & .0000 & .00 & .00 & & .0018 \\
\hline 27 & 000 & .0000 & .0000 & .0 & .0000 & .00 & .0000 & .0 & .0015 \\
\hline 28 &. & .0000 & .0 & .0 & .0000 & .00 & .0000 & .0 & .0013 \\
\hline 29 & 000 & .0000 & .0000 & .0000 & .0000 & .0000 & .0000 & .0 & .0012 \\
\hline 30 & .0000 & .0000 & .0000 & .0000 & .0000 & .0000 & .0000 & .00 & .0010 \\
\hline 1 & .0000 & .0000 & .0000 & .0000 & .0000 & .0000 & .00 & & .0009 \\
\hline 32 & .0000 & .0000 & .0000 & .0000 & .0000 & .0000 & .0000 & & .0008 \\
\hline 33 & .0000 & .0000 & .0000 & .0 & .0000 & .00 & .00 & .0 & 07 \\
\hline 34 & .0000 & .0000 & .0000 & .00 & .0 & .00 & 00 & & .0006 \\
\hline 35 & 10 & .0 & .0 & .00 & .00 & .00 & 00 & & .0005 \\
\hline 36 & & .0000 & .0 & .0000 & .0000 & .0000 & .0000 & & .0004 \\
\hline & .0000 & .0000 & .0000 & .0000 & .0000 & .0000 & .0000 & .0 & .0004 \\
\hline & .0000 & .0000 & .0000 & .0000 & .0000 & .00 & .0000 & & .0003 \\
\hline & .0000 & .0000 & .0000 & .00 & .00 & .00 & 00 & & .0003 \\
\hline 40 & 000 & 0000 & .0000 & .00 & .0000 & .0000 & .0 & & .0003 \\
\hline 4 & 000 & .0000 & .0 & .0000 & .00 & .0000 & .0 & & .0002 \\
\hline 4 & 1000 & .0000 & & .0000 & .0000 & .0000 & .0000 & .0000 & .0002 \\
\hline & & .0000 & .0000 & .0000 & .0000 & .0000 & .0000 & .0000 & .0002 \\
\hline 44 & .0000 & .0000 & .0000 & .0000 & .0000 & .0000 & .0000 & .0000 & .0002 \\
\hline 45 & .0000 & .0000 & .0000 & .0000 & .0000 & .0000 & .00 & & .0001 \\
\hline 46 & 00 & .0000 & .0000 & .0000 & .0000 & .0000 & .0000 & .0000 & .0001 \\
\hline
\end{tabular}


THETA $(\theta)$

\begin{tabular}{|c|c|c|c|c|c|c|c|c|c|}
\hline $\mathrm{n}$ & .1 & .2 & .3 & .4 & .5 & .6 & .7 & .8 & .9 \\
\hline 14 & 1000 & 0 & & 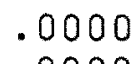 & & .0000 & .0000 & 10 & .0001 \\
\hline & & .0000 & .0000 & .0000 & .0000 & .00 & .0000 & .0000 & .0001 \\
\hline \multirow[t]{44}{*}{2} & .8700 & .7468 & .6304 & .5211 & .4188 & .3239 & .2363 & .1563 & .0834 \\
\hline & $: 1160$ & .1991 & .2522 & .2779 & .2792 & .2591 & .2206 & .1667 & .1001 \\
\hline & .0126 & .0431 & .0820 & .1204 & .1512 & .1684 & .1673 & .1445 & .0976 \\
\hline & .0013 & .0088 & .0250 & .0489 & .0768 & .1026 & .1189 & .1174 & .0891 \\
\hline & .0001 & .0017 & .0074 & .0193 & .0380 & .0609 & .0823 & .0928 & .079 \\
\hline & .0000 & .0003 & .0022 & .0076 & .0185 & .0357 & .0562 & .0725 & \\
\hline & .0000 & .0001 & .0006 & .0029 & .0090 & .0208 & .0382 & .0563 & .0609 \\
\hline & .0000 & .0000 & .0002 & .0011 & .0044 & .0121 & .0259 & .0436 & .0530 \\
\hline & .0000 & .0000 & .0001 & .0004 & .0021 & .0070 & .0175 & .0337 & .0462 \\
\hline & .0000 & .0000 & .0000 & .0002 & .0010 & .0041 & .0119 & .0261 & .0402 \\
\hline & .0000 & .0000 & .0000 & .0001 & .0005 & .0024 & .0080 & .0202 & .0349 \\
\hline & .0000 & .0000 & .0000 & .0000 & .0002 & .00 & .00 & .0156 & .030 \\
\hline & .0000 & .0000 & .0000 & .0000 & .0001 & .0008 & .0037 & .0121 & .0265 \\
\hline & .0000 & .0000 & .0000 & .0000 & .00 & .0005 & .00 & .0094 & .0231 \\
\hline & .0000 & .0000 & .0000 & .0000 & .0000 & .0003 & .0017 & .0073 & .0202 \\
\hline & .0000 & .0000 & .0000 & .0000 & .00 & .0002 & .0012 & .0056 & .0176 \\
\hline & .0000 & .0000 & .0000 & .0000 & .0000 & .0001 & .0008 & .0044 & .0154 \\
\hline & 000 & .0000 & .0000 & .0000 & .0000 & .0001 & .0005 & .0034 & .0135 \\
\hline & .0000 & .0000 & .0000 & .0000 & .0000 & .0000 & .0004 & .0027 & .01 .18 \\
\hline & .0000 & .0000 & .0000 & .0000 & .0000 & .0000 & .0002 & .0021 & .0104 \\
\hline & .0000 & .0000 & .0000 & .0000 & .0000 & .0000 & .0002 & .0016 & .009 \\
\hline & .0000 & .0000 & .0000 & .0000 & .0000 & .0000 & .0001 & .0013 & .0080 \\
\hline & .0000 & .0000 & .0000 & .0000 & .00 & & & & \\
\hline & .0000 & .0000 & .0000 & .0000 & .0000 & .0000 & .0001 & .0008 & .0062 \\
\hline & 0000 & .0000 & & & & & & & \\
\hline & .0000 & .0000 & .0000 & .0000 & .0000 & .0000 & .0000 & .0005 & .0048 \\
\hline & & & & & & & & & .00 \\
\hline & .0000 & .0000 & .0000 & .0000 & .0000 & .0000 & .0 & .0003 & .0037 \\
\hline & & & & & & & & & \\
\hline & .0000 & .0000 & .0000 & .0000 & .0000 & .0000 & .0 & .0002 & .002 \\
\hline & & & & & & & & & \\
\hline & .0000 & .0000 & .0000 & .0000 & .0000 & .0000 & .0000 & .0001 & .0022 \\
\hline & & & & & & & & & \\
\hline & .0000 & .0000 & .0000 & .0000 & .00 & .00 & .0000 & .0001 & .0018 \\
\hline & & & 00 & .00 & .00 & .00 & .05 & .00 & .00 \\
\hline & .0000 & .0000 & .0000 & .0000 & .00 & .0000 & .0000 & .0000 & .0014 \\
\hline & & & .00 & .00 & .00 & .00 & .00 & .00 & .001 \\
\hline & .0000 & .0000 & .0000 & .0000 & .0000 & .0000 & .0000 & .0000 & .0011 \\
\hline & & .0000 & .0000 & .0000 & .0000 & .0000 & .0000 & .0000 & .0010 \\
\hline & .0000 & .0000 & .0000 & .0000 & .0000 & .0000 & .0000 & .0000 & \\
\hline & & 0 & .0 & .0000 & .0000 & .0000 & .0000 & .0000 & .0007 \\
\hline & .0000 & .0000 & .0000 & .0000 & .0000 & .0000 & .0000 & .0000 & .0007 \\
\hline & .0000 & .0000 & .0000 & .0000 & .0000 & .0000 & .0000 & .0000 & .0006 \\
\hline & & & & .0000 & & .0000 & .0000 & .0000 & \\
\hline
\end{tabular}


THETA $(\theta)$

\begin{tabular}{|c|c|c|c|c|c|c|c|c|c|c|}
\hline$n$ & $z$ & .1 & .2 & .3 & .4 & .5 & .6 & .7 & .8 & .9 \\
\hline 2 & $\begin{array}{l}48 \\
49 \\
50 \\
51 \\
52 \\
53 \\
54 \\
55 \\
56 \\
57 \\
58 \\
59 \\
60 \\
61\end{array}$ & $\begin{array}{l}.0000 \\
.0000 \\
.0000 \\
.0000 \\
.0000 \\
.0000 \\
.0000 \\
.0000 \\
.0000 \\
.0000 \\
.0000 \\
.0000\end{array}$ & $\begin{array}{l}.0000 \\
.0000 \\
.0000 \\
.0000 \\
.0000 \\
.0000 \\
.0000 \\
.0000 \\
.0000 \\
.0000 \\
.0000 \\
.0000 \\
.0000\end{array}$ & $\begin{array}{l}.0000 \\
.0000 \\
.0000 \\
.0000 \\
.0000 \\
.0000 \\
.0000 \\
.0000 \\
.0000 \\
.0000 \\
.0000 \\
.0000 \\
.0000 \\
.0000\end{array}$ & $\begin{array}{l}.0000 \\
.0000 \\
.0000 \\
.0000 \\
.0000 \\
.0000 \\
.0000 \\
.0000 \\
.0000 \\
.0000 \\
.0000 \\
.0000\end{array}$ & $\begin{array}{l}.0000 \\
.0000 \\
.0000 \\
.0000 \\
.0000 \\
.0000 \\
.0000 \\
.0000 \\
.0000 \\
.0000 \\
.0000 \\
.0000 \\
.0000 \\
.0000\end{array}$ & $\begin{array}{l}.0000 \\
.0000 \\
.0000 \\
.0000 \\
.0000 \\
.0000 \\
.0000 \\
.0000 \\
.0000 \\
.0000 \\
.0000 \\
.0000 \\
.0000 \\
.0000\end{array}$ & $\begin{array}{l}.00 \\
.00 \\
.00 \\
.00 \\
.00 \\
.00 \\
.00 \\
.00 \\
.00 \\
.00 \\
.00 \\
.00\end{array}$ & $\begin{array}{l}500 \\
5000 \\
5000 \\
5000 \\
500 \\
5000 \\
500 \\
5000 \\
5000 \\
5000 \\
000 \\
000 \\
5000 \\
5000\end{array}$ & $\begin{array}{l}.0005 \\
.0004 \\
.0004 \\
.0003 \\
.0003 \\
.0002 \\
.0002 \\
.0002 \\
.0002 \\
.0002 \\
.0001 \\
.0001 \\
.0001 \\
.0001\end{array}$ \\
\hline 3 & $\begin{array}{r}6 \\
7 \\
8 \\
9 \\
10 \\
11 \\
12 \\
13 \\
14 \\
15 \\
16 \\
17 \\
18 \\
19 \\
20 \\
21 \\
22 \\
23 \\
24 \\
25 \\
26 \\
27 \\
28 \\
29 \\
30 \\
31 \\
32 \\
33 \\
34 \\
35 \\
36 \\
37\end{array}$ & $\begin{array}{l}.1623 \\
.0230 \\
.0028 \\
.0003 \\
.0000 \\
.0000 \\
.0000 \\
.0000 \\
.0000 \\
.0000 \\
.0000 \\
.0000 \\
.0000 \\
.0000 \\
.0000 \\
.0000 \\
.0000 \\
.0000 \\
.0000 \\
.0000 \\
.0000 \\
.0000 \\
.0000 \\
.0000 \\
.0000 \\
.0000 \\
.0000 \\
.0000 \\
.0000 \\
.0000 \\
.0000\end{array}$ & $\begin{array}{l}.6454 \\
.2581 \\
.0731 \\
.0181 \\
.0041 \\
.0009 \\
.0002 \\
.0000 \\
.0000 \\
.0000 \\
.0000 \\
.0000 \\
.0000 \\
.0000 \\
.0000 \\
.0000 \\
.0000 \\
.0000 \\
.0000 \\
.0000 \\
.0000 \\
.0000 \\
.0000 \\
.0000 \\
.0000 \\
.0000 \\
.0000 \\
.0000 \\
.0000 \\
.0000 \\
.0000 \\
.0000\end{array}$ & $\begin{array}{l}.5006 \\
.3003 \\
.1276 \\
.0473 \\
.0163 \\
.0054 \\
.0017 \\
.0005 \\
.0002 \\
.0001 \\
.0000 \\
.0000 \\
.0000 \\
.0000 \\
.0000 \\
.0000 \\
.0000 \\
.0000 \\
.0000 \\
.0000 \\
.0000 \\
.0000 \\
.0000 \\
.0000 \\
.0000 \\
.0000 \\
.0000 \\
.0000 \\
.0000 \\
.0000 \\
.0000 \\
.0000\end{array}$ & $\begin{array}{l}.1705 \\
.1185 \\
.0387 \\
.0170 \\
.0073 \\
.0031 \\
.0013 \\
.0005 \\
.0002 \\
.0001 \\
.0000 \\
.0000 \\
.0000 \\
.0000 \\
.0000 \\
.0000 \\
.0000 \\
.0000 \\
.0000 \\
.0000 \\
.0000 \\
.0000 \\
.0000 \\
.0000 \\
.0000 \\
.0000 \\
.0000 \\
.0000 \\
.0000 \\
.0000\end{array}$ & $\begin{array}{l}.2711 \\
.2711 \\
.1920 \\
.1302 \\
.0680 \\
.0375 \\
.0201 \\
.0106 \\
.0055 \\
.0028 \\
.0014 \\
.0007 \\
.0004 \\
.0002 \\
.0001 \\
.0000 \\
.0000 \\
.0000 \\
.0000 \\
.0000 \\
.0000 \\
.0000 \\
.0000 \\
.0000 \\
.0000 \\
.0000 \\
.0000 \\
.0000 \\
.0000 \\
.0000 \\
.0000 \\
.0000\end{array}$ & $\begin{array}{l}.1843 \\
.2212 \\
.1880 \\
.1392 \\
.0959 \\
.0634 \\
.0408 \\
.0258 \\
.0161 \\
.0099 \\
.0061 \\
.0037 \\
.0023 \\
.0014 \\
.0008 \\
.0005 \\
.0003 \\
.0002 \\
.0001 \\
.0001 \\
.0000 \\
.0000 \\
.0000 \\
.0000 \\
.0000 \\
.0000 \\
.0000 \\
.0000 \\
.0000 \\
.0000 \\
.0000 \\
.0000\end{array}$ & $\begin{array}{l}.0011 \\
.0007 \\
.0005 \\
.0004 \\
.0003 \\
.0002 \\
.0001 \\
.0001 \\
.0001 \\
.0000 \\
.0000 \\
.0000 \\
.0000 \\
.0000\end{array}$ & $\begin{array}{l}.0618 \\
.0989 \\
.1120 \\
.1106 \\
.1017 \\
.0896 \\
.0768 \\
.0647 \\
.0538 \\
.0443 \\
.0362 \\
.0294 \\
.0238 \\
.0192 \\
.0155 \\
.0124 \\
.0100 \\
.0080 \\
.0064 \\
.0051 \\
.0041 \\
.0032 \\
.0026 \\
.0021 \\
.0016 \\
.0013 \\
.0010 \\
.0008 \\
.0007 \\
.0005 \\
.0004 \\
.0003\end{array}$ & $\begin{array}{l}.0241 \\
.0433 \\
.0553 \\
.0614 \\
.0634 \\
.0629 \\
.0607 \\
.0575 \\
.0537 \\
.0498 \\
.0458 \\
.0419 \\
.0382 \\
.0346 \\
.0314 \\
.0283 \\
.0256 \\
.0230 \\
.0207 \\
.0186 \\
.0167 \\
.0150 \\
.0134 \\
.01211 \\
.0108 \\
.0097 \\
.0087 \\
.0078 \\
.0069 \\
.0062 \\
.0056 \\
.0050\end{array}$ \\
\hline
\end{tabular}


THETA $(\theta)$

\begin{tabular}{|c|c|c|c|c|c|c|c|c|c|c|}
\hline$n$ & $z$ & . & .2 & .3 & .4 & .5 & .6 & 7 & .8 & .9 \\
\hline 3 & $\begin{array}{l}38 \\
39 \\
40 \\
41 \\
42 \\
43 \\
44 \\
45 \\
46 \\
47 \\
48 \\
49 \\
50 \\
51 \\
52 \\
53 \\
54 \\
55 \\
56 \\
57 \\
58 \\
59 \\
60 \\
61 \\
62 \\
63 \\
64 \\
65 \\
66 \\
67 \\
68 \\
69 \\
70 \\
71 \\
72 \\
73\end{array}$ & $\begin{array}{l}.0000 \\
.0000 \\
.0000 \\
.0000 \\
.0000 \\
.0000 \\
.0000 \\
.0000 \\
.0000 \\
.0000 \\
.0000 \\
.0000 \\
.0000 \\
.0000 \\
.0000 \\
.0000 \\
.0000 \\
.0000 \\
.0000 \\
.0000 \\
.0000 \\
.0000 \\
.0000 \\
.0000 \\
.0000 \\
.0000 \\
.0000 \\
.0000 \\
.0000 \\
.0000 \\
.0000 \\
.0000 \\
.0000 \\
.0000 \\
.0000 \\
.0000\end{array}$ & $\begin{array}{l}.0000 \\
.0000 \\
.0000 \\
.0000 \\
.0000 \\
.0000 \\
.0000 \\
.0000 \\
.0000 \\
.0000 \\
.0000 \\
.0000 \\
.0000 \\
.0000 \\
.0000 \\
.0000 \\
.0000 \\
.0000 \\
.0000 \\
.0000 \\
.0000 \\
.0000 \\
.0000 \\
.0000 \\
.0000 \\
.0000 \\
.0000 \\
.0000 \\
.0000 \\
.0000 \\
.0000 \\
.0000 \\
.0000 \\
.0000 \\
.0000 \\
.0000\end{array}$ & $\begin{array}{l}.0000 \\
.0000 \\
.0000 \\
.0000 \\
.0000 \\
.0000 \\
.0000 \\
.0000 \\
.0000 \\
.0000 \\
.0000 \\
.0000 \\
.0000 \\
.0000 \\
.0000 \\
.0000 \\
.0000 \\
.0000 \\
.0000 \\
.0000 \\
.0000 \\
.0000 \\
.0000 \\
.0000 \\
.0000 \\
.0000 \\
.0000 \\
.0000 \\
.0000 \\
.0000 \\
.0000 \\
.0000 \\
.0000 \\
.0000 \\
.0000 \\
.0000\end{array}$ & $\begin{array}{l}.0000 \\
.0000 \\
.0000 \\
.0000 \\
.0000 \\
.0000 \\
.0000 \\
.0000 \\
.0000 \\
.0000 \\
.0000 \\
.0000 \\
.0000 \\
.0000 \\
.0000 \\
.0000 \\
.0000 \\
.0000 \\
.0000 \\
.0000 \\
.0000 \\
.0000 \\
.0000 \\
.0000 \\
.0000 \\
.0000 \\
.0000 \\
.0000 \\
.0000 \\
.0000 \\
.0000 \\
.0000 \\
.0000 \\
.0000 \\
.0000 \\
.0000\end{array}$ & $\begin{array}{l}.0000 \\
.0000 \\
.0000 \\
.0000 \\
.0000 \\
.0000 \\
.0000 \\
.0000 \\
.0000 \\
.0000 \\
.0000 \\
.0000 \\
.0000 \\
.0000 \\
.0000 \\
.0000 \\
.0000 \\
.0000 \\
.0000 \\
.0000 \\
.0000 \\
.0000 \\
.0000 \\
.0000 \\
.0000 \\
.0000 \\
.0000 \\
.0000 \\
.0000 \\
.0000 \\
.0000 \\
.0000 \\
.0000 \\
.0000 \\
.0000 \\
.0000\end{array}$ & $\begin{array}{l}.0000 \\
.0000 \\
.0000 \\
.0000 \\
.0000 \\
.0000 \\
.0000 \\
.0000 \\
.0000 \\
.0000 \\
.0000 \\
.0000 \\
.0000 \\
.0000 \\
.0000 \\
.0000 \\
.0000 \\
.0000 \\
.0000 \\
.0000 \\
.0000 \\
.0000 \\
.0000 \\
.0000 \\
.0000 \\
.0000 \\
.0000 \\
.0000 \\
.0000 \\
.0000 \\
.0000 \\
.0000 \\
.0000 \\
.0000 \\
.0000 \\
.0000\end{array}$ & $\begin{array}{l}.0000 \\
.0000 \\
.0000 \\
.0000 \\
.0000 \\
.0000 \\
.0000 \\
.0000 \\
.0000 \\
.0000 \\
.0000 \\
.0000 \\
.0000 \\
.0000 \\
.0000 \\
.0000 \\
.0000 \\
.0000 \\
.0000 \\
.0000 \\
.0000 \\
.0000 \\
.0000 \\
.0000 \\
.0000 \\
.0000 \\
.0000 \\
.0000 \\
.0000 \\
.0000 \\
.0000 \\
.0000 \\
.0000 \\
.0000 \\
.0000 \\
.0000\end{array}$ & $\begin{array}{l}.0003 \\
.0002 \\
.0002 \\
.0001 \\
.0001 \\
.0001 \\
.0001 \\
.0001 \\
.0000 \\
.0000 \\
.0000 \\
.0000 \\
.0000 \\
.0000 \\
.0000 \\
.0000 \\
.0000 \\
.0000 \\
.0000 \\
.0000 \\
.0000 \\
.0000 \\
.0000 \\
.0000 \\
.0000 \\
.0000 \\
.0000 \\
.0000 \\
.0000 \\
.0000 \\
.0000 \\
.0000 \\
.0000 \\
.0000 \\
.0000 \\
.0000\end{array}$ & $\begin{array}{l}.0044 \\
.0040 \\
.0036 \\
.0032 \\
.0028 \\
.0025 \\
.0023 \\
.0020 \\
.0018 \\
.0016 \\
.0015 \\
.0013 \\
.0012 \\
.0010 \\
.0009 \\
.0008 \\
.0007 \\
.0007 \\
.0006 \\
.0005 \\
.0005 \\
.0004 \\
.0004 \\
.0003 \\
.0003 \\
.0003 \\
.0002 \\
.0002 \\
.0002 \\
.0002 \\
.0001 \\
.0001 \\
.0001 \\
.0001 \\
.0001 \\
.0000\end{array}$ \\
\hline 4 & $\begin{array}{r}8 \\
9 \\
10 \\
11 \\
12 \\
13 \\
14 \\
15 \\
16 \\
17\end{array}$ & $\begin{array}{l}.7569 \\
.2018 \\
.0353 \\
.0051 \\
.0007 \\
.0001 \\
.0000 \\
.0000 \\
.0000 \\
.0000\end{array}$ & $\begin{array}{l}.5577 \\
.2074 \\
.1041 \\
.0303 \\
.0079 \\
.0020 \\
.0005 \\
.0001 \\
.0000 \\
.0000\end{array}$ & $\begin{array}{l}.3180 \\
.1669 \\
.0728 \\
.0286 \\
.0106 \\
.0037 \\
.0013 \\
.0004 \\
.0001\end{array}$ & $\begin{array}{l}.2896 \\
.2027 \\
.1179 \\
.0618 \\
.0304 \\
.0143 \\
.0065 \\
.0029 \\
.0013\end{array}$ & $\begin{array}{l}.1754 \\
.2339 \\
.2047 \\
.1488 \\
.0976 \\
.0599 \\
.0353 \\
.0201 \\
.0112 \\
.0061\end{array}$ & $\begin{array}{l}.1049 \\
.1678 \\
.1762 \\
.1537 \\
.1210 \\
.0892 \\
.0630 \\
.0431 \\
.0288 \\
.0189\end{array}$ & $\begin{array}{l}.0559 \\
.1043 \\
.1277 \\
.1300 \\
.1193 \\
.1027 \\
.0845 \\
.0675 \\
.0526 \\
.0403\end{array}$ & $\begin{array}{l}.0244 \\
.0521 \\
.0730 \\
.0849 \\
.0890 \\
.0875 \\
.0824 \\
.0751 \\
.0670 \\
.0587\end{array}$ & $\begin{array}{l}.0070 \\
.0167 \\
.0263 \\
.0344 \\
.0406 \\
.0449 \\
.0475 \\
.0488 \\
.0489 \\
.0482\end{array}$ \\
\hline
\end{tabular}


THETA $(\theta)$

\begin{tabular}{|c|c|c|c|c|c|c|c|c|c|c|}
\hline $\mathrm{n}$ & $\mathbf{z}$ & .1 & .2 & .3 & .4 & .5 & .6 & .7 & .8 & .9 \\
\hline 4 & 18 & 0000 & .0000 & .0000 & .0006 & .0033 & 3 & 15 & 7 & .0468 \\
\hline & 19 & .0000 & .0000 & & & 18 & & .0228 & .0433 & .0450 \\
\hline & 20 & .0000 & .0000 & .0000 & .0001 & .0009 & .0050 & .0169 & .0367 & .0429 \\
\hline & 21 & .0000 & .0000 & .0000 & .0000 & .0005 & .0031 & .0124 & .0308 & .0406 \\
\hline & 22 & .0000 & .0000 & .0000 & .0000 & .0003 & .0020 & .0091 & .0258 & .0382 \\
\hline & 23 & .0000 & .0000 & .0000 & .0000 & .0001 & .0012 & $.006 \overline{6}$ & .0214 & .0357 \\
\hline & 24 & .0000 & .0000 & 00 & .0000 & .0001 & .0008 & .0048 & .0177 & .0333 \\
\hline & 25 & .0000 & .0000 & .0000 & .0000 & .0000 & .0005 & .0035 & .01 & .0309 \\
\hline & 26 & .0000 & .0000 & .0000 & .0000 & .0000 & .0003 & .0025 & .01 & .0285 \\
\hline & 27 & .0000 & .0000 & .0000 & .0000 & .0000 & .0002 & .0018 & .00 & .0263 \\
\hline & 28 & .0000 & .0000 & .0000 & .0000 & .0000 & .0001 & .0013 & .00 & .0242 \\
\hline & 29 & .0000 & .0000 & .0000 & .0000 & .0000 & .0001 & .00 & .00 & .0222 \\
\hline & 30 & .0000 & .0000 & .0000 & .00 & .0000 & .00 & .00 & .0 & .0204 \\
\hline & 31 & .0000 & .0000 & .0 & .00 & .0000 & .00 & .00 & .00 & .0187 \\
\hline & 32 & .0000 & .0000 & 00 & .0000 & .0000 & .0000 & .0003 & .00 & .0170 \\
\hline & 33 & .0000 & .0000 & .0000 & .0000 & .0000 & .0000 & .0002 & .00 & .0156 \\
\hline & 34 & .0000 & .0000 & .0000 & .0000 & .0000 & .00 & .00 & .0 & .0142 \\
\hline & 35 & .0000 & .0000 & .0000 & .0000 & .0000 & .00 & .00 & & .0129 \\
\hline & 36 & .0000 & .0000 & .0000 & .0000 & .0000 & .00 & .0 & .0 & .0117 \\
\hline & 37 & .0000 & .0000 & .0 & .00 & .0000 & .00 & .00 & & .0107 \\
\hline & 38 & .0000 & .0000 & .0 & .0 & .0 & .00 & .0 & & .0097 \\
\hline & 39 & .00 & .00 & .0 & .0000 & .0000 & .00 & .0000 & .00 & .0088 \\
\hline & 40 & .0000 & .0000 & .0000 & .0000 & .0000 & .0000 & .0000 & .00 & .0080 \\
\hline & 41 & .0000 & .0000 & .0000 & .0000 & .0000 & .0000 & .00 & .00 & .0072 \\
\hline & 42 & .0000 & .0000 & .0000 & .0000 & .0 & .0 & & & 65 \\
\hline & 43 & .0000 & .0000 & .0 & .0 & & .0 & .0 & .0 & 59 \\
\hline & 44 & .0000 & .0000 & .0 & .0 & 0 & .0 &. & .0 & .0 \\
\hline & 45 & .0000 & .0000 & .0 & .0 & .0000 & .00 & .0 & .0 & .0048 \\
\hline & 46 & .00 & .0 & .0 & .0 & .0000 & .00 & .0 & .0 & .0044 \\
\hline & 47 & .0 & .0000 & .0 & .00 & .0000 & .00 & .0 & & .0040 \\
\hline & 48 & .0000 & .0000 & .00 & .0000 & .0000 & .00 & .0 & .0 & .0036 \\
\hline & 49 & .0000 & .0000 & .0000 & .0000 & .0000 & .00 & .0 & & .0032 \\
\hline & 50 & .0000 & .0000 & .00 & .0 & .0000 & .00 & & & 29 \\
\hline & 51 & .0000 & .0000 & & .0 & & & & & 26 \\
\hline & 52 & & .0000 & & .0 & .0 & .00 & .0 & .0 & .0024 \\
\hline & 53 & .0 & .0000 & .0 & .0000 & .0000 & .0000 & .0000 & .00 & .0021 \\
\hline & 51 & .0000 & .0000 & .0000 & .0000 & .0000 & .0000 & .00 & .00 & .0019 \\
\hline & 55 & .0000 & .0000 & .0000 & .0000 & .0000 & .00 & .00 & .0 & .0017 \\
\hline & 56 & .0000 & .0000 & .0000 & .0000 & .0000 & .00 & .0 & .0 & .0016 \\
\hline & & .0000 & .0000 & .0 & .01 & .0000 & .00 & & & .0014 \\
\hline & & .0000 & .0000 & & .0 & & .00 & & .0 & .0013 \\
\hline & & .0000 & .0000 & & .0000 & & .00 & & & \\
\hline & & & .0000 & .0000 & .0000 & .0000 & .0000 & .0000 & .0000 & .0010 \\
\hline & 6 & .0000 & .0000 & .0000 & .0000 & .0000 & .0000 & .0000 & .0000 & .0009 \\
\hline & 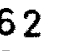 & .0000 & .0000 & & .0000 & .0000 & .00 & & .0 & .0008 \\
\hline & & .0000 & .0000 & & & & .00 & & & .0008 \\
\hline & & .0000 & .0000 & 0 & .0000 & .0000 & .0000 & .0000 & .0000 & .0007 \\
\hline
\end{tabular}


THETA ( $\theta)$

\begin{tabular}{|c|c|c|c|c|c|c|c|c|c|c|}
\hline $\mathrm{n}$ & $\mathbf{z}$ & .1 & .2 & .3 & .4 & .5 & .6 & .7 & .8 & .9 \\
\hline 4 & $\begin{array}{l}65 \\
66 \\
67 \\
68 \\
69 \\
70 \\
71 \\
72 \\
73 \\
74 \\
75 \\
76 \\
77 \\
78 \\
79 \\
80 \\
81 \\
82\end{array}$ & $\begin{array}{l}.0000 \\
.0000 \\
.0000 \\
.0000 \\
.0000 \\
.0000 \\
.0000 \\
.0000 \\
.0000 \\
.0000 \\
.0000 \\
.0000 \\
.0000 \\
.0000 \\
.0000 \\
.0000 \\
.0000\end{array}$ & $\begin{array}{l}.0000 \\
.0000 \\
.0000 \\
.0000 \\
.0000 \\
.0000 \\
.0000 \\
.0000 \\
.0000 \\
.0000 \\
.0000 \\
.0000 \\
.0000 \\
.0000 \\
.0000 \\
.0000 \\
.0000 \\
.0000\end{array}$ & $\begin{array}{l}.0000 \\
.0000 \\
.0000 \\
.0000 \\
.0000 \\
.0000 \\
.0000 \\
.0000 \\
.0000 \\
.0000 \\
.0000 \\
.0000 \\
.0000 \\
.0000 \\
.0000 \\
.0000 \\
.0000 \\
.0000\end{array}$ & $\begin{array}{l}.0000 \\
.0000 \\
.0000 \\
.0000 \\
.0000 \\
.0000 \\
.0000 \\
.0000 \\
.0000 \\
.0000 \\
.0000 \\
.0000 \\
.0000 \\
.0000 \\
.0000 \\
.0000\end{array}$ & $\begin{array}{l}.0000 \\
.0000 \\
.0000 \\
.0000 \\
.0000 \\
.0000 \\
.0000 \\
.0000 \\
.0000 \\
.0000 \\
.0000 \\
.0000 \\
.0000 \\
.0000 \\
.0000 \\
.0000 \\
.0000 \\
.0000\end{array}$ & $\begin{array}{l}.0000 \\
.0000 \\
.0000 \\
.0000 \\
.0000 \\
.0000 \\
.0000 \\
.0000 \\
.0000 \\
.0000 \\
.0000 \\
.0000 \\
.0000 \\
.0000 \\
.0000 \\
.0000 \\
.0000 \\
.0000\end{array}$ & $\begin{array}{l}.0000 \\
.0000 \\
.0000 \\
.0000 \\
.0000 \\
.0000 \\
.0000 \\
.0000 \\
.0000 \\
.0000 \\
.0000 \\
.0000 \\
.0000 \\
.0000 \\
.0000 \\
.0000 \\
.0000 \\
.0000\end{array}$ & $\begin{array}{l}.0000 \\
.0000 \\
.0000 \\
.0000 \\
.0000 \\
.0000 \\
.0000 \\
.0000 \\
.0000 \\
.0000 \\
.0000 \\
.0000 \\
.0000 \\
.0000 \\
.0000 \\
.0000 \\
.0000 \\
.0000\end{array}$ & $\begin{array}{l}.0006 \\
.0006 \\
.0005 \\
.0004 \\
.0004 \\
.0004 \\
.0003 \\
.0003 \\
.0003 \\
.0002 \\
.0002 \\
.0002 \\
.0002 \\
.0001 \\
.0001 \\
.0001 \\
.0001 \\
.0001\end{array}$ \\
\hline 5 & $\begin{array}{l}10 \\
11 \\
12 \\
13 \\
14 \\
15 \\
16 \\
17 \\
18 \\
19 \\
20 \\
21 \\
22 \\
23 \\
24 \\
25 \\
26 \\
27 \\
28 \\
29 \\
30 \\
31 \\
32 \\
33 \\
34 \\
35 \\
36 \\
37\end{array}$ & $\begin{array}{l}.0490 \\
.0082 \\
.0012 \\
.0002 \\
.0000 \\
.0000 \\
.0000 \\
.0000 \\
.0000 \\
.0000 \\
.0000 \\
.0000 \\
.0000 \\
.0000 \\
.0000 \\
.0000 \\
.0000 \\
.0000 \\
.0000 \\
.0000 \\
.0000 \\
.0000 \\
.0000 \\
.0000 \\
.0000 \\
.0000\end{array}$ & $\begin{array}{l}.1339 \\
.0448 \\
.0132 \\
.0036 \\
.0009 \\
.0002 \\
.0001 \\
.0000 \\
.0000 \\
.0000 \\
.0000 \\
.0000 \\
.0000 \\
.0000 \\
.0000 \\
.0000 \\
.0000 \\
.0000 \\
.0000 \\
.0000 \\
.0000 \\
.0000 \\
.0000 \\
.0000 \\
.0000 \\
.0000\end{array}$ & $\begin{array}{l}.3156 \\
.3156 \\
.1972 \\
.0991 \\
.0438 \\
.0179 \\
.0069 \\
.0025 \\
.0009 \\
.0003 \\
.0001 \\
.0000 \\
.0000 \\
.0000 \\
.0000 \\
.0000 \\
.0000 \\
.0000 \\
.0000 \\
.0000 \\
.0000 \\
.0000 \\
.0000 \\
.0000 \\
.0000 \\
.0000 \\
.0000 \\
.0000\end{array}$ & $\begin{array}{l}.2178 \\
.1450 \\
.0861 \\
.0468 \\
.0240 \\
.0118 \\
.0056 \\
.0026 \\
.0012 \\
.0005 \\
.0002 \\
.0001 \\
.0000 \\
.0000 \\
.0000 \\
.0000 \\
.0000 \\
.0000 \\
.0000 \\
.0000 \\
.0000 \\
.0000 \\
.0000 \\
.0000 \\
.0000 \\
.0000\end{array}$ & $\begin{array}{l}.1135 \\
.1892 \\
.1971 \\
.0650 \\
.1217 \\
.0827 \\
.0530 \\
.0326 \\
.0194 \\
.0113 \\
.0064 \\
.0036 \\
.0020 \\
.0011 \\
.0006 \\
.0003 \\
.0002 \\
.0001 \\
.0000 \\
.0000 \\
.0000 \\
.0000 \\
.0000 \\
.0000 \\
.0000 \\
.0000 \\
.0000 \\
.0000\end{array}$ & $\begin{array}{l}.0597 \\
.1194 \\
.1492 \\
.1500 \\
.1327 \\
.1082 \\
.0832 \\
.0614 \\
.0439 \\
.0306 \\
.0209 \\
.0141 \\
.0094 \\
.0062 \\
.0040 \\
.0026 \\
.0017 \\
.0011 \\
.0007 \\
.0004 \\
.0003 \\
.0002 \\
.0001 \\
.0001 \\
.0000 \\
.0000 \\
.0000 \\
.0000\end{array}$ & $\begin{array}{l}.0272 \\
.0634 \\
.0924 \\
.1083 \\
.1118 \\
.1063 \\
.0955 \\
.0822 \\
.0685 \\
.0558 \\
.0445 \\
.0349 \\
.0271 \\
.0208 \\
.0158 \\
.0119 \\
.0089 \\
.0066 \\
.0049 \\
.0036 \\
.0026 \\
.0019 \\
.0014 \\
.0010 \\
.0007 \\
.0005 \\
.0004 \\
.0003\end{array}$ & $\begin{array}{l}.0679 \\
.0737 \\
.0757 \\
.0744 \\
.0710 \\
.0660 \\
.0602 \\
.0540 \\
.0478 \\
.0419 \\
.0364 \\
.0313 \\
.0268 \\
.0228 \\
.0193 \\
.0162 \\
.0136 \\
.0114 \\
.0095 \\
.0079 \\
.0065 \\
.0054 \\
.0045 \\
.0037\end{array}$ & $\begin{array}{l}.0020 \\
.0060 \\
.0113 \\
.0170 \\
.0226 \\
.0276 \\
.0319 \\
.0353 \\
.0378 \\
.0396 \\
.0406 \\
.0410 \\
.0409 \\
.0403 \\
.0393 \\
.0381 \\
.0367 \\
.0351 \\
.0334 \\
.0316 \\
.0299 \\
.0281 \\
.0263 \\
.0246 \\
.0229 \\
.0213 \\
.0198 \\
.0184\end{array}$ \\
\hline
\end{tabular}


THETA $(\theta)$

\begin{tabular}{|c|c|c|c|c|c|c|c|c|c|c|}
\hline$n$ & $z$ & .1 & .2 & .3 & .4 & 5 & .6 & .7 & .8 & .9 \\
\hline 5 & 38 & .0000 & .0 & 10 & .0000 & .00 & .0000 & .0002 & .0030 & .0170 \\
\hline & 39 & & & 0000 & .0000 & .00 & .00 & .0001 & .00 & 7 \\
\hline & 40 & .0000 & .0000 & .0000 & .0000 & .0000 & .0000 & .0001 & .0020 & \\
\hline & 41 & .0000 & .0000 & .0000 & .0000 & .0000 & .0000 & .0001 & .0017 & .0133 \\
\hline & 42 & & .0000 & .0000 & .0000 & & .0000 & .0001 & .0014 & 0122 \\
\hline & 43 & .0000 & .0000 & .0000 & .00 & .00 & 0 & .0000 & .0011 & .0112 \\
\hline & 44 & .0000 & .0000 & .0000 & .0000 & .0000 & .0000 & .0000 & .0009 & .0103 \\
\hline & 45 & 000 & .0000 & .0000 & .0000 & .0000 & .00 & .00 & 7 & .0095 \\
\hline & 46 & .0000 & .0000 & .0000 & .0000 & .0000 & .0000 & .00 & 6 & .0087 \\
\hline & 47 & .0000 & .0000 & .0000 & .0000 & .0000 & .0000 & .00 & .0005 & .0079 \\
\hline & 48 & 000 & .0000 & .0000 & .0000 & .00 & .0000 & .0000 & .0004 & .0073 \\
\hline & 49 & 000 & .0000 & .0000 & .0000 & .0000 & .0000 & .0000 & .0003 & .0066 \\
\hline & 50 & 000 & .0000 & .0000 & .0000 & .0000 & .0000 & .00 & .00 & .0061 \\
\hline & 51 & 000 & .0000 & .0000 & .0000 & .00 & .0 & .00 & & \\
\hline & 52 & .0000 & .0000 & .0000 & .00 & .00 & & & .0 & .0050 \\
\hline & 53 & 300 & .0000 & 0 & .00 & .00 & & & .00 & .0046 \\
\hline & 54 & 0 & .0000 & 0 & .0000 & .00 & .00 & .00 & .0001 & .0042 \\
\hline & 55 & 000 & .0000 & .0000 & .0000 & .00 & .0000 & .0000 & .00 & 0038 \\
\hline & 56 & 000 & .0000 & .0000 & .0000 & .00 & .00 & .00 & 1 & .0035 \\
\hline & 57 & 000 & .0000 & .0000 & .0000 & .00 & & & & .0032 \\
\hline & 58 & 00 & .0000 & & .00 & .00 & & & & .0 \\
\hline & 59 & & .0000 & .0 & .0 & .0 & & & .00 & .0026 \\
\hline & 60 & & .0 & 0 & .00 & .00 & & .00 & .00 & .0024 \\
\hline & 61 & 0 & .0000 & 0 & .00 & .00 & & .00 & .00 & .0021 \\
\hline & 62 & 100 & .0000 & .0000 & .0000 & .00 & .0 & .0 & .0 & .0020 \\
\hline & 63 & 000 & .0000 & .0000 & .0000 & .00 & & $\cdot$ & 0 & 18 \\
\hline & 64 & 000 & .0000 & 00 & .00 & .0 & & & & 16 \\
\hline & 65 & 000 & .0000 & & .0 & .00 & & & & .0015 \\
\hline & 6 & & .0 & & .0 & .0 & & & .0 & .0013 \\
\hline & 6 & & .0 & 10 & .00 & .00 & & .00 & .00 & .0012 \\
\hline & 68 & & .0000 & .0 & .00 & .00 & & .00 & & .0011 \\
\hline & & 0 & .0000 & .0 & .00 & .00 & & & .0 & .0010 \\
\hline & 70 & 000 & .0000 & .0000 & .00 & .00 & & & 0 & 109 \\
\hline & 71 & 100 & .0000 & & .0 & & & & & 08 \\
\hline & 72 & & .00 & & & .0 & & & & 07 \\
\hline & $n+2+$ & & & & & & & & & .0007 \\
\hline & 7 & & .0 & .0 & .0 & .00 & & & & .0006 \\
\hline & $7 !$ & 100 & .0000 & .0 & .00 & .00 & & .0 & .0 & .0005 \\
\hline & 76 & 000 & .0000 & .0000 & .00 & .00 & & .0 & .00 & .0005 \\
\hline & 77 & 000 & .0000 & .0000 & .00 & .00 & & & & .0004 \\
\hline & 78 & & .0000 & & & & & & & .0004 \\
\hline & 79 & & .00 & .0 & .00 & .00 & .0 & .00 & .0000 & $: 0004$ \\
\hline & 80 & .0 & & .0000 & & .00 & .0000 & & & 0003 \\
\hline & 81 & & & & & & & & & \\
\hline & 82 & & & .0000 & .0000 & .0000 & .0000 & .00 & .0000 & .0003 \\
\hline & 83 & & .0000 & .0000 & .0000 & .000 & & & .00 & .0002 \\
\hline & 84 & 0000 & .0000 & .0000 & .0000 & .0000 & & .0000 & .0000 & .0002 \\
\hline
\end{tabular}


THETA $(\theta)$

\begin{tabular}{|c|c|c|c|c|c|c|c|c|c|c|}
\hline $\mathrm{n}$ & z & .1 & .2 & .3 & .4 & .5 & .6 & 7 & 8 & 9 \\
\hline 5 & $\begin{array}{l}85 \\
86 \\
87 \\
88 \\
89 \\
90 \\
91\end{array}$ & $\begin{array}{l}.0000 \\
.0000 \\
.0000 \\
.0000 \\
.0000 \\
.0000 \\
.0000\end{array}$ & $\begin{array}{l}.0000 \\
.0000 \\
.0000 \\
.0000 \\
.0000 \\
.0000 \\
.0000\end{array}$ & $\begin{array}{l}.0000 \\
.0000 \\
.0000 \\
.0000 \\
.0000 \\
.0000 \\
.0000\end{array}$ & $\begin{array}{l}.0000 \\
.0000 \\
.0000 \\
.0000 \\
.0000 \\
.0000 \\
.0000\end{array}$ & $\begin{array}{l}.0000 \\
.0000 \\
.0000 \\
.0000 \\
.0000 \\
.0000 \\
.0000\end{array}$ & $\begin{array}{l}.0000 \\
.0000 \\
.0000 \\
.0000 \\
.0000 \\
.0000 \\
.0000\end{array}$ & $\begin{array}{l}.0000 \\
.0000 \\
.0000 \\
.0000 \\
.0000 \\
.0000 \\
.0000\end{array}$ & $\begin{array}{l}.0000 \\
.0000 \\
.0000 \\
.0000 \\
.0000 \\
.0000 \\
.0000\end{array}$ & $\begin{array}{l}.0002 \\
.0002 \\
.0002 \\
.0001 \\
.0001 \\
.0001 \\
.0001\end{array}$ \\
\hline 6 & $\begin{array}{l}12 \\
13 \\
14 \\
15 \\
16 \\
17 \\
18 \\
19 \\
20 \\
21 \\
22 \\
23 \\
24 \\
25 \\
26 \\
27 \\
28 \\
29 \\
30 \\
31 \\
32 \\
33 \\
34 \\
35 \\
36 \\
37 \\
38 \\
39 \\
40 \\
41 \\
42 \\
43 \\
44 \\
45 \\
46 \\
47 \\
48 \\
49 \\
50\end{array}$ & $\begin{array}{l}.0000 \\
.0000 \\
.0000 \\
.0000 \\
.0000 \\
.0000 \\
.0000 \\
.0000 \\
.0000 \\
.0000 \\
.0000 \\
.0000 \\
.0000 \\
.0000 \\
.0000 \\
.0000 \\
.0000 \\
.0000 \\
.0000 \\
.0000 \\
.0000 \\
.0000\end{array}$ & $\begin{array}{l}.4165 \\
.3332 \\
.1610 \\
.0611 \\
.0200 \\
.0060 \\
.0017 \\
.0004 \\
.0001 \\
.0000 \\
.0000 \\
.0000 \\
.0000 \\
.0000 \\
.0000 \\
.0000 \\
.0000 \\
.0000 \\
.0000 \\
.0000 \\
.0000 \\
.0000 \\
.0000 \\
.0000 \\
.0000 \\
.0000 \\
.0000 \\
.0000 \\
.0000 \\
.0000 \\
.0000 \\
.0000 \\
.0000 \\
.0000 \\
.0000 \\
.0000 \\
.0000 \\
.0000 \\
.0000\end{array}$ & $\begin{array}{l}.2506 \\
.3007 \\
.2180 \\
.1240 \\
.0610 \\
.0272 \\
.0114 \\
.0045 \\
.0017 \\
.0006 \\
.0002 \\
.0001 \\
.0000 \\
.0000 \\
.0000 \\
.0000 \\
.0000 \\
.0000 \\
.0000 \\
.0000 \\
.0000 \\
.0000 \\
.0000 \\
.0000 \\
.0000 \\
.0000 \\
.0000 \\
.0000 \\
.0000 \\
.0000 \\
.0000 \\
.0000 \\
.0000 \\
.0000 \\
.0000 \\
.0000 \\
.0000 \\
.0000 \\
.0000\end{array}$ & $\begin{array}{l}.1415 \\
.2264 \\
.2188 \\
.1659 \\
.1088 \\
.0648 \\
.0360 \\
.0190 \\
.0097 \\
.0048 \\
.0023 \\
.0011 \\
.0005 \\
.0002 \\
.0001 \\
.0000 \\
.0000 \\
.0000 \\
.0000 \\
.0000 \\
.0000 \\
.0000 \\
.0000 \\
.0000 \\
.0000 \\
.0000 \\
.0000 \\
.0000 \\
.0000 \\
.0000 \\
.0000 \\
.0000 \\
.0000 \\
.0000 \\
.0000 \\
.0000 \\
.0000 \\
.0000 \\
.0000\end{array}$ & $\begin{array}{l}.0735 \\
.1469 \\
.1776 \\
.1683 \\
.1380 \\
.1027 \\
.0714 \\
.0471 \\
.0299 \\
.0184 \\
.0111 \\
.0065 \\
.0038 \\
.0021 \\
.0012 \\
.0007 \\
.0004 \\
.0002 \\
.0001 \\
.0001 \\
.0000 \\
.0000 \\
.0000 \\
.0000 \\
.0000 \\
.0000 \\
.0000 \\
.0000 \\
.0000 \\
.0000 \\
.0000 \\
.0000 \\
.0000 \\
.0000 \\
.0000 \\
.0000 \\
.0000 \\
.0000 \\
.0000\end{array}$ & $\begin{array}{l}.0340 \\
.0815 \\
.1182 \\
.1345 \\
.1323 \\
.1182 \\
.0985 \\
.0781 \\
.0595 \\
.0440 \\
.0317 \\
.0224 \\
.0155 \\
.0106 \\
.0072 \\
.0048 \\
.0032 \\
.0021 \\
.0014 \\
.0009 \\
.0006 \\
.0004 \\
.0002 \\
.0001 \\
.0001 \\
.0001 \\
.0000 \\
.0000 \\
.0000 \\
.0000 \\
.0000 \\
.0000 \\
.0000 \\
.0000 \\
.0000 \\
.0000 \\
.0000 \\
.0000 \\
.0000\end{array}$ & $\begin{array}{l}.0132 \\
.0370 \\
.0625 \\
.0830 \\
.0952 \\
.0992 \\
.0965 \\
.0893 \\
.0793 \\
.0684 \\
.0575 \\
.0474 \\
.0384 \\
.0306 \\
.0241 \\
.0188 \\
.0146 \\
.0112 \\
.0085 \\
.0064 \\
.0049 \\
.0036 \\
.0027 \\
.0020 \\
.0015 \\
.0011 \\
.0008 \\
.0006 \\
.0004 \\
.0003 \\
.0002 \\
.0002 \\
.0001 \\
.0001 \\
.0001 \\
.0000 \\
.0000 \\
.0000 \\
.0000\end{array}$ & $\begin{array}{l}.0038 \\
.0122 \\
.0236 \\
.0358 \\
.0470 \\
.0560 \\
.0622 \\
.0657 \\
.0668 \\
.0658 \\
.0632 \\
.0595 \\
.0551 \\
.0503 \\
.0453 \\
.0404 \\
.0357 \\
.0313 \\
.0273 \\
.0236 \\
.0203 \\
.0174 \\
.0148 \\
.0126 \\
.0106 \\
.0090 \\
.0076 \\
.0063 \\
.0053 \\
.0044 \\
.0037 \\
.0031 \\
.0025 \\
.0021 \\
.0017 \\
.0014 \\
.0012 \\
.0010 \\
.0008\end{array}$ & $\begin{array}{l}.0006 \\
.0023 \\
.0045 \\
.0077 \\
.0114 \\
.0153 \\
.0191 \\
.0228 \\
.0260 \\
.0288 \\
.0312 \\
.0330 \\
.0334 \\
.0353 \\
.0358 \\
.0359 \\
.0357 \\
.0351 \\
.0345 \\
.0336 \\
.0325 \\
.0313 \\
.0300 \\
.0287 \\
.0273 \\
.0259 \\
.0245 \\
.0231 \\
.0218 \\
.0204 \\
.0191 \\
.0179 \\
.0167 \\
.0156 \\
.0145 \\
.0135 \\
.0125 \\
.0116 \\
.0107\end{array}$ \\
\hline
\end{tabular}


THETA $(\theta)$

\begin{tabular}{|c|c|c|c|c|c|c|c|c|c|c|}
\hline $\mathrm{n}$ & $z$ & .1 & .2 & .3 & .4 & .5 & .6 & .7 & .8 & .9 \\
\hline \multirow[t]{47}{*}{6} & 51 & 000 & 00 & 0000 & .0000 & & & .0000 & 0007 & .005 \\
\hline & 52 & 000 & .0000 & .0000 & .0000 & .0000 & .0000 & .0000 & .0005 & .0091 \\
\hline & 53 & 1000 & .0000 & .0000 & .0000 & .0000 & .0000 & .0000 & .0004 & 4 \\
\hline & 54 & 1000 & .0000 & .0000 & .0000 & .0000 & .0000 & .0000 & .0004 & .0078 \\
\hline & 5.5 & .0000 & .0000 & .0000 & .0000 & .0000 & .0000 & .0000 & .0003 & .0072 \\
\hline & 56 & .0000 & .0000 & .0000 & .0000 & .0000 & .0000 & .0000 & .0002 & .0066 \\
\hline & 57 & .0000 & .0000 & .0000 & .0000 & .0000 & .0000 & .0000 & .0002 & .0061 \\
\hline & 58 & .0000 & .0000 & .0000 & .0000 & .0000 & .0000 & .0000 & .0002 & 056 \\
\hline & 59 & .0000 & .0000 & .0000 & .0000 & .0000 & .0000 & .0000 & .0001 & .0051 \\
\hline & 60 & .0000 & .0000 & .0000 & .0000 & .0000 & .0000 & .0000 & .0001 & .0047 \\
\hline & 61 & .0000 & .0000 & .0000 & .0000 & .0000 & .0000 & .0000 & .0000 & .0043 \\
\hline & 62 & .0000 & .0000 & .0000 & .0000 & .0000 & .0000 & .0000 & .0000 & .0039 \\
\hline & 63 & .0000 & .0000 & .0000 & .0000 & .0000 & .0000 & .0000 & .0000 & .0036 \\
\hline & 64 & .0000 & .0000 & .0000 & .0000 & .0000 & .0000 & .0000 & .0000 & .0033 \\
\hline & 65 & .0000 & .0000 & .0000 & .0000 & .0000 & .0000 & .0000 & .0000 & .0030 \\
\hline & 66 & .0000 & .0000 & .0000 & .0000 & .0000 & .0000 & .0000 & .0000 & .0028 \\
\hline & 67 & .0000 & .0000 & .0000 & .0000 & .0000 & .0000 & .0000 & .0000 & .0025 \\
\hline & 68 & .0000 & .0000 & .0000 & .0000 & .0000 & .0000 & .0000 & .0000 & .0023 \\
\hline & 69 & .0000 & .0000 & .0000 & .0000 & .0000 & .0000 & .0000 & .0000 & .0021 \\
\hline & 70 & .0000 & .0000 & .0000 & .0000 & .0000 & .0000 & .0000 & .0000 & .0019 \\
\hline & 71 & .0000 & .0000 & .0000 & .0000 & .0000 & .00 & .0000 & .00 & .0018 \\
\hline & 72 & .0000 & .0000 & .0000 & .0000 & .0000 & .0000 & .0000 & .0000 & .0016 \\
\hline & 73 & .0000 & .0000 & .0000 & .0000 & .0000 & .00 & .0000 & .0 & .0015 \\
\hline & 74 & .0000 & .0000 & .0000 & .0000 & .0000 & .0000 & .0000 & .0000 & .0013 \\
\hline & 75 & 00 & .0000 & & & & .00 & .00 & & .0012 \\
\hline & 76 & .0000 & .0000 & .0000 & .0000 & .0000 & .0000 & .0000 & .0000 & .0011 \\
\hline & 77 & & .0000 & .00 & .00 & & .00 & .00 & & \\
\hline & 78 & .0000 & .0000 & .0000 & .0000 & .0000 & .0000 & .0000 & .0000 & .0009 \\
\hline & 79 & & .00 & & .00 & & .0000 & .0000 & .0000 & .0008 \\
\hline & 80 & .0000 & .0000 & .0000 & .0000 & .0000 & .0000 & .0000 & .0000 & .0008 \\
\hline & 81 & .0000 & .0000 & .0000 & .0000 & .0000 & .0000 & .0000 & .0000 & .0007 \\
\hline & 82 & .0000 & .0000 & .0000 & .0000 & .00 & & & & \\
\hline & 83 & .0000 & .0000 & .0000 & .0000 & .0000 & .0000 & .0000 & .0000 & .0006 \\
\hline & 84 & .0000 & .0000 & .0 & .00 & & & & & \\
\hline & 85 & .0000 & .0000 & .0000 & .0000 & .0000 & .0000 & .0000 & .0000 & .0005 \\
\hline & 86 & & & & .00 & .0 & .0 & .0 & .0 & 0 \\
\hline & 87 & .0000 & .0000 & .0 & .0000 & .0000 & .0000 & .0000 & .0000 & .0004 \\
\hline & 8 & & & & .00 & .00 & .00 & .00 & .06 & .0004 \\
\hline & 89 & .0 & .0 & .0000 & .0000 & .00 & .00 & .00 & .0000 & .0003 \\
\hline & & ח & & 0000 & .0000 & .0000 & .0000 & .0000 & .0000 & .0003 \\
\hline & $9]$ & .0000 & .0 & .0000 & .0000 & .0000 & .0000 & .0000 & .0000 & .0003 \\
\hline & 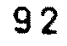 & & & 0 & .0000 & .00 & .00 & .00 & .0000 & .0002 \\
\hline & 93 & .0 & & .00 & .0000 & .0000 & .0000 & .00 & .0000 & .0002 \\
\hline & 94 & & & 0 & .0000 & .0000 & .0000 & .0000 & .0000 & .0002 \\
\hline & 95 & .0000 & .0000 & .0000 & .0000 & .0000 & .0000 & .0000 & .0000 & .0002 \\
\hline & 96 & .0000 & .0000 & .0000 & .0000 & .0000 & .0000 & .0000 & .0000 & .0002 \\
\hline & 97 & & & & 0 & 0 & & & 10 & \\
\hline
\end{tabular}


THETA $(\theta)$

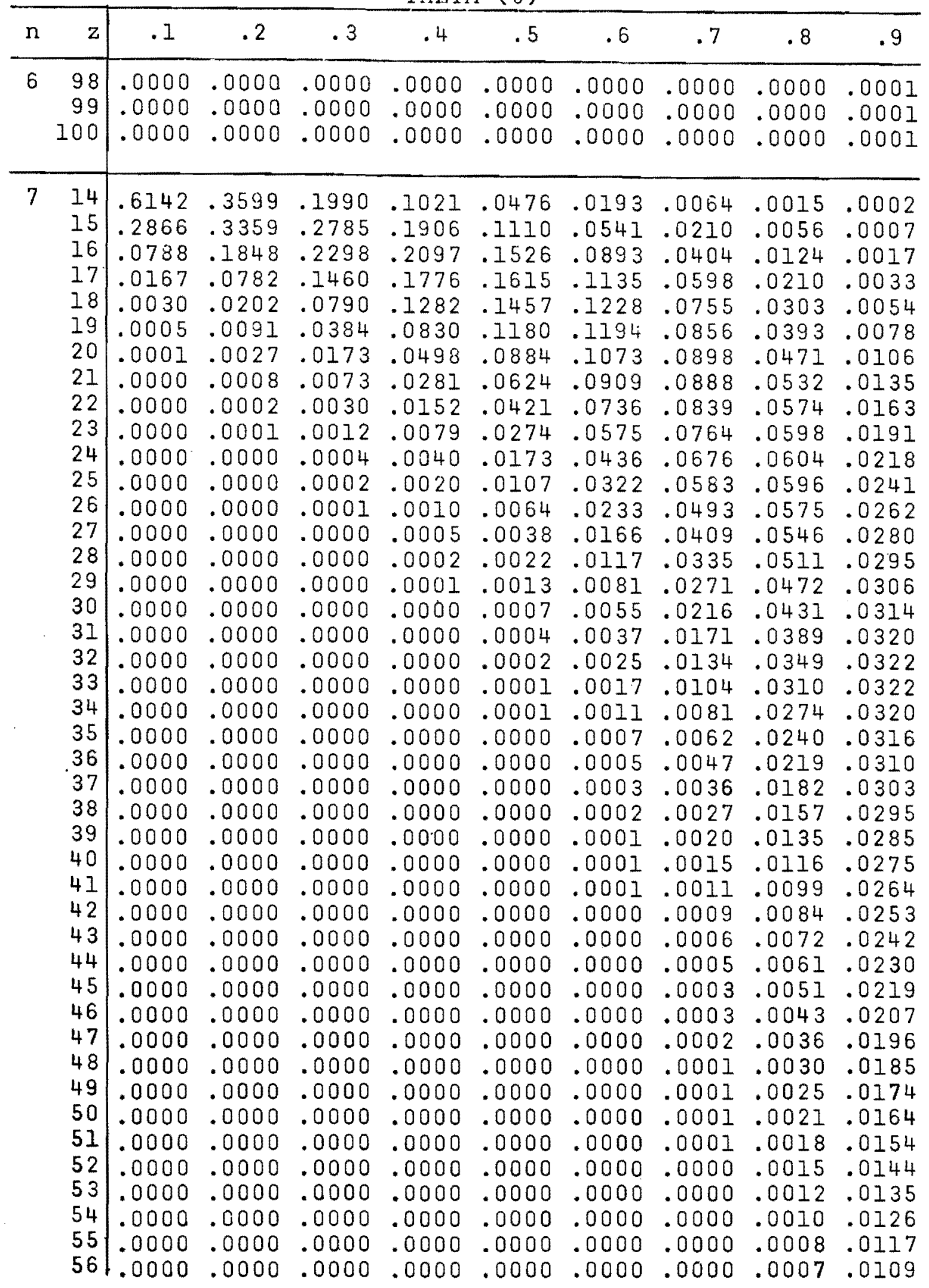


THETA $(\theta)$

\begin{tabular}{|c|c|c|c|c|c|c|c|c|c|c|}
\hline $\mathrm{n}$ & $\mathrm{z}$ & .1 & .2 & .3 & .4 & .5 & .6 & .7 & .8 & .9 \\
\hline 7 & 57 & 000 & & & & & & & .00 & .0102 \\
\hline & 58 & & & & & 0 & & 0 & .00 & . \\
\hline & 59 & 0000 & .0000 & .0000 & .0000 & .0000 & .0000 & .0000 & .0004 & .0088 \\
\hline & 60 & .0000 & .0000 & .0000 & .0000 & .0000 & & .0000 & .0003 & 081 \\
\hline & 61 & .0000 & .0000 & .0000 & .0000 & .0000 & & 0 & .0003 & \\
\hline & 62 & 0000 & .0000 & .0000 & .00 & .00 & 10 & .0000 & .0002 & .0070 \\
\hline & 63 & 0000 & .0000 & .0000 & .0000 & .00 & 0 & .00 & .0002 & .0065 \\
\hline & 64 & .0000 & .0000 & .0000 & .0000 & .0000 & .0000 & .0000 & .00 & .0060 \\
\hline & 65 & .0000 & .0000 & .0000 & .0000 & .0000 & .0000 & .0000 & .00 & .0055 \\
\hline & 66 & .0000 & .0000 & .0000 & .0000 & .0000 & .0 & .00 & .00 & .0051 \\
\hline & 67 & .0000 & .0000 & .0000 & .00 & .00 & & 0 & .0 & 0047 \\
\hline & 68 & .0000 & .0000 & .0000 & .00 & .00 & & 0 & .0 & .0043 \\
\hline & 69 & .0000 & .0000 & .00 & .0 & .00 & & & .0 & .0040 \\
\hline & 70 & .0000 & .0000 & .00 & .0 & .00 & & & .0 & .0037 \\
\hline & 71 & .0 & .0000 & .00 & .00 & .00 & & .00 & .00 & .0034 \\
\hline & 72 & .0000 & .0000 & .0000 & .00 & .0000 & .00 & .00 & .00 & .0031 \\
\hline & 73 & .0000 & .0000 & .0000 & .0000 & .0000 & .0 & .00 & .00 & .0029 \\
\hline & 74 & 0000 & .0000 & .0000 & .00 & .0000 & .0 & .0 & .0 & .0026 \\
\hline & 75 & 0 & .0000 & .00 & .00 & .00 & & .0 & .0 & .0024 \\
\hline & 76 & & .0000 & .0 & .0 & .00 & & & .00 & .0022 \\
\hline & 77 & 0 & .0 & .0 & .00 & .00 & & .00 & .00 & .0020 \\
\hline & 78 & .0000 & .0000 & .0000 & .0000 & .0000 & & .00 & .00 & .0019 \\
\hline & 79 & .0000 & .0000 & .0000 & .0000 & .0000 & & .0 & .00 & .0017 \\
\hline & 80 & 0000 & .0000 & .0000 & .0 & .00 & & & & .0016 \\
\hline & 81 & & .0000 & .0000 & & 0 & & & & .0014 \\
\hline & 82 & & .0000 & .0 & & .0 & & & & .0 \\
\hline & 83 & 0 & .0 & .0 & .0 & .00 & & & .00 & .0012 \\
\hline & 84 & 0 & .0000 & .0 & .0 & .00 & & & .0 & .0011 \\
\hline & 85 & .0000 & .0000 & .0000 & .0000 & .0000 & .0 & & .00 & .0010 \\
\hline & 86 & .0000 & .0000 & .0000 & .00 & .0000 & & & .0 & .0009 \\
\hline & 87 & 0 & .0000 & .00 & .0 & & & & & 08 \\
\hline & 88 & & .0 & & & & & & - & 0 \\
\hline & 89 & 0 & & .0 & & .0 & & & & .0007 \\
\hline & 90 & .0 & .0 & .0 & .0 & .00 & & & .0 & .0006 \\
\hline & 91 & .0 & .0000 & .0 & .0 & .00 & & & & .0006 \\
\hline & 92 & .0 & .0000 & & & .00 & & & & .0005 \\
\hline & 93 & .0000 & .0000 & .0 & .0 & .00 & & & .0 & .0005 \\
\hline & 94 & .0000 & .0000 & .0000 & .00 & .0000 & .0 & & .0 & .0004 \\
\hline & 95 & & .0000 & .0 & .0 & & & & .0 & .0004 \\
\hline & 96 & .00 & & .0 & & & & & .00 & .0004 \\
\hline & 97 & & & .0 & & & & & & .0003 \\
\hline & 98 & .00 & .0000 & .0000 & .0000 & .0000 & .0000 & & .00 & .0003 \\
\hline & 99 & .0000 & .0000 & .0000 & .0000 & .0000 & .0000 & .0 & .00 & .0003 \\
\hline & & .0000 & .0000 & .0000 & .0000 & .0000 & & 0 & .00 & .0002 \\
\hline & & .0000 & .0000 & .00 & .00 & 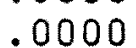 & .0 & & .00 & .0002 \\
\hline & & .0000 & .0000 & .0000 & & & & & .0 & .0002 \\
\hline & & .0000 & .0000 & .0000 & .0000 & .0000 & .0000 & .0000 & .0000 & .0002 \\
\hline
\end{tabular}


THETA $(\theta)$

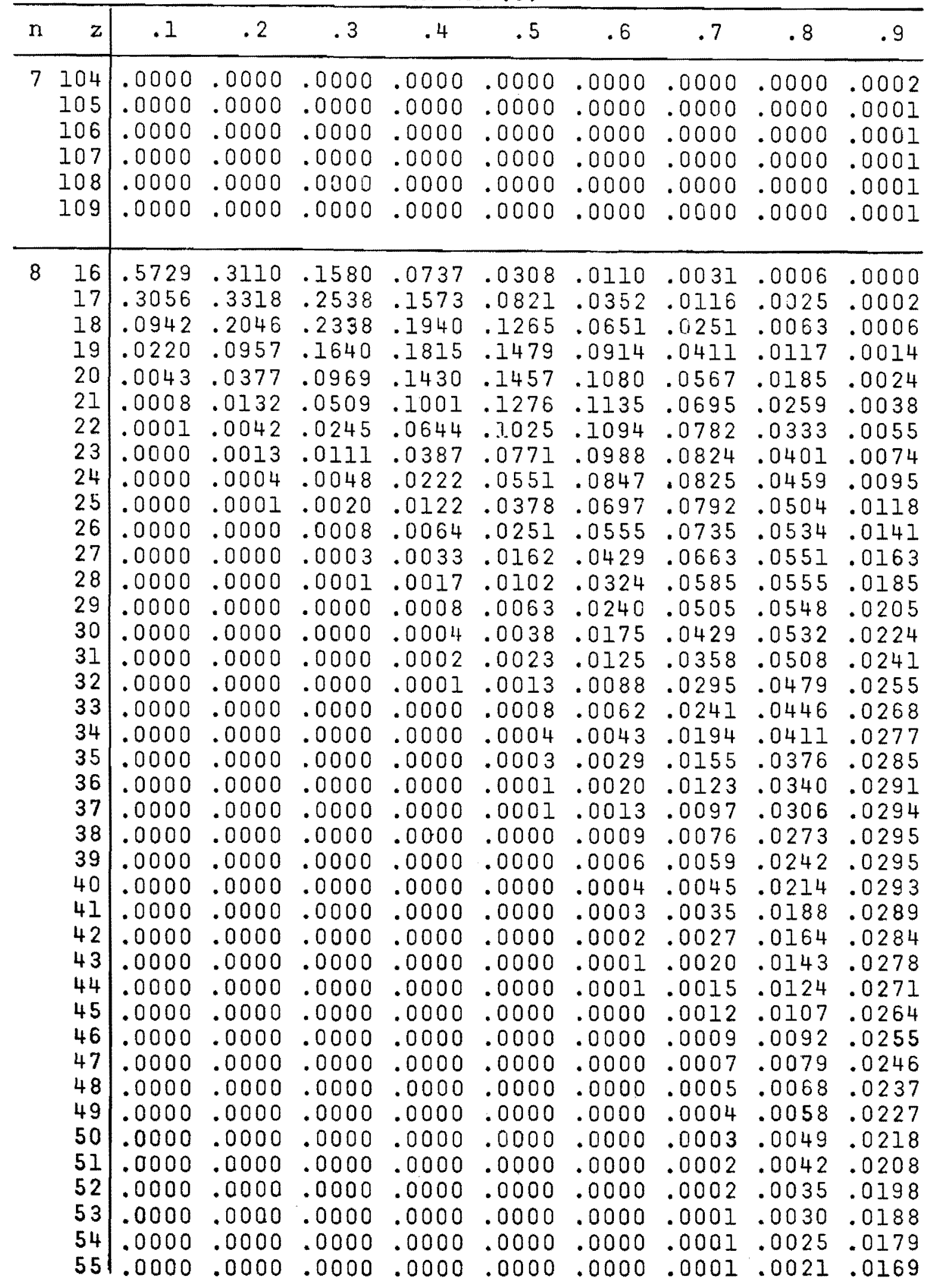


THETA $(\theta)$

\begin{tabular}{|c|c|c|c|c|c|c|c|c|c|c|}
\hline$n$ & $z$ & .1 & .2 & .3 & .4 & .5 & .6 & .7 & .8 & .9 \\
\hline 8 & 56 & .0000 & .0000 & 00 & .0000 & .0000 & .0000 & .0000 & .0018 & .0160 \\
\hline & 57 & .0000 & .0000 & .0000 & .0000 & .0000 & .0000 & .0000 & .0015 & .0151 \\
\hline & 58 & .0000 & .0000 & .0000 & .0000 & .0000 & .0000 & .0000 & .0012 & .0142 \\
\hline & 59 & .0000 & .0000 & .0000 & .0000 & .0000 & .0000 & .0000 & .0010 & .0134 \\
\hline & 60 & .0000 & .0000 & .0000 & .0000 & .0000 & .0000 & .0000 & .0009 & .0126 \\
\hline & 61 & .0000 & .0000 & .0000 & .0000 & .0000 & .0000 & .0000 & .0007 & .0118 \\
\hline & 62 & .0000 & .0000 & .0000 & .0000 & .0000 & .0000 & .0000 & .0006 & .0110 \\
\hline & 63 & .0000 & .0000 & .0000 & .0000 & .0000 & .0000 & .0000 & .0005 & .0103 \\
\hline & 64 & .0000 & .0000 & .0000 & .0000 & .0000 & .0000 & .0000 & .0004 & .0097 \\
\hline & 65 & .0000 & .0000 & .0000 & .0000 & .0000 & .0000 & .0000 & .0003 & .0090 \\
\hline & 66 & .0000 & .0000 & .0000 & .0000 & .0000 & .0000 & .0000 & .0003 & .0084 \\
\hline & 67 & .0000 & .0000 & .0000 & .0000 & .0000 & .0000 & .0000 & .0002 & .0078 \\
\hline & 68 & .0000 & .0000 & .0000 & .0000 & .0000 & .0000 & .0000 & .0002 & .0073 \\
\hline & 69 & .0000 & .0000 & .0000 & .0000 & .0000 & .0000 & .0000 & .0002 & .0068 \\
\hline & 70 & .0000 & .0000 & .0000 & .0000 & .0000 & .0000 & .0000 & .0001 & .0063 \\
\hline & 71 & .0000 & .0000 & .0000 & .0000 & .0000 & .0000 & .0000 & .0001 & .0059 \\
\hline & 72 & .0000 & .0000 & .0000 & .0000 & .0000 & .0000 & .0000 & .0000 & .0054 \\
\hline & 73 & .0000 & .0000 & .0000 & .0000 & .0000 & .0000 & .0000 & .0000 & .0050 \\
\hline & 74 & .0000 & .0000 & .0000 & .0000 & .0000 & .0000 & .0000 & .0000 & .0047 \\
\hline & 75 & .0000 & .0000 & .0000 & .0000 & .0000 & .0000 & .0000 & .0000 & .0043 \\
\hline & 76 & .0000 & .0000 & .0000 & .0000 & .0000 & .0000 & .0000 & .0000 & .0040 \\
\hline & 77 & .0000 & .0000 & .0000 & .0000 & .0000 & .0000 & .0000 & .0000 & .0037 \\
\hline & 78 & .0000 & .0000 & .0000 & .0000 & .0000 & .0000 & .0000 & .0000 & .0034 \\
\hline & 79 & .0000 & .0000 & .0 & .0000 & .0000 & .0000 & .0000 & .0000 & .0032 \\
\hline & 80 & .0000 & .0000 & .0000 & .0000 & .0000 & .0000 & .0000 & .0000 & .0029 \\
\hline & 81 & .0000 & .0000 & .0000 & .0000 & .0000 & .0000 & .0000 & .0000 & .0027 \\
\hline & 82 & .0000 & .0000 & .0000 & .0000 & .0000 & .0000 & .0000 & .0000 & .0025 \\
\hline & 83 & .0000 & .0000 & .0000 & .0000 & .0000 & .0000 & .0000 & .0000 & .0023 \\
\hline & 84 & .0000 & .0000 & .0000 & .0000 & .0000 & .0000 & .0000 & .0000 & .0021 \\
\hline & 85 & .0000 & .0000 & .0000 & .0000 & .0000 & .0000 & .0000 & .0000 & .0019 \\
\hline & 86 & .0000 & .0000 & .0000 & .0000 & .0000 & .0000 & .0000 & .0000 & .0018 \\
\hline & 87 & .0000 & .0000 & .0000 & .0000 & .0000 & .0000 & .0000 & .0000 & .0016 \\
\hline & 88 & .0000 & .0000 & .0000 & .0000 & .0000 & .0000 & .0000 & .0000 & .0015 \\
\hline & 89 & .0000 & .0000 & .0000 & .0000 & .0000 & .0000 & .0000 & .0000 & .0014 \\
\hline & 90 & .0000 & .0000 & .0000 & .0000 & .0000 & .0000 & .0000 & .0000 & .0013 \\
\hline & 91 & .0000 & .0000 & .0000 & .0000 & .0000 & .0000 & .0000 & .0000 & .0012 \\
\hline & 92 & .0000 & .0000 & .0000 & .0000 & .0000 & .0000 & .0000 & .0000 & .0011 \\
\hline & 93 & .0000 & .0000 & .0000 & .0000 & .0000 & .0000 & .0000 & .0000 & .0010 \\
\hline & 94 & .0000 & .0000 & .0000 & .0000 & .0000 & .0000 & .0000 & .0000 & .0009 \\
\hline & 95 & .0000 & .0000 & .0000 & .0000 & .0000 & .0000 & .0000 & .0000 & .0008 \\
\hline & 96 & .0000 & .0000 & .0000 & .0000 & .0000 & .0000 & .0000 & .0000 & .0008 \\
\hline & 97 & .0000 & .0000 & .0000 & .0000 & .0000 & .0000 & .0000 & .0000 & .0007 \\
\hline & 98 & .0000 & .0000 & .0000 & .0000 & .0000 & .0000 & .0000 & .0000 & .0006 \\
\hline & 99 & .0000 & .0000 & .0000 & .0000 & .0000 & .0000 & .0000 & .0000 & .0006 \\
\hline & & .0000 & .0000 & .0000 & .0000 & .0000 & .0000 & .0000 & .0000 & .0005 \\
\hline & 1 & .0000 & .0000 & .0000 & .0000 & .0000 & .0000 & .0000 & .0000 & .0005 \\
\hline & & 0000 & .0000 & .0000 & .0000 & .0000 & .0000 & .0000 & .0000 & .0004 \\
\hline
\end{tabular}


THETA $(\theta)$

\begin{tabular}{|c|c|c|c|c|c|c|c|c|c|c|}
\hline $\mathrm{n}$ & $\mathbf{z}$ & .1 & .2 & .3 & .4 & .5 & .6 & .7 & .8 & .9 \\
\hline 8 & $\begin{array}{l}103 \\
104 \\
105 \\
106 \\
107 \\
108 \\
109 \\
110 \\
111 \\
112 \\
113 \\
114 \\
115 \\
116 \\
117\end{array}$ & $\begin{array}{l}.0000 \\
.0000 \\
.0000 \\
.0000 \\
.0000 \\
.0000 \\
.0000 \\
.0000 \\
.0000 \\
.0000 \\
.0000 \\
.0000 \\
.0000\end{array}$ & $\begin{array}{l}.0000 \\
.0000 \\
.0000 \\
.0000 \\
.0000 \\
.0000 \\
.0000 \\
.0000 \\
.0000 \\
.0000 \\
.0000 \\
.0000 \\
.0000 \\
.0000 \\
.0000\end{array}$ & $\begin{array}{l}.0000 \\
.0000 \\
.0000 \\
.0000 \\
.0000 \\
.0000 \\
.0000 \\
.0000 \\
.0000 \\
.0000 \\
.0000 \\
.0000 \\
.0000 \\
.0000 \\
.0000\end{array}$ & $\begin{array}{l}.00 \\
.00 \\
.00 \\
.00 \\
.00 \\
.00 \\
.00 \\
.00 \\
.00 \\
.00 \\
.00 \\
.00 \\
.00\end{array}$ & $\begin{array}{l}.00 \\
.00 \\
.00 \\
.00 \\
.00 \\
.00 \\
.00 \\
.00 \\
.00 \\
.00 \\
.00 \\
.00 \\
.00\end{array}$ & $\begin{array}{l}.0000 \\
.0000 \\
.0000 \\
.0000 \\
.0000 \\
.0000 \\
.0000 \\
.0000 \\
.0000 \\
.0000 \\
.0000 \\
.0000 \\
.0000 \\
.0000 \\
.0000\end{array}$ & $\begin{array}{l}.00 \\
.00 \\
.00 \\
.00 \\
.00 \\
.00 \\
.00 \\
.00 \\
.00 \\
.00 \\
.00 \\
.00 \\
.00\end{array}$ & $\begin{array}{l}.00 \\
.00 \\
.00 \\
.00 \\
.00 \\
.00 \\
.00 \\
.00 \\
.00 \\
.00 \\
.00 \\
.00 \\
.00 \\
.00\end{array}$ & $\begin{array}{l}.0004 \\
.0004 \\
.0003 \\
.0003 \\
.0003 \\
.0003 \\
.0002 \\
.0002 \\
.0002 \\
.0002 \\
.0002 \\
.0001 \\
.0001 \\
.0001 \\
.0001\end{array}$ \\
\hline 9 & $\begin{array}{l}18 \\
19 \\
20 \\
21 \\
22 \\
23 \\
24 \\
25 \\
26 \\
27 \\
28 \\
29 \\
30 \\
31 \\
32 \\
33 \\
34 \\
35 \\
36 \\
37 \\
38 \\
39 \\
40 \\
41 \\
42 \\
43 \\
44 \\
45 \\
46 \\
47 \\
48\end{array}$ & $\begin{array}{l}.3206 \\
.1096 \\
.0281 \\
.0060 \\
.0011 \\
.0002 \\
.0000 \\
.0000 \\
.0000 \\
.0000 \\
.0000 \\
.0000 \\
.0000 \\
.0000 \\
.0000 \\
.0000 \\
.0000 \\
.0000 \\
.0000 \\
.0000 \\
.0000 \\
.0000 \\
.0000 \\
.0000 \\
.0000 \\
.0000 \\
.0000 \\
.0000 \\
.0000 \\
.0000\end{array}$ & $\begin{array}{l}.2688 \\
.3225 \\
.2204 \\
.1129 \\
.0482 \\
.0182 \\
.0062 \\
.0020 \\
.0006 \\
.0002 \\
.0000 \\
.0000 \\
.0000 \\
.0000 \\
.0000 \\
.0000 \\
.0000 \\
.0000 \\
.0000 \\
.0000 \\
.0000 \\
.0000 \\
.0000 \\
.0000 \\
.0000 \\
.0000 \\
.0000 \\
.0000 \\
.0000 \\
.0000 \\
.0000\end{array}$ & $\begin{array}{l}.2258 \\
.2314 \\
.1778 \\
.1139 \\
.0643 \\
.0331 \\
.0159 \\
.0072 \\
.0031 \\
.0013 \\
.0005 \\
.0002 \\
.0001 \\
.0000 \\
.0000 \\
.0000 \\
.0000 \\
.0000 \\
.0000 \\
.0000 \\
.0000 \\
.0000 \\
.0000 \\
.0000 \\
.0000 \\
.0000 \\
.0000 \\
.0000 \\
.0000 \\
.0000\end{array}$ & $\begin{array}{l}.1746 \\
.1778 \\
.1527 \\
.1150 \\
.0789 \\
.0504 \\
.0305 \\
.0176 \\
.0098 \\
.0053 \\
.0028 \\
.0014 \\
.0007 \\
.0004 \\
.0002 \\
.0001 \\
.0000 \\
.0000 \\
.0000 \\
.0000 \\
.0000 \\
.0000 \\
.0000 \\
.0000 \\
.0000 \\
.0000 \\
.0000 \\
.0000 \\
.0000\end{array}$ & $\begin{array}{l}.1127 \\
.0900 \\
.0680 \\
.0490 \\
.0341 \\
.0229 \\
.0150 \\
.0096 \\
.0061 \\
.0037 \\
.0023 \\
.0014 \\
.0008 \\
.0005 \\
.0003 \\
.0002 \\
.0001 \\
.0001 \\
.0000 \\
.0000 \\
.0000 \\
.0000 \\
.0000 \\
.0000 \\
.0000\end{array}$ & $\begin{array}{l}.0063 \\
.0225 \\
.0462 \\
.0710 \\
.0910 \\
.1027 \\
.1058 \\
.0014 \\
.0919 \\
.0796 \\
.0663 \\
.0536 \\
.0422 \\
.0324 \\
.0244 \\
.0181 \\
.0132 \\
.0095 \\
.0068 \\
.0048 \\
.0033 \\
.0023 \\
.0016 \\
.0011 \\
.0007 \\
.0005 \\
.0003 \\
.0002 \\
.0001 \\
.0001 \\
.0001\end{array}$ & $\begin{array}{l}.0015 \\
.0064 \\
.0152 \\
.0273 \\
.0408 \\
.0538 \\
.0646 \\
.0723 \\
.0764 \\
.0772 \\
.0750 \\
.0707 \\
.0649 \\
.0582 \\
.0512 \\
.0443 \\
.0377 \\
.0316 \\
.0263 \\
.0216 \\
.0175 \\
.0141 \\
.0113 \\
.0090 \\
.0071 \\
.0055 \\
.0043 \\
.0034 \\
.0026 \\
.0020 \\
.0015\end{array}$ & $\begin{array}{l}.0002 \\
.0011 \\
.0031 \\
.0063 \\
.0108 \\
.0163 \\
.0224 \\
.0286 \\
.0346 \\
.0399 \\
.0444 \\
.0478 \\
.0501 \\
.0514 \\
.0517 \\
.0510 \\
.0496 \\
.0476 \\
.0452 \\
.0424 \\
.0394 \\
.0363 \\
.0332 \\
.0301 \\
.0271 \\
.0243 \\
.0217 \\
.0192 \\
.0169 \\
.0149 \\
.0130\end{array}$ & $\begin{array}{l}.0000 \\
.0001 \\
.0002 \\
.0005 \\
.0010 \\
.0017 \\
.0027 \\
.0039 \\
.0052 \\
.0068 \\
.0085 \\
.0103 \\
.0122 \\
.0141 \\
.0159 \\
.0177 \\
.0193 \\
.0209 \\
.0223 \\
.0235 \\
.0246 \\
.0255 \\
.0262 \\
.0267 \\
.0271 \\
.0273 \\
.0274 \\
.0273 \\
.0271 \\
.0268 \\
.0264\end{array}$ \\
\hline
\end{tabular}


THETA $(\theta)$

\begin{tabular}{|c|c|c|c|c|c|c|c|c|c|c|}
\hline$n$ & z) & .1 & .2 & .3 & .4 & .5 & .6 & .7 & .8 & .9 \\
\hline \multirow[t]{47}{*}{9} & 49 & .0000 & 0000 & 0000 & 0000 & .0000 & .0000 & .0012 & .0114 & .025 \\
\hline & $\begin{array}{l}50 \\
57\end{array}$ & - & .00 & . 0 & .0000 & .0000 & .000 & & & \\
\hline & 51 & .0000 & - & .0000 & .0000 & & & & & \\
\hline & 52 & .0000 & .0000 & .0000 & .0000 & .0000 & .0000 & .0005 & .0074 & .0239 \\
\hline & 53 & .0000 & .0000 & .0000 & .0000 & .0000 & .0000 & .0004 & .0063 & .0232 \\
\hline & 54 & .0000 & .0000 & .0000 & .0000 & .0000 & .0000 & .0003 & .0054 & .0224 \\
\hline & 55 & .0000 & .0000 & .0000 & .0000 & .0000 & .0000 & .0002 & .0047 & .0215 \\
\hline & 56 & .0000 & .0000 & .0000 & .0000 & .0000 & .0000 & .0002 & .0040 & .0207 \\
\hline & 57 & .0000 & .0000 & .0000 & .0000 & .0000 & .0000 & .0001 & .0034 & .0198 \\
\hline & 58 & 100 & .0000 & .0000 & .0000 & .0000 & .0000 & .0001 & .0029 & .0190 \\
\hline & 59 & .0000 & .0000 & .0000 & .0000 & .0000 & .0000 & .0001 & .0024 & .0181 \\
\hline & 60 & .0000 & .0000 & .0000 & .0000 & .0000 & .0000 & .0000 & .0021 & .017 \\
\hline & 61 & .0000 & .0000 & .0000 & .0000 & .0000 & .0000 & .0000 & .0018 & .0164 \\
\hline & 62 & .0000 & .0000 & .0000 & .0000 & .0000 & .0000 & .0000 & .0015 & .015 \\
\hline & 63 & .0000 & .0000 & .0000 & .0000 & .0000 & .0000 & .0000 & .0012 & .0148 \\
\hline & 64 & .0000 & .0000 & .0000 & .0000 & .0000 & .0000 & .0000 & .0010 & .0140 \\
\hline & 65 & .0000 & .0000 & .0000 & .0000 & .0000 & .0000 & .0000 & .0009 & .0132 \\
\hline & 66 & .0000 & .0000 & 0000 & .0000 & .0000 & .0000 & .0000 & .0007 & 0725 \\
\hline & 67 & .0000 & .0000 & .0000 & .0000 & .0000 & .0000 & .0000 & .0006 & .0118 \\
\hline & 68 & .0000 & .0000 & .0000 & .0000 & .0000 & .0000 & .0000 & .0005 & .0111 \\
\hline & 69 & .0000 & .0000 & .0000 & .0000 & .0000 & .0000 & .0000 & .0004 & .01 \\
\hline & 70 & .0000 & .0000 & .0000 & .0000 & .0000 & .0000 & .0000 & .0004 & .0098 \\
\hline & 71 & .0000 & .0000 & 0000 & .0000 & .0000 & .00 & .0000 & .0003 & .00 \\
\hline & 72 & .0000 & .0000 & .0000 & .0000 & .0000 & .0000 & .0000 & .0002 & .0086 \\
\hline & 73 & .0000 & .0000 & 0000 & .0000 & .00 & .00 & & .0002 & \\
\hline & 74 & .0000 & .0000 & .0000 & .0000 & .0000 & .0000 & .0000 & .0002 & .0075 \\
\hline & 75 & .0000 & .00 & 00 & .00 & .00 & .06 & & & \\
\hline & 76 & .0000 & .0000 & .0000 & .0000 & .0000 & .0000 & .0000 & .0001 & .0066 \\
\hline & 77 & .0000 & .0000 & .00 & & .0 & .0000 & .0 & .0001 & .0061 \\
\hline & 78 & .0000 & .0000 & .0000 & .0000 & .0000 & .0000 & .0000 & .0000 & 57 \\
\hline & 79 & .0000 & .0000 & .0000 & .0000 & .0000 & .0000 & .0000 & .0000 & .0053 \\
\hline & 8 & & 0000 & 0000 & & & & & & \\
\hline & 81 & .0000 & .0000 & .0000 & .0000 & .0000 & .0000 & .0000 & .0000 & .0046 \\
\hline & 8 & & & & & & & & & \\
\hline & 83 & .0000 & .0000 & .0000 & .0000 & .0000 & .0000 & .0000 & .0000 & .0040 \\
\hline & 84 & .00 & .00 & & & & .0 & & & \\
\hline & 85 & .0000 & .0000 & .0000 & .0000 & .00 & .0000 & .06 & .0000 & .0034 \\
\hline & 8 & .00 & 00 & 00 & .00 & .00 & .00 & & .0 & .0032 \\
\hline & 87 & .0 & .0000 & .0000 & .0000 & .0000 & .0000 & .00 & .0000 & .0029 \\
\hline & 8 & & & & & & .00 & & .00 & .00 \\
\hline & 89 & .00 & .0000 & .0000 & .0000 & .00 & .0000 & .00 & .0000 & .0025 \\
\hline & 90 & .0000 & .0000 & .0000 & .0000 & .0000 & .0000 & .0000 & .0000 & .0023 \\
\hline & 91 & .0000 & .0000 & .0000 & .0000 & .0000 & .0000 & & .0000 & .002 \\
\hline & 92 & & 0000 & 0000 & .0000 & .0000 & .0000 & .0 & .00 & .002 \\
\hline & 93 & .0000 & 0000 & .0000 & .0000 & .0000 & .0000 & .0000 & .0000 & .001 \\
\hline & 94 & .0000 & .0000 & .0000 & .0000 & .0000 & .0000 & .0000 & .0000 & .0017 \\
\hline & & & & & & & & & & \\
\hline
\end{tabular}


THETA $(\theta)$

\begin{tabular}{|c|c|c|c|c|c|c|c|c|c|c|}
\hline $\mathrm{n}$ & $\mathbf{z}$ & .1 & .2 & .3 & .4 & .5 & 6 & .7 & .8 & .9 \\
\hline 9 & $\begin{array}{r}96 \\
97 \\
98 \\
99 \\
100 \\
101 \\
102 \\
103 \\
104 \\
105 \\
106 \\
107 \\
108 \\
109 \\
110 \\
111 \\
112 \\
113 \\
114 \\
115 \\
116 \\
117 \\
118 \\
119 \\
120 \\
121 \\
122 \\
123 \\
124 \\
125\end{array}$ & $\begin{array}{l}.0000 \\
.0000 \\
.0000 \\
.0000 \\
.0000 \\
.0000 \\
.0000 \\
.0000 \\
.0000 \\
.0000 \\
.0000 \\
.0000 \\
.0000 \\
.0000 \\
.0000 \\
.0000 \\
.0000 \\
.0000 \\
.0000 \\
.0000 \\
.0000 \\
.0000 \\
.0000 \\
.0000 \\
.0000 \\
.0000 \\
.0000 \\
.0000 \\
.0000 \\
.0000\end{array}$ & $\begin{array}{l}.0000 \\
.0000 \\
.0000 \\
.0000 \\
.0000 \\
.0000 \\
.0000 \\
.0000 \\
.0000 \\
.0000 \\
.0000 \\
.0000 \\
.0000 \\
.0000 \\
.0000 \\
.0000 \\
.0000 \\
.0000 \\
.0000 \\
.0000 \\
.0000 \\
.0000 \\
.0000 \\
.0000 \\
.0000 \\
.0000 \\
.0000 \\
.0000 \\
.0000\end{array}$ & $\begin{array}{l}.00 \\
.00 \\
.00 \\
.00 \\
.00 \\
.00 \\
.00 \\
.00 \\
.00 \\
.00 \\
.00 \\
.00 \\
.00 \\
.00 \\
.00 \\
.00 \\
.00 \\
.00 \\
.00 \\
.00\end{array}$ & $\begin{array}{l}.00 \\
.00 \\
.00 \\
.00 \\
.00 \\
.00 \\
.00 \\
.00 \\
.00 \\
.00 \\
.00 \\
.00 \\
.00 \\
.00 \\
.00 \\
.00 \\
.00 \\
.00 \\
.00 \\
.00\end{array}$ & $\begin{array}{l}.00 \\
.00 \\
.00 \\
.00 \\
.00 \\
.00 \\
.00 \\
.00 \\
.00 \\
.00 \\
.00 \\
.00 \\
.00 \\
.00 \\
.00 \\
.00 \\
.00 \\
.00 \\
.00 \\
.00 \\
.00 \\
.00 \\
.00 \\
.00 \\
.00 \\
.00 \\
.00 \\
.00\end{array}$ & $\begin{array}{l}0 \\
0 \\
0 \\
0 \\
0 \\
0 \\
0 \\
0 \\
0 \\
0 \\
0 \\
0 \\
0 \\
0 \\
0 \\
0 \\
0 \\
0 \\
0 \\
0 \\
0 \\
0 \\
0 \\
0 \\
0 \\
0 \\
0 \\
0\end{array}$ & $\begin{array}{l}0 \\
0 \\
0 \\
0 \\
0 \\
0 \\
0 \\
0 \\
0 \\
0 \\
0 \\
0 \\
0 \\
0 \\
0 \\
0 \\
0 \\
0 \\
0 \\
0 \\
0 \\
0 \\
0\end{array}$ & $\begin{array}{l}000 \\
000 \\
000 \\
000 \\
000 \\
000 \\
000 \\
000 \\
000 \\
000 \\
000 \\
000 \\
000 \\
0000 \\
000 \\
0000 \\
1000 \\
1000 \\
0000 \\
1000 \\
1000 \\
1000 \\
1000 \\
1000 \\
0000 \\
1000 \\
0000 \\
1000 \\
1000 \\
1000\end{array}$ & $\begin{array}{l}.0 \\
.0 \\
.0 \\
.0 \\
.0 \\
.0 \\
.0 \\
.0 \\
.0\end{array}$ \\
\hline & $\begin{array}{l}20 \\
21 \\
22 \\
23 \\
24 \\
25 \\
26 \\
27 \\
28 \\
29 \\
30 \\
31 \\
32 \\
33 \\
34 \\
35\end{array}$ & $\begin{array}{l}.4985 \\
.3323 \\
.1246 \\
.0347 \\
.0080 \\
.0016 \\
.0003 \\
.0000 \\
.0000 \\
.0000 \\
.0000 \\
.0000 \\
.0000 \\
.0000 \\
.0000 \\
.0000\end{array}$ & $\begin{array}{l}.2323 \\
.1292 \\
.0595 \\
.0240 \\
.0088 \\
.0030 \\
.0009 \\
.0003 \\
.0001 \\
.0000 \\
.0000 \\
.0000 \\
.0000 \\
.0000\end{array}$ & $\begin{array}{l}.0996 \\
.1992 \\
.2241 \\
.1870 \\
.1291 \\
.0780 \\
.0427 \\
.0217 \\
.0103 \\
.0047 \\
.0021 \\
.0009 \\
.0004 \\
.0001 \\
.0001 \\
.0000\end{array}$ & $\begin{array}{r}.1710 \\
.1574 \\
.1269 \\
.0926 \\
.0626 \\
.0399 \\
.0242 \\
.0140 \\
.0079 \\
.0043 \\
.0023 \\
.0012 \\
.0006\end{array}$ & $\begin{array}{l}.0129 \\
.0430 \\
.0806 \\
.1121 \\
.1289 \\
.1299 \\
.1185 \\
.1002 \\
.0797 \\
.0604 \\
.0439 \\
.0308 \\
.0210 \\
.0140 \\
.0091 \\
.0058\end{array}$ & $\begin{array}{l}.0036 \\
.0143 \\
.0321 \\
.0535 \\
.0739 \\
.0894 \\
.0979 \\
.0993 \\
.0948 \\
.0861 \\
.0751 \\
.0633 \\
.0518 \\
.0413 \\
.0322 \\
.0247\end{array}$ & $\begin{array}{l}.0176 \\
.0283 \\
.0406 \\
.0511 \\
.0604 \\
.0673 \\
.0714 \\
.0726 \\
.0714 \\
.0818 \\
.0634 \\
.0577 \\
.0515\end{array}$ & $\begin{array}{l}.0001 \\
.0005 \\
.0015 \\
.0033 \\
.0061 \\
.0099 \\
.0144 \\
.0195 \\
.0248 \\
.0300 \\
.0349 \\
.0392 \\
.0428 \\
.0455 \\
.0473 \\
.0483\end{array}$ & $\begin{array}{r}.0000 \\
.0000 \\
.0001 \\
.0002 \\
.0004 \\
.0008 \\
.0013 \\
.0019 \\
.0027 \\
.0037 \\
.0049 \\
.0062 \\
.0076 \\
.0091 \\
.0106 \\
.0122\end{array}$ \\
\hline
\end{tabular}


THETA ( $\theta)$

\begin{tabular}{|c|c|c|c|c|c|c|c|c|c|c|}
\hline $\mathrm{n}$ & $z$ & .1 & .2 & .3 & .4 & .5 & .6 & .7 & .8 & .9 \\
\hline \multirow[t]{48}{*}{10} & 36 & .0000 & .0000 & .0000 & .0006 & .0036 & .0186 & .0453 & .0485 & .0138 \\
\hline & 37 & .0 & & .0000 & .0002 & .0022 & .0138 & .0391 & .0479 & .0153 \\
\hline & 38 & .0000 & .0000 & .0000 & .0001 & .0014 & .0101 & .0334 & .0467 & .0168 \\
\hline & 39 & .0000 & .0000 & .0000 & .0000 & .0008 & .0073 & .0281 & .0450 & .0182 \\
\hline & 40 & .0000 & .0000 & .0000 & .0000 & .0005 & .0052 & .0235 & .0429 & .0195 \\
\hline & 41 & .0000 & .0000 & .0000 & .0000 & .0003 & .0037 & .0194 & .0405 & .0208 \\
\hline & 42 & .0000 & .0000 & .0000 & .0000 & .0002 & .0026 & .0159 & .0379 & .0218 \\
\hline & 43 & .0000 & .0000 & .0000 & .0000 & .0001 & .0018 & .0129 & .0351 & .0228 \\
\hline & 44 & .0000 & .0000 & .0000 & .0000 & .0001 & .0012 & .0104 & .0323 & .0236 \\
\hline & 45 & .0000 & .0000 & .0000 & .0000 & .0000 & .0008 & .0083 & .0296 & .0243 \\
\hline & 46 & .0000 & .0000 & .0000 & .0000 & .0000 & .0006 & .0066 & .0269 & .0248 \\
\hline & 47 & .0000 & .0000 & .0000 & .0000 & .0000 & .0004 & .0052 & .0243 & .0252 \\
\hline & 48 & .0000 & .0000 & .0000 & .0000 & .0000 & .0003 & .0041 & .0218 & .0255 \\
\hline & 49 & .0000 & .0000 & .0000 & .0000 & .0000 & .0002 & .0032 & .0195 & .0256 \\
\hline & 50 & .0000 & .0000 & .0000 & .0000 & .0000 & .0001 & .0025 & .0173 & .0256 \\
\hline & 51 & .0000 & .0000 & .0000 & .0000 & .0000 & .0001 & .0019 & .01 & .0256 \\
\hline & 52 & .0000 & .0000 & .0000 & .0000 & .0000 & .0001 & .0015 & .0135 & .0254 \\
\hline & 53 & .0000 & .0000 & .0000 & .0000 & .0000 & .0000 & .0011 & .0119 & .0251 \\
\hline & 54 & .0000 & .0000 & .0000 & .0000 & .0000 & .0000 & .0009 & .0104 & .0247 \\
\hline & 55 & .0000 & .0000 & .0000 & .0000 & .0000 & .0000 & .0007 & .0091 & .0243 \\
\hline & 56 & .0000 & .0000 & .0000 & .0000 & .0000 & .0000 & .0005 & .0079 & .0238 \\
\hline & 57 & .0000 & .0000 & .0000 & .0000 & .0000 & .0000 & .0004 & .0069 & .0232 \\
\hline & 58 & .0000 & .0000 & .0000 & .0000 & .0000 & .0000 & .0003 & .0059 & .0226 \\
\hline & 59 & .0000 & .0000 & .0000 & .0000 & .0000 & .0000 & .0002 & .0051 & .0219 \\
\hline & 60 & .0000 & .0000 & .0000 & .0000 & .0000 & .0000 & .0001 & .0044 & .0212 \\
\hline & 61 & .0000 & .0000 & .0000 & .0000 & .0000 & .0000 & .0001 & .0038 & .0205 \\
\hline & 62 & .0000 & .0000 & .0000 & .0000 & .0000 & .0000 & .0001 & .0033 & .0198 \\
\hline & 63 & .0000 & .0000 & .0000 & .0000 & .0000 & .0000 & .0000 & .0028 & .0190 \\
\hline & 64 & .0000 & .0000 & .0000 & .0000 & .0000 & .0000 & .0000 & $: 0024$ & .0183 \\
\hline & 65 & .0000 & .0000 & .0000 & .0000 & .0000 & .0000 & .0000 & .0020 & .0175 \\
\hline & 66 & .0000 & .0000 & .0000 & .0000 & .0000 & .0000 & .0000 & .0017 & .0167 \\
\hline & 67 & .0000 & .0000 & .0000 & .0000 & .0000 & .0000 & .0000 & .0015 & .0160 \\
\hline & 68 & .0000 & .0000 & .0000 & .0000 & .0000 & .0000 & .0000 & .0012 & .0152 \\
\hline & 69 & .0000 & .0000 & .0000 & .0000 & .0000 & .0000 & .0000 & .0010 & .0145 \\
\hline & 70 & .0000 & .0000 & .0000 & .0000 & .0000 & .0000 & .0000 & .0009 & .0138 \\
\hline & 71 & .0000 & .0000 & .0000 & .0000 & .0000 & .0000 & .0000 & .0007 & .0131 \\
\hline & 72 & .0000 & .0000 & .0000 & .0000 & .0000 & .0000 & .0000 & .0006 & .0124 \\
\hline & 73 & .0000 & .0000 & .0000 & .0000 & .0000 & .0000 & .0000 & .0005 & .0117 \\
\hline & 74 & .0000 & .0000 & .0000 & .0000 & .0000 & .0000 & .0000 & .0004 & .0111 \\
\hline & 75 & .0000 & .0000 & .0000 & .0000 & .0000 & .0000 & .0000 & .0004 & .0105 \\
\hline & 76 & .0000 & .0000 & .0000 & .0000 & .0000 & .0000 & .0000 & .0003 & .0099 \\
\hline & 77 & .0000 & .0000 & .0000 & .0000 & .0000 & .0000 & .0000 & .0003 & .0093 \\
\hline & 78 & .0000 & .0000 & .0000 & .0000 & .0000 & .0000 & .0000 & .0002 & .0087 \\
\hline & 79 & .0000 & .0000 & .0000 & .0000 & .0000 & .0000 & .0000 & .0002 & .0082 \\
\hline & 80 & .0000 & .0000 & .0000 & .0000 & .0000 & .0000 & .0000 & .0001 & .0077 \\
\hline & 81 & .0000 & .0000 & .0000 & .0000 & .0000 & .0000 & .0000 & .0001 & .0072 \\
\hline & 82 & .0000 & .0000 & .0000 & .0000 & .0000 & .0000 & .0000 & .0001 & .0068 \\
\hline & 83 & .0000 & .0000 & .0000 & .0000 & .0000 & .0000 & .0000 & .0000 & .0063 \\
\hline
\end{tabular}


THETA (S)

\begin{tabular}{|c|c|c|c|c|c|c|c|c|c|c|}
\hline $\mathrm{n}$ & $z$ & .1 & .2 & .3 & .4 & .5 & .6 & .7 & .8 & .9 \\
\hline 1 & $\begin{array}{l}84 \\
85 \\
86 \\
87 \\
88 \\
89 \\
90 \\
91 \\
92 \\
93 \\
94 \\
95 \\
96 \\
97 \\
98 \\
99 \\
100 \\
101 \\
102 \\
103 \\
104 \\
105 \\
106 \\
107 \\
108 \\
109 \\
110 \\
111 \\
112 \\
113 \\
114 \\
115 \\
116 \\
117 \\
118 \\
119 \\
120 \\
21 \\
122 \\
123 \\
24 \\
25 \\
26 \\
27 \\
28 \\
29 \\
30\end{array}$ & $\begin{array}{l}.0000 \\
.000 .0 \\
.0000 \\
.0000 \\
.00 .00 \\
.0000 \\
.0000 \\
.0000 \\
.0000 \\
.0000 \\
.0000 \\
.0000 \\
.0000 \\
.0000 \\
.0000 \\
.0000 \\
.0000 \\
.0000 \\
.0000 \\
.0000 \\
.0000 \\
.0000 \\
.0000 \\
.0000 \\
.0000 \\
.0000 \\
.0000 \\
.0000 \\
.0000 \\
.0000 \\
.0000 \\
.0000 \\
.0000 \\
.0000 \\
.0000 \\
.0000 \\
.0000 \\
.0000 \\
.0000 \\
.0000 \\
.0000 \\
.0000 \\
.0000 \\
.0000 \\
.0000 \\
.0000 \\
.0000\end{array}$ & $\begin{array}{l}.0000 \\
.0000 \\
.000 .0 \\
.0000 \\
.0000 \\
.0000 \\
.0000 \\
.0000 \\
.0000 \\
.0000 \\
.0000 \\
.0000 \\
.0000 \\
.0000 \\
.0000 \\
.0000 \\
.0000 \\
.0000 \\
.0000 \\
.0000 \\
.0000 \\
.0000 \\
.0000 \\
.0000 \\
.0000 \\
.0000 \\
.0000 \\
.0000 \\
.0000 \\
.0000 \\
.0000 \\
.0000 \\
.0000 \\
.0000 \\
.0000 \\
.0000 \\
.0000 \\
.0000 \\
.0000 \\
.0000 \\
.0000 \\
.0000 \\
.0000 \\
.0000 \\
.0000 \\
.0000 \\
.0000\end{array}$ & $\begin{array}{l}.0000 \\
.0000 \\
.0000 \\
.0000 \\
.0000 \\
.0000 \\
.0000 \\
.0000 \\
.0000 \\
.0000 \\
.0000 \\
.0000 \\
.0000 \\
.0000 \\
.0000 \\
.0000 \\
.0000 \\
.0000 \\
.0000 \\
.0000 \\
.0000 \\
.0000 \\
.0000 \\
.0000 \\
.0000 \\
.0000 \\
.0000 \\
.0000 \\
.0000 \\
.0000 \\
.0000 \\
.0000 \\
.0000 \\
.0000 \\
.0000 \\
.0000 \\
.0000 \\
.0000 \\
.0000 \\
.0000 \\
.0000 \\
.0000 \\
.0000 \\
.0000 \\
.0000 \\
.0000 \\
.0000\end{array}$ & $\begin{array}{l}.0000 \\
.0000 \\
.0000 \\
.0000 \\
.0000 \\
.0000 \\
.0000 \\
.0000 \\
.0000 \\
.0000 \\
.0000 \\
.0000 \\
.0000 \\
.0000 \\
.0000 \\
.0000 \\
.0000 \\
.0000 \\
.0000 \\
.0000 \\
.0000 \\
.0000 \\
.0000 \\
.0000 \\
.0000 \\
.0000 \\
.0000 \\
.0000 \\
.0000 \\
.0000 \\
.0000 \\
.0000 \\
.0000 \\
.0000 \\
.0000 \\
.0000 \\
.0000 \\
.0000 \\
.0000 \\
.0000 \\
.0000 \\
.0000 \\
.0000 \\
.0000 \\
.0000 \\
.0000 \\
.0000\end{array}$ & $\begin{array}{l}.0000 \\
.0000 \\
.0000 \\
.0000 \\
.0000 \\
.0000 \\
.0000 \\
.0000 \\
.0000 \\
.0000 \\
.0000 \\
.0000 \\
.0000 \\
.0000 \\
.0000 \\
.0000 \\
.0000 \\
.0000 \\
.0000 \\
.0000 \\
.0000 \\
.0000 \\
.0000 \\
.0000 \\
.0000 \\
.0000 \\
.0000 \\
.0000 \\
.0000 \\
.0000 \\
.0000 \\
.0000 \\
.0000 \\
.0000 \\
.0000 \\
.0000 \\
.0000 \\
.0000 \\
.0000 \\
.0000 \\
.0000 \\
.0000 \\
.0000 \\
.0000 \\
.0000 \\
.0000 \\
.0000\end{array}$ & $\begin{array}{l}.0000 \\
.0000 \\
.0000 \\
.0000 \\
.0000 \\
.0000 \\
.0000 \\
.0000 \\
.0000 \\
.0000 \\
.0000 \\
.0000 \\
.0000 \\
.0000 \\
.0000 \\
.0000 \\
.0000 \\
.0000 \\
.0000 \\
.0000 \\
.0000 \\
.0000 \\
.0000 \\
.0000 \\
.0000 \\
.0000 \\
.0000 \\
.0000 \\
.0000 \\
.0000 \\
.0000 \\
.0000 \\
.0000 \\
.0000 \\
.0000 \\
.0000 \\
.0000 \\
.0000 \\
.0000 \\
.0000 \\
.0000 \\
.0000 \\
.0000 \\
.0000 \\
.0000 \\
.0000 \\
.0000\end{array}$ & $\begin{array}{l}.0000 \\
.0000 \\
.0000 \\
.0000 \\
.0000 \\
.0000 \\
.0000 \\
.0000 \\
.0000 \\
.0000 \\
.0000 \\
.0000 \\
.0000 \\
.0000 \\
.0000 \\
.0000 \\
.0000 \\
.0000 \\
.0000 \\
.0000 \\
.0000 \\
.0000 \\
.0000 \\
.0000 \\
.0000 \\
.0000 \\
.0000 \\
.0000 \\
.0000 \\
.0000 \\
.0000 \\
.0000 \\
.0000 \\
.0000 \\
.0000 \\
.0000 \\
.0000 \\
.0000 \\
.0000 \\
.0000 \\
.0000 \\
.0000 \\
.0000 \\
.0000 \\
.0000 \\
.0000 \\
.0000\end{array}$ & $\begin{array}{l}.0000 \\
.0000 \\
.0000 \\
.0000 \\
.0000 \\
.0000 \\
.0000 \\
.0000 \\
.0000 \\
.0000 \\
.0000 \\
.0000 \\
.0000 \\
.0000 \\
.0000 \\
.0000 \\
.0000 \\
.0000 \\
.0000 \\
.0000 \\
.0000 \\
.0000 \\
.0000 \\
.0000 \\
.0000 \\
.0000 \\
.0000 \\
.0000 \\
.0000 \\
.0000 \\
.0000 \\
.0000 \\
.0000 \\
.0000 \\
.0000 \\
.0000 \\
.0000 \\
.0000 \\
.0000 \\
.0000 \\
.0000 \\
.0000 \\
.0000 \\
.0000 \\
.0000 \\
.0000 \\
.0000\end{array}$ & $\begin{array}{l}.0059 \\
.0055 \\
.0052 \\
.0048 \\
.0045 \\
.0042 \\
.0039 \\
.0035 \\
.0034 \\
.0032 \\
.0029 \\
.0027 \\
.0025 \\
.0023 \\
.0022 \\
.0020 \\
.0019 \\
.0017 \\
.0016 \\
.0015 \\
.0014 \\
.0013 \\
.0012 \\
.0011 \\
.0010 \\
.0009 \\
.0008 \\
.0008 \\
.0007 \\
.0007 \\
.0006 \\
.0006 \\
.0005 \\
.0005 \\
.0004 \\
.0004 \\
.0004 \\
.0003 \\
.0003 \\
.0003 \\
.0003 \\
.0002 \\
.0002 \\
.0002 \\
.0002 \\
.0002 \\
.0001\end{array}$ \\
\hline
\end{tabular}


THE'TA $(\theta)$

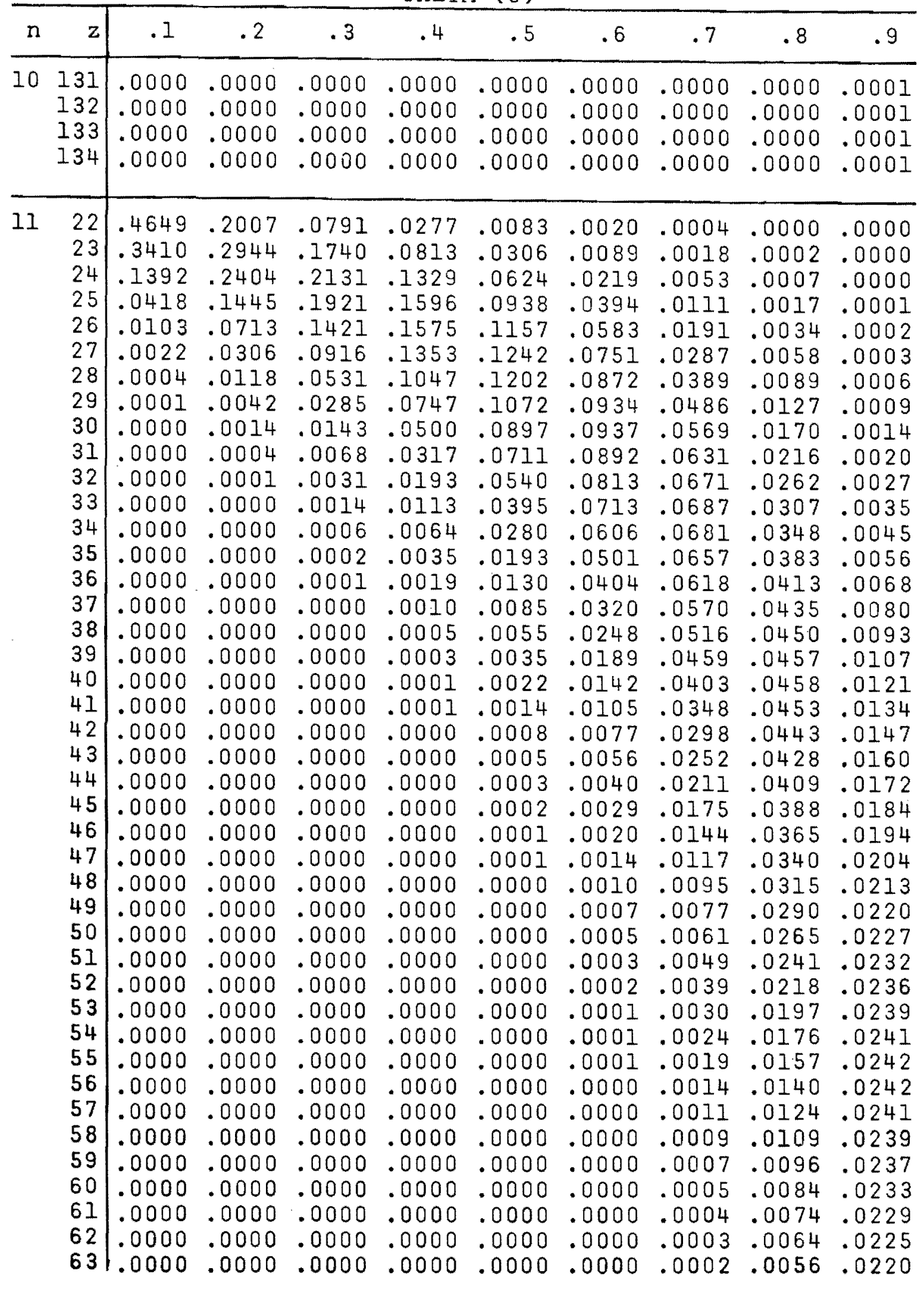


THETA $(\theta)$

\begin{tabular}{|c|c|c|c|c|c|c|c|c|c|c|}
\hline $\mathrm{n}$ & $z$ & .1 & .2 & .3 & .4 & .5 & .6 & .7 & .8 & .9 \\
\hline \multirow[t]{47}{*}{11} & 64 & 000 & 000 & 0000 & 0000 & .0000 & .0000 & .0002 & .0048 & .02. \\
\hline & 65 & .0000 & .0000 & .0000 & .0000 & .0000 & .0000 & .0001 & .0042 & 200 \\
\hline & 66 & .0000 & .0000 & .0000 & .0000 & .0000 & .0000 & .0001 & .0036 & .0202 \\
\hline & 67 & .0000 & .0000 & .0000 & .0000 & .0000 & .0000 & .0000 & & .0196 \\
\hline & 68 & .0000 & .0000 & .0000 & .0000 & .0000 & .0000 & .0000 & .0027 & .0189 \\
\hline & 69 & .0000 & .0000 & .0000 & .0000 & .0000 & .0000 & .0000 & .0023 & .0183 \\
\hline & 70 & .0000 & .0000 & .0000 & .0000 & .0000 & .0000 & .0000 & .0020 & .0176 \\
\hline & 71 & .0000 & .0000 & .0000 & .0000 & .0000 & .0000 & .0000 & .0017 & .0169 \\
\hline & 72 & .0000 & .0000 & .0000 & .0000 & .0000 & .0000 & .0000 & .0014 & . U10 \\
\hline & 73 & .0000 & .0000 & .0000 & .0000 & .0000 & .0000 & .0000 & .0012 & $: 0155$ \\
\hline & 74 & .0000 & .0000 & .0000 & .0000 & .0000 & .0000 & .0000 & .0010 & .0149 \\
\hline & 75 & .0000 & .0000 & .0000 & .0000 & .0000 & .0000 & .0000 & .0009 & .0142 \\
\hline & 76 & .0000 & .0000 & .0000 & .0000 & .0000 & .0000 & .0000 & .0007 & .0135 \\
\hline & 77 & .0000 & .0000 & .0000 & .0000 & .0000 & .0000 & .0000 & .0006 & .0129 \\
\hline & 78 & .0000 & .0000 & .0000 & .0000 & .0000 & .0000 & .0000 & .0005 & .0122 \\
\hline & 79 & .0000 & .0000 & .0000 & .0000 & .0000 & .0000 & .0000 & .0004 & .0116 \\
\hline & 80 & .0000 & .0000 & .0000 & .0000 & .0000 & .0000 & .0000 & .0004 & .0110 \\
\hline & 81 & .0000 & .0000 & .0000 & .0000 & .0000 & .0000 & .0000 & .0003 & .0104 \\
\hline & 82 & .0000 & .0000 & .0000 & .0000 & .0000 & .0000 & .0000 & .0003 & .0099 \\
\hline & 83 & .0000 & .0000 & .0000 & .0000 & .0000 & .0000 & .0000 & .0002 & .0093 \\
\hline & 84 & .0000 & .0000 & .0000 & .0000 & .0000 & .0000 & .00 & .00 & .0088 \\
\hline & 85 & .0000 & .0000 & .0000 & .0000 & .0000 & .0000 & .0000 & .0001 & .0083 \\
\hline & 86 & .0000 & .0000 & .0000 & .0000 & .0000 & .0000 & .0000 & .00 & .0078 \\
\hline & 87 & .0000 & .0000 & .0000 & .0000 & .0000 & .0000 & .0000 & .0001 & .0074 \\
\hline & 88 & .0000 & .0000 & .0000 & .0000 & .0000 & .0000 & .0000 & .0000 & .0069 \\
\hline & 89 & .0000 & .0000 & .0000 & .0000 & .0000 & .0000 & .0000 & .0000 & .0065 \\
\hline & 90 & .0000 & .0000 & .0000 & .0000 & .0000 & .0000 & .0000 & .0000 & .0061 \\
\hline & 91 & .0000 & .0000 & .0000 & .0000 & .0000 & .0000 & .00 & .0000 & .0 \\
\hline & 92 & .0000 & .0000 & .0000 & .0000 & .0000 & .0000 & .0000 & .0000 & .0054 \\
\hline & 93 & .0000 & .0000 & .0000 & .0000 & .00 & & & & \\
\hline & 94 & .0000 & .0000 & .0000 & .0000 & .0000 & .0000 & .0000 & .0000 & .0047 \\
\hline & 95 & & .00 & .0000 & .0000 & .00 & & & & .0 \\
\hline & 96 & .0000 & .0000 & .0000 & .0000 & .0000 & .0000 & .00 & .0000 & .0041 \\
\hline & 7 & .0000 & .0000 & .0000 & .0000 & .0000 & .00 & .0 & .0 & .0039 \\
\hline & 98 & .0000 & .0000 & .0 & .0000 & .00 & .0 & .0 & .0 & 00 \\
\hline & 99 & .0000 & .0000 & .0000 & .0000 & .0000 & .0000 & .0000 & .0000 & .0034 \\
\hline & 100 & .0000 & .0000 & .0000 & .0000 & .00 & .00 & .0 & .0 & \\
\hline & 101 & .0000 & .0000 & .0000 & .0000 & .0000 & .0000 & .0000 & .0000 & .0029 \\
\hline & 102 & .0000 & .0000 & .0000 & .0000 & .0000 & .00 & .00 & .05 & .0027 \\
\hline & 103 & .0000 & .0000 & .0000 & .0000 & .0000 & .0000 & .0000 & .0000 & .0025 \\
\hline & 104 & & .0000 & .0000 & .0000 & .0000 & & .0 & .0000 & \\
\hline & 105 & .0000 & .0000 & .0000 & .0000 & .0000 & .00 & .05 & .0000 & .0022 \\
\hline & 106 & & & 00 & .00 & & & & & .0020 \\
\hline & 107 & .0000 & .0000 & .0000 & .0000 & .0000 & .0000 & .0000 & .0000 & .0019 \\
\hline & 10 & & & .0000 & .0000 & & & & .0000 & .0017 \\
\hline & 10 & & .0000 & .0000 & .0000 & .0 & .0 & & & (1) \\
\hline & & 00 & & 0000 & .0000 & .0000 & 0000 & & 00 & \\
\hline
\end{tabular}


THETA $(\theta)$

\begin{tabular}{|c|c|c|c|c|c|c|c|c|c|c|}
\hline $\mathrm{n}$ & $z$ & .1 & .2 & .3 & .4 & .5 & .6 & .7 & .8 & .9 \\
\hline 1 & $\begin{array}{l}111 \\
112 \\
113 \\
114 \\
115 \\
116 \\
117 \\
118 \\
119 \\
120 \\
121 \\
122 \\
123 \\
124 \\
125 \\
126 \\
127 \\
128 \\
129 \\
130 \\
131 \\
132 \\
133 \\
134 \\
135 \\
136 \\
137 \\
138 \\
139 \\
140 \\
141 \\
142\end{array}$ & $\begin{array}{l}.0000 \\
.0000 \\
.0000 \\
.0000 \\
.0000 \\
.0000 \\
.0000 \\
.0000 \\
.0000 \\
.0000 \\
.0000 \\
.0000 \\
.0000 \\
.0000 \\
.0000 \\
.0000 \\
.0000 \\
.0000 \\
.0000 \\
.0000 \\
.0000 \\
.0000 \\
.0000 \\
.0000 \\
.0000 \\
.0000 \\
.0000 \\
.0000 \\
.0000 \\
.0000 \\
.0000 \\
.0000\end{array}$ & $\begin{array}{l}.0000 \\
.0000 \\
.0000 \\
.0000 \\
.0000 \\
.0000 \\
.0000 \\
.0000 \\
.0000 \\
.0000 \\
.0000 \\
.0000 \\
.0000 \\
.0000 \\
.0000 \\
.0000 \\
.0000 \\
.0000 \\
.0000 \\
.0000 \\
.0000 \\
.0000 \\
.0000 \\
.0000 \\
.0000 \\
.0000 \\
.0000 \\
.0000 \\
.0000 \\
.0000 \\
.0000 \\
.0000\end{array}$ & $\begin{array}{l}.0000 \\
.0000 \\
.0000 \\
.0000 \\
.0000 \\
.0000 \\
.0000 \\
.0000 \\
.0000 \\
.0000 \\
.0000 \\
.0000 \\
.0000 \\
.0000 \\
.0000 \\
.0000 \\
.0000 \\
.0000 \\
.0000 \\
.0000 \\
.0000 \\
.0000 \\
.0000 \\
.0000 \\
.0000 \\
.0000 \\
.0000 \\
.0000 \\
.0000 \\
.0000 \\
.0000 \\
.0000\end{array}$ & $\begin{array}{l}.0000 \\
.0000 \\
.0000 \\
.0000 \\
.0000 \\
.0000 \\
.0000 \\
.0000 \\
.0000 \\
.0000 \\
.0000 \\
.0000 \\
.0000 \\
.0000 \\
.0000 \\
.0000 \\
.0000 \\
.0000 \\
.0000 \\
.0000 \\
.0000 \\
.0000 \\
.0000 \\
.0000 \\
.0000 \\
.0000 \\
.0000 \\
.0000 \\
.0000 \\
.0000 \\
.0000 \\
.0000\end{array}$ & $\begin{array}{l}.0000 \\
.0000 \\
.0000 \\
.0000 \\
.0000 \\
.0000 \\
.0000 \\
.0000 \\
.0000 \\
.0000 \\
.0000 \\
.0000 \\
.0000 \\
.0000 \\
.0000 \\
.0000 \\
.0000 \\
.0000 \\
.0000 \\
.0000 \\
.0000 \\
.0000 \\
.0000 \\
.0000 \\
.0000 \\
.0000 \\
.0000 \\
.0000 \\
.0000 \\
.0000 \\
.0000 \\
.0000\end{array}$ & $\begin{array}{l}.0000 \\
.0000 \\
.0000 \\
.0000 \\
.0000 \\
.0000 \\
.0000 \\
.0000 \\
.0000 \\
.0000 \\
.0000 \\
.0000 \\
.0000 \\
.0000 \\
.0000 \\
.0000 \\
.0000 \\
.0000 \\
.0000 \\
.0000 \\
.0000 \\
.0000 \\
.0000 \\
.0000 \\
.0000 \\
.0000 \\
.0000 \\
.0000 \\
.0000 \\
.0000 \\
.0000 \\
.0000\end{array}$ & $\begin{array}{l}.0000 \\
.0000 \\
.0000 \\
.0000 \\
.0000 \\
.0000 \\
.0000 \\
.0000 \\
.0000 \\
.0000 \\
.0000 \\
.0000 \\
.0000 \\
.0000 \\
.0000 \\
.0000 \\
.0000 \\
.0000 \\
.0000 \\
.0000 \\
.0000 \\
.0000 \\
.0000 \\
.0000 \\
.0000 \\
.0000 \\
.0000 \\
.0000 \\
.0000 \\
.0000 \\
.0000 \\
.0000\end{array}$ & $\begin{array}{l}.0000 \\
.0000 \\
.0000 \\
.0000 \\
.0000 \\
.0000 \\
.0000 \\
.0000 \\
.0000 \\
.0000 \\
.0000 \\
.0000 \\
.0000 \\
.0000 \\
.0000 \\
.0000 \\
.0000 \\
.0000 \\
.0000 \\
.0000 \\
.0000 \\
.0000 \\
.0000 \\
.0000 \\
.0000 \\
.0000 \\
.0000 \\
.0000 \\
.0000 \\
.0000 \\
.0000 \\
.0000\end{array}$ & $\begin{array}{l}.0014 \\
.0013 \\
.0012 \\
.0011 \\
.0010 \\
.0009 \\
.0009 \\
.0008 \\
.0007 \\
.0007 \\
.0006 \\
.0006 \\
.0005 \\
.0005 \\
.0005 \\
.0004 \\
.0004 \\
.0004 \\
.0003 \\
.0003 \\
.0003 \\
.0003 \\
.0002 \\
.0002 \\
.0002 \\
.0002 \\
.0002 \\
.0001 \\
.0001 \\
.0001 \\
.0001 \\
.0001\end{array}$ \\
\hline & $\begin{array}{l}24 \\
25 \\
26 \\
27 \\
28 \\
29 \\
30 \\
31 \\
32 \\
33 \\
34 \\
35 \\
36 \\
37\end{array}$ & $\begin{array}{l}.4337 \\
.3469 \\
.1532 \\
.0494 \\
.0130 \\
.0030 \\
.0006 \\
.0001 \\
.0000 \\
.0000 \\
.0000 \\
.0000 \\
.0000 \\
.0000\end{array}$ & $\begin{array}{l}.2775 \\
.2452 \\
.1582 \\
.0833 \\
.0380 \\
.0155 \\
.0058 \\
.0020 \\
.0007 \\
.0002 \\
.0001 \\
.0000 \\
.0000\end{array}$ & $\begin{array}{l}.1507 \\
.1997 \\
.1932 \\
.1526 \\
.1044 \\
.0640 \\
.0361 \\
.0190 \\
.0094 \\
.0045 \\
.0020 \\
.0009 \\
.0004\end{array}$ & $\begin{array}{l}.1131 \\
.1132 \\
.1460 \\
.1538 \\
.1402 \\
.1147 \\
.0862 \\
.0605 \\
.0401 \\
.0254 \\
.0154 \\
.0091 \\
.0052\end{array}$ & $\begin{array}{l}.0216 \\
.0477 \\
.0769 \\
.1013 \\
.1154 \\
.1180 \\
.1108 \\
.0972 \\
.0806 \\
.0638 \\
.0485 \\
.0356 \\
.0254\end{array}$ & $\begin{array}{l}.0012 \\
.0055 \\
.0147 \\
.0284 \\
.0449 \\
.0614 \\
.0753 \\
.0849 \\
.0893 \\
.0889 \\
.0844 \\
.0770 \\
.0679 \\
.0581\end{array}$ & $\begin{array}{l}.0002 \\
.0010 \\
.0030 \\
.0068 \\
.0126 \\
.0200 \\
.0287 \\
.0377 \\
.0463 \\
.0537 \\
.0595 \\
.0634 \\
.0652 \\
.0651\end{array}$ & $\begin{array}{l}.0000 \\
.0001 \\
.0003 \\
.0009 \\
.0018 \\
.0033 \\
.0053 \\
.0080 \\
.0113 \\
.0150 \\
.0189 \\
.0230 \\
.0271 \\
.0309\end{array}$ & $\begin{array}{l}.0000 \\
.0000 \\
.0001 \\
.0001 \\
.0002 \\
.0004 \\
.0007 \\
.0010 \\
.0014 \\
.0019 \\
.0026 \\
.0033\end{array}$ \\
\hline
\end{tabular}


THETA $(\theta)$

\begin{tabular}{|c|c|c|c|c|c|c|c|c|c|c|}
\hline$n$ & $z$ & .1 & .2 & .3 & .4 & .5 & .6 & .7 & .8 & .9 \\
\hline \multirow[t]{47}{*}{12} & 38 & .0000 & .0000 & .00 & .0029 & .0177 & .0485 & .0634 & .0344 & .0041 \\
\hline & 39 & .0000 & & 01 & & .0120 & .0395 & .0603 & .0374 & 0050 \\
\hline & 40 & .0000 & .0000 & .0000 & .0008 & .0080 & .0316 & .0562 & .0398 & .0060 \\
\hline & 41 & .00 .00 & .0000 & .0000 & .0004 & .0052 & .0248 & .0514 & .0417 & .0071 \\
\hline & 42 & .0000 & .0000 & .0000 & .0002 & .0034 & .0191 & .0463 & .0429 & .0082 \\
\hline & 43 & .0000 & .0000 & .0000 & .0001 & .0021 & .0146 & .0411 & .0435 & .0094 \\
\hline & 44 & .0000 & .0000 & .0000 & .0001 & .007 & .0109 & .0360 & .0436 & .0106 \\
\hline & 45 & .0000 & .0000 & .0000 & .0000 & .0008 & .0081 & .0312 & .0431 & .0118 \\
\hline & 46 & .0000 & .0000 & .0000 & .0000 & .0005 & .0060 & .0267 & .0422 & .0130 \\
\hline & 47 & .0000 & .0000 & .0000 & .0000 & .0003 & .0043 & .0226 & .0409 & .0141 \\
\hline & 48 & .0000 & .0000 & .0000 & .0000 & .0002 & .0031 & .0190 & .0392 & .0153 \\
\hline & 49 & .0000 & .0000 & .0000 & .0000 & .0001 & .0 & .0158 & .0373 & .0163 \\
\hline & 50 & .0000 & .0000 & .0000 & . & .00 & & .0 & .03 & 174 \\
\hline & 51 & .0000 & .0 & .0 & .0 & .00 & & & .0330 & .0183 \\
\hline & 52 & .0000 & .0000 & .0000 & .0000 & .0000 & .0008 & .0087 & .0308 & .0192 \\
\hline & 53 & .0000 & .0000 & .0000 & .0000 & .0000 & .0005 & .0071 & .0285 & .0200 \\
\hline & 54 & .0000 & .0000 & .0000 & .0000 & .0000 & .0004 & .0057 & .0262 & .0207 \\
\hline & 55 & .0000 & .0000 & .0000 & .0000 & .00 & 3 & .0 & .02 & .021 \\
\hline & 56 & .0000 & .0000 & .0000 & .0 & .00 & & .0 & .02 & .0218 \\
\hline & 57 & .0000 & .0 & .0 & .0 & .00 & & .0029 & .01 & .0222 \\
\hline & 58 & .0 & .0 & .0 & .0 & .00 & 01 & .0023 & .0178 & .0225 \\
\hline & 59 & .0000 & .0000 & .0000 & .0000 & .0000 & .0001 & .0018 & .0160 & .0228 \\
\hline & 60 & .0000 & .0000 & .0000 & .0000 & .0000 & .0000 & .0014 & .0143 & .0229 \\
\hline & 61 & .0000 & .0000 & .0000 & .00 & .0000 & 00 & .0011 & .01 & .0230 \\
\hline & 62 & .0000 & .0000 & .0000 & .0 & .00 & & 08 & .0 & .0229 \\
\hline & 63 & .0000 & .0000 & .0 & .0 & .00 & & .0 & .07 & .0228 \\
\hline & 64 & .0000 & .0 & .0 & .0 & .00 & 00 & .0005 & .00 & .0227 \\
\hline & 65 & .0000 & .0000 & .0000 & .0000 & .0000 & 00 & .0004 & .0078 & .0224 \\
\hline & 66 & .0000 & .0000 & .0000 & .0000 & .0000 & .0000 & .0003 & .0068 & .0221 \\
\hline & 67 & .0000 & .0000 & .0000 & .0000 & .00 & .0 & .0 & .0 & .0218 \\
\hline & 68 & .0000 & .0000 & .0000 & .0 & .00 & 0 & 02 & .0 & .0214 \\
\hline & 69 & .0000 & .0000 & .0000 & .0 & .00 & & & & .0209 \\
\hline & 70 & .0000 & .0000 & .0 & & .00 &. & 01 & .0 & .0204 \\
\hline & 71 & .0000 & & & & .0 & & 00 & & .0199 \\
\hline & 72 & & & & .0 & .00 & 00 & .0000 & .0 & .0194 \\
\hline & 73 & .0000 & .0000 & .0000 & .0000 & .0000 & .0000 & .0000 & .0025 & .0188 \\
\hline & 74 & .0000 & .0000 & .0000 & .0000 & .0000 & 00 & .0000 & .00 & .0182 \\
\hline & 75 & .0000 & .0000 & .0000 & .0000 & .0000 & .0000 & .0000 & .00 & .0176 \\
\hline & 76 & .0000 & .0000 & .0000 & .0 & .00 & 00 & .0000 & .0 & .0170 \\
\hline & 77 & .0000 & 00 & & .0 & .00 & & .0 & & .0164 \\
\hline & 78 & & & & & & & .0000 & .0012 & .0157 \\
\hline & 75 & & & .0 & .0000 & .0000 & .0000 & .0000 & .0010 & .0151 \\
\hline & 80 & 10 & .0000 & .0 & .00 & .00 & 00 & .0000 & .0009 & .014 \\
\hline & 8. & 000 & 00 & .0 & .00 & .00 & & .0000 & .00 & .013 \\
\hline & 8 & .0000 & .0000 & .0000 & .0000 & .000 & .0000 & .0000 & .0006 & .0133 \\
\hline & 8 & .0000 & .0000 & .0000 & .00 & .0000 & .0000 & .0000 & .0005 & .0127 \\
\hline & & .0000 & .0000 & .0000 & .0000 & .0000 & .0000 & .0000 & .0004 & .0121 \\
\hline
\end{tabular}


THETA $(\theta)$

\begin{tabular}{|c|c|c|c|c|c|c|c|c|c|c|}
\hline $\mathrm{n}$ & $z$ & .1 & .2 & .3 & .4 & .5 & .6 & .7 & .8 & .9 \\
\hline \multirow[t]{47}{*}{12} & 85 & .0000 & .0000 & .0000 & .0000 & .0000 & .0000 & .0000 & .0004 & .0115 \\
\hline & 86 & .0000 & .0000 & .0000 & .0000 & .0000 & .0000 & .0000 & .0003 & .0110 \\
\hline & 87 & .0000 & .0000 & .0000 & .0000 & .0000 & .0000 & .0000 & .0003 & .0104 \\
\hline & 88 & .0000 & .0000 & .0000 & .0000 & .0000 & .0000 & .0000 & .0002 & .0099 \\
\hline & 89 & .0000 & .0000 & .0000 & .0000 & .0000 & .0000 & .0000 & .0002 & .0094 \\
\hline & 90 & .0000 & .0000 & .0000 & .0000 & .0000 & .0000 & .0000 & .0001 & .0089 \\
\hline & 91 & .0000 & .0000 & .0000 & .0000 & .0000 & .0000 & .0000 & .0001 & .0084 \\
\hline & 92 & .0000 & .0000 & .0000 & .0000 & .0000 & .0000 & .0000 & .0001 & .0079 \\
\hline & 93 & .0000 & .0000 & .0000 & .0000 & .0000 & .0000 & .0000 & .0000 & .0075 \\
\hline & 94 & .0000 & .0000 & .0000 & .0000 & .0000 & .0000 & .0000 & .0000 & .0071 \\
\hline & 95 & .0000 & .0000 & .0000 & .0000 & .0000 & .0000 & .0000 & .0000 & .0067 \\
\hline & 96 & .0000 & .0000 & .0000 & .0000 & .0000 & .0000 & .0000 & .0000 & .0063 \\
\hline & 97 & .0000 & .0000 & .0000 & .0000 & .0000 & .0000 & .0000 & .0000 & .0059 \\
\hline & 98 & .0000 & .0000 & .0000 & .0000 & .0000 & .0000 & .0000 & .0000 & .0055 \\
\hline & 99 & .0000 & .0000 & .0000 & .0000 & .0000 & .0000 & .0000 & .0000 & .0052 \\
\hline & 100 & .0000 & .0000 & .0000 & .0000 & .0000 & .0000 & .0000 & .0000 & .0049 \\
\hline & 101 & .0000 & .0000 & .0000 & .0000 & .0000 & .0000 & .0000 & .0000 & .0046 \\
\hline & 102 & .0000 & .0000 & .0000 & .0000 & .0000 & .0000 & .0000 & .0000 & .0043 \\
\hline & 103 & .0000 & .0000 & .0000 & .0000 & .0000 & .0000 & .0000 & .0000 & .0040 \\
\hline & 104 & .0000 & .0000 & .0000 & .0000 & .0000 & .0000 & .0000 & .0000 & .0038 \\
\hline & 105 & .0000 & .0000 & .0000 & .0000 & .0000 & .0000 & .0000 & .0000 & .0035 \\
\hline & 106 & .0000 & .0000 & .0000 & .0000 & .0000 & .0000 & .0000 & .0000 & .0033 \\
\hline & 107 & .0000 & .0000 & .0000 & .0000 & .0000 & .0000 & .0000 & .0000 & .0031 \\
\hline & 108 & .0000 & .0000 & .0000 & .0000 & .0000 & .0000 & .0000 & .0000 & .0029 \\
\hline & 109 & .0000 & .0000 & .0000 & .0000 & .0000 & .0000 & .0000 & .0000 & .0027 \\
\hline & 110 & .0000 & .0000 & .0000 & .0000 & .0000 & .0000 & .0000 & .0000 & .0025 \\
\hline & 111 & .0000 & .0000 & .0000 & .0000 & .0000 & .0000 & .0000 & .0000 & .0023 \\
\hline & 112 & .0000 & .0000 & .0000 & .0000 & .0000 & .0000 & .0000 & .0000 & .0022 \\
\hline & 113 & .0000 & .0000 & .0000 & .0000 & .0000 & .0000 & .0000 & .0000 & .0020 \\
\hline & 114 & .0000 & .0000 & .0000 & .0000 & .0000 & .0000 & .0000 & .0000 & .0019 \\
\hline & 115 & .0000 & .0000 & .0000 & .0000 & .0000 & .0000 & .0000 & .0000 & .0018 \\
\hline & 116 & .0000 & .0000 & .0000 & .0000 & .0000 & .0000 & .0000 & .0000 & .0016 \\
\hline & 117 & .0000 & .0000 & .0000 & .0000 & .0000 & .0000 & .0000 & .0000 & .0015 \\
\hline & 118 & .0000 & .0000 & .0000 & .0000 & .0000 & .0000 & .0000 & .0000 & .0014 \\
\hline & 119 & .0000 & .0000 & .0000 & .0000 & .0000 & .0000 & .0000 & .0000 & .0013 \\
\hline & 120 & .0000 & .0000 & .0000 & .0000 & .0000 & .0000 & .0000 & .0000 & .0012 \\
\hline & 121 & .0000 & .0000 & .0000 & .0000 & .0000 & .0000 & .0000 & .0000 & .0011 \\
\hline & 122 & .0000 & .0000 & .0000 & .0000 & .0000 & .0000 & .0000 & .0000 & .0010 \\
\hline & 123 & .0000 & .0000 & .0000 & .0000 & .0000 & .0000 & .0000 & .0000 & .0010 \\
\hline & 124 & .0000 & .0000 & .0000 & .0000 & .0000 & .0000 & .0000 & .0000 & .0009 \\
\hline & 125 & .0000 & .0000 & .0000 & .0000 & .0000 & .0000 & .0000 & .0000 & .0008 \\
\hline & 126 & .0000 & .0000 & .0000 & .0000 & .0000 & .0000 & .0000 & .0000 & .0008 \\
\hline & 127 & .0000 & .0000 & .0000 & .0000 & .0000 & .0000 & .0000 & .0000 & .0007 \\
\hline & 120 & .0000 & .0000 & .0000 & .0000 & .0000 & .0000 & .0000 & .0000 & .0007 \\
\hline & 129 & .0000 & .0000 & .0000 & .0000 & .0000 & .0000 & .0000 & .0000 & .0006 \\
\hline & 30 & .0000 & .0000 & .0000 & .0000 & .0000 & .0000 & .0000 & .0000 & .0005 \\
\hline & 131 & .0000 & .0000 & .0000 & .0000 & .0000 & .0000 & .0000 & .0000 & .0005 \\
\hline
\end{tabular}


THETA $(\theta)$

\begin{tabular}{|c|c|c|c|c|c|c|c|c|c|c|}
\hline$n$ & $\mathbf{z}$ & .1 & .2 & .3 & .4 & .5 & .6 & .7 & .8 & .9 \\
\hline & $\begin{array}{l}132 \\
133 \\
134 \\
135 \\
136 \\
137 \\
138 \\
139 \\
140 \\
141 \\
142 \\
143 \\
144 \\
145 \\
146 \\
147 \\
148 \\
149 \\
150\end{array}$ & $\begin{array}{l}.0000 \\
.0000 \\
.0000 \\
.0000 \\
.0000 \\
.0000 \\
.0000 \\
.0000 \\
.0000 \\
.0000 \\
.0000 \\
.0000 \\
.0000 \\
.0000 \\
.0000 \\
.0000 \\
.0000 \\
.0000 \\
.0000\end{array}$ & $\begin{array}{l}.0000 \\
.0000 \\
.0000 \\
.0000 \\
.0000 \\
.0000 \\
.0000 \\
.0000 \\
.0000 \\
.0000 \\
.0000 \\
.0000 \\
.0000 \\
.0000 \\
.0000 \\
.0000 \\
.0000 \\
.0000 \\
.0000\end{array}$ & $\begin{array}{l}.0000 \\
.0000 \\
.0000 \\
.0000 \\
.0000 \\
.0000 \\
.0000 \\
.0000 \\
.0000 \\
.0000 \\
.0000 \\
.0000 \\
.0000 \\
.0000 \\
.0000 \\
.0000 \\
.0000 \\
.0000 \\
.0000\end{array}$ & $\begin{array}{l}.0000 \\
.0000 \\
.0000 \\
.0000 \\
.0000 \\
.0000 \\
.0000 \\
.0000 \\
.0000 \\
.0000 \\
.0000 \\
.0000 \\
.0000 \\
.0000 \\
.0000 \\
.0000 \\
.0000 \\
.0000 \\
.0000\end{array}$ & $\begin{array}{l}.0000 \\
.0000 \\
.0000 \\
.0000 \\
.0000 \\
.0000 \\
.0000 \\
.0000 \\
.0000 \\
.0000 \\
.0000 \\
.0000 \\
.0000 \\
.0000 \\
.0000 \\
.0000 \\
.0000 \\
.0000 \\
.0000\end{array}$ & $\begin{array}{l}.0000 \\
.0000 \\
.0000 \\
.0000 \\
.0000 \\
.0000 \\
.0000 \\
.0000 \\
.0000 \\
.0000 \\
.0000 \\
.0000 \\
.0000 \\
.0000 \\
.0000 \\
.0000 \\
.0000 \\
.0000 \\
.0000\end{array}$ & $\begin{array}{l}.0000 \\
.0000 \\
.0000 \\
.0000 \\
.0000 \\
.0000 \\
.0000 \\
.0000 \\
.0000 \\
.0000 \\
.0000 \\
.0000 \\
.0000 \\
.0000 \\
.0000 \\
.0000 \\
.0000 \\
.0000 \\
.0000\end{array}$ & $\begin{array}{l}0 \\
0 \\
0 \\
0 \\
0 \\
0 \\
0 \\
0 \\
0 \\
0 \\
0 \\
0 \\
0 \\
0 \\
0 \\
0 \\
0\end{array}$ & $\begin{array}{l}.0005 \\
.0004 \\
.0004 \\
.0004 \\
.0003 \\
.0003 \\
.0003 \\
.0003 \\
.0003 \\
.0002 \\
.0002 \\
.0002 \\
.0002 \\
.0002 \\
.0001 \\
.0001 \\
.0001 \\
.0001 \\
.0001\end{array}$ \\
\hline & $\begin{array}{l}26 \\
27 \\
28 \\
29 \\
30 \\
31 \\
32 \\
33 \\
34 \\
35 \\
36 \\
37 \\
38 \\
39 \\
40 \\
41 \\
42 \\
43 \\
44 \\
45 \\
46 \\
47 \\
48 \\
49 \\
50 \\
51 \\
52\end{array}$ & $\begin{array}{l}.0574 \\
.0161 \\
.0039 \\
.0008 \\
.0002 \\
.0000 \\
.0000 \\
.0000 \\
.0000 \\
.0000 \\
.0000 \\
.0000 \\
.0000 \\
.0000 \\
.0000 \\
.0000 \\
.0000 \\
.0000 \\
.0000 \\
.0000 \\
.0000 \\
.0000 \\
.0000 \\
.0000\end{array}$ & $\begin{array}{l}.3598 \\
.2468 \\
.1702 \\
.0953 \\
.0460 \\
.0198 \\
.0078 \\
.0029 \\
.0010 \\
.0003 \\
.0001 \\
.0000 \\
.0000 \\
.0000 \\
.0000 \\
.0000 \\
.0000 \\
.0000 \\
.0000 \\
.0000 \\
.0000 \\
.0000 \\
.0000 \\
.0000 \\
.0000 \\
.0000\end{array}$ & $\begin{array}{l}.1296 \\
.1847 \\
.1911 \\
.1604 \\
.1161 \\
.0750 \\
.0444 \\
.0245 \\
.0127 \\
.0063 \\
.0030 \\
.0014 \\
.0006 \\
.0003 \\
.0001 \\
.0000 \\
.0000 \\
.0000 \\
.0000 \\
.0000 \\
.0000 \\
.0000 \\
.0000 \\
.0000 \\
.0000 \\
.0000\end{array}$ & $\begin{array}{l}.0501 \\
.0952 \\
.1313 \\
.1470 \\
.1418 \\
.1222 \\
.0964 \\
.0708 \\
.0490 \\
.0323 \\
.0204 \\
.0124 \\
.0073 \\
.0042 \\
.0024 \\
.0013 \\
.0007 \\
.0004 \\
.0002 \\
.0001 \\
.0000 \\
.0000 \\
.0000 \\
.0000 \\
.0000 \\
.0000\end{array}$ & $\begin{array}{l}.0151 \\
.0360 \\
.0620 \\
.0868 \\
.1046 \\
.1127 \\
.1112 \\
.1020 \\
.0883 \\
.0727 \\
.0574 \\
.0437 \\
.0323 \\
.0232 \\
.0162 \\
.0111 \\
.0075 \\
.0049 \\
.0032 \\
.0021 \\
.0013 \\
.0008 \\
.0005 \\
.0003 \\
.0002 \\
.0001\end{array}$ & $\begin{array}{l}.0007 \\
.0034 \\
.0097 \\
.0201 \\
.0338 \\
.0489 \\
.0633 \\
.0749 \\
.0825 \\
.0857 \\
.0847 \\
.0802 \\
.0733 \\
.0649 \\
.0559 \\
.0470 \\
.0387 \\
.0312 \\
.0247 \\
.0193 \\
.0148 \\
.0113 \\
.0085 \\
.0063 \\
.0046 \\
.0034 \\
.0024\end{array}$ & $\begin{array}{l}.0017 \\
.0041 \\
.0081 \\
.0136 \\
.0206 \\
.0284 \\
.0365 \\
.0442 \\
.0510 \\
.0564 \\
.0601 \\
.0621 \\
.0624 \\
.0612 \\
.0587 \\
.0553 \\
.0511 \\
.0465 \\
.0418 \\
.0370 \\
.0324 \\
.0281 \\
.0241 \\
.0204 \\
.0172\end{array}$ & $\begin{array}{l}.0000 \\
.0002 \\
.0004 \\
.0009 \\
.0018 \\
.0031 \\
.0049 \\
.0072 \\
.0100 \\
.0132 \\
.0167 \\
.0203 \\
.0240 \\
.0275 \\
.0309 \\
.0338 \\
.0364 \\
.0385 \\
.0400 \\
.0411 \\
.0416 \\
.0416 \\
.0412 \\
.0403 \\
.0392 \\
.0377\end{array}$ & $\begin{array}{l}.0000 \\
.0000 \\
.0000 \\
.0000 \\
.0000 \\
.0001 \\
.0001 \\
.0002 \\
.0003 \\
.0005 \\
.0007 \\
.0010 \\
.0014 \\
.0019 \\
.0024 \\
.0030 \\
.0038 \\
.0045 \\
.0054 \\
.0063 \\
.0073 \\
.0083 \\
.0093 \\
.0104 \\
.0115 \\
.0125 \\
.0136\end{array}$ \\
\hline
\end{tabular}


THETA $(\theta)$

\begin{tabular}{|c|c|c|c|c|c|c|c|c|c|c|}
\hline $\mathrm{n}$ & $z$ & .1 & .2 & .3 & .4 & .5 & .6 & .7 & .8 & .9 \\
\hline \multirow[t]{47}{*}{13} & 53 & .0000 & .0000 & .00 & .0000 & & & & & \\
\hline & 54 & & & & & & & & & \\
\hline & 55 & .0000 & .0000 & .0000 & .0000 & .0000 & .0009 & .0098 & .0321 & .0166 \\
\hline & 56 & .0000 & .0000 & .0000 & .0000 & .0000 & .0006 & .0080 & .0300 & .0173 \\
\hline & 57 & .0000 & .0000 & .0000 & .0000 & .0000 & .0004 & .0065 & .0279 & \\
\hline & 58 & .0000 & .0000 & .0000 & .0000 & .0000 & .0003 & .0053 & .0258 & .0188 \\
\hline & 59 & .0000 & .0000 & .0000 & .0000 & .0000 & .0002 & .0043 & .0238 & .0195 \\
\hline & 60 & .0000 & .0000 & .0000 & .0000 & .0000 & .0001 & .0034 & .0217 & .0201 \\
\hline & 6]. & .0000 & .0000 & .0000 & .0000 & .0000 & .0001 & .0027 & .0198 & .0206 \\
\hline & 62 & .0000 & .0000 & .0000 & .0000 & .0000 & .0000 & .0022 & .0180 & .0210 \\
\hline & 63 & .0000 & .0000 & .0000 & .0000 & .0000 & .0000 & .0017 & .0162 & .0213 \\
\hline & 64 & .0000 & .0000 & .0000 & .0000 & .0000 & .0000 & .0013 & .0146 & .0216 \\
\hline & 65 & .0000 & .0000 & .0000 & .0000 & .0000 & .0000 & .0011 & .0131 & .0218 \\
\hline & 66 & .0000 & .0000 & .0000 & .0000 & .0000 & .0000 & .0008 & .0117 & .0219 \\
\hline & 67 & .0000 & .0000 & .0000 & .0000 & .0000 & .0000 & .0006 & .0104 & .0219 \\
\hline & 68 & .0000 & .0000 & .0000 & .0000 & .0000 & .0000 & .0005 & .0092 & .0219 \\
\hline & 69 & .0000 & .0000 & .0000 & .0000 & .0000 & .0000 & .0004 & .0082 & .0218 \\
\hline & 70 & .0000 & .0000 & .0000 & .0000 & .0000 & .0000 & .0003 & .0072 & .027 \\
\hline & 71 & .0000 & .0000 & .0000 & .0000 & .0000 & .0000 & .0002 & .0063 & .0214 \\
\hline & 72 & .0000 & .0000 & 0000 & .0000 & .0000 & .0000 & .0002 & .0056 & .0211 \\
\hline & 73 & .0000 & .0000 & .0000 & .0000 & .0000 & .0000 & .0001 & .0049 & .0208 \\
\hline & 74 & .0000 & .0000 & .0000 & .0000 & .0000 & .0000 & .0001 & .0043 & .0204 \\
\hline & 75 & .0000 & .0000 & .0000 & .0000 & .0000 & .0000 & .0000 & .0037 & .0200 \\
\hline & 76 & .0000 & .0000 & .0000 & .0000 & .0000 & .0000 & .0000 & .0032 & .0196 \\
\hline & 77 & 1000 & .0000 & .00 & & 00 & & & & \\
\hline & 78 & .0000 & .0000 & .0000 & .0000 & .0000 & .0000 & .0000 & .0024 & .0186 \\
\hline & 79 & & & & & & & & & \\
\hline & 80 & .0000 & .0000 & .0000 & .0000 & 0000 & .0000 & .0000 & .0018 & .0176 \\
\hline & 8 & & & & & .0000 & & & & \\
\hline & 82 & .0000 & .0000 & .0000 & .0000 & .0000 & .0000 & .0000 & .0013 & .0164 \\
\hline & 8 & & & & & .0000 & .0 & & & \\
\hline & 84 & .0000 & .0000 & .0000 & .0000 & .0000 & .0000 & .0000 & .0010 & .0153 \\
\hline & 85 & .0000 & .0000 & .0000 & .0 & .0000 & .0 & .0 & & .011 \\
\hline & 86 & .0000 & .0 & .0 & .0 & .0000 & .00 & .0 & .0 & .0142 \\
\hline & 87 & .0000 & .0000 & .0000 & .0000 & .0000 & .0000 & .0000 & .0006 & .0136 \\
\hline & 88 & & .0000 & .00 & .00 & .0000 & & & & \\
\hline & 89 & .0000 & .0000 & .0000 & .0000 & .0000 & .0000 & .0000 & .0004 & .0125 \\
\hline & 90 & & .0000 & .0000 & .0000 & .0000 & & & .0004 & \\
\hline & 91 & .00 & .0000 & .0000 & .0000 & .0000 & .0000 & .0000 & .0003 & .0114 \\
\hline & 92 & 00 & .0000 & .06 & .0000 & .0000 & & & .00 & סח דת \\
\hline & 93 & .0000 & .0000 & .0000 & .0000 & .0000 & .0000 & .0000 & .0002 & .0103 \\
\hline & 94 & 10 & 0000 & .0000 & .0000 & .0000 & & & .0002 & .0098 \\
\hline & 95 & .0000 & .0000 & .0000 & .0000 & .0000 & .0000 & .0000 & .0001 & .0094 \\
\hline & 96 & .0000 & .0000 & .0000 & .0000 & .0000 & .0000 & .0000 & .0001 & .0089 \\
\hline & 97 & & .0000 & .0000 & .0000 & .0000 & .0000 & .0000 & .0001 & \\
\hline & & & & .0000 & .0000 & .0000 & .0000 & .0000 & .0000 & .0080 \\
\hline & 991 & .0000 & .0000 & .0000 & .0000 & .0000 & .0000 & .0000 & .0000 & .0076 \\
\hline
\end{tabular}


THETA $(\theta)$

\begin{tabular}{|c|c|c|c|c|c|c|c|c|c|c|}
\hline$n$ & $z$ & .1 & .2 & .3 & .4 & .5 & .6 & .7 & .8 & .9 \\
\hline \multirow[t]{47}{*}{13} & 100 & .0000 & .0000 & 00 & .0000 & .0000 & .0000 & .0000 & .0000 & .0072 \\
\hline & 101 & .0000 & .0000 & . O & & .0000 & .00 & .0000 & .0000 & .0068 \\
\hline & 102 & .0000 & .0000 & .0000 & .00 & .0000 & .00 & .0000 & .0000 & 0064 \\
\hline & 103 & .0000 & .0000 & 00 & .0000 & .0000 & .00 & .0000 & .0000 & 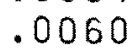 \\
\hline & 104 & .0000 & .0000 & .0000 & .0000 & .0000 & .0000 & .0000 & .0000 & 005 \\
\hline & 105 & .0000 & .0000 & .0000 & .0000 & .0000 & .0000 & .0000 & .0000 & .00 \\
\hline & 106 & .0000 & .0000 & .0000 & .0000 & .0000 & .0 & .00 & .0000 & . \\
\hline & 107 & .0000 & .0000 & .0000 & & & & .00 & 00 & \\
\hline & 108 & .0000 & .00 & .00 & .0 & .0000 & .00 & .00 & .0000 & 00 \\
\hline & 109 & .0000 & .00 & .0000 & .00 & .0000 & .00 & .00 & .0000 & .0042 \\
\hline & 110 & .0000 & .0000 & .0000 & .0000 & .0000 & .00 & .00 & .0000 & .0039 \\
\hline & 111 & .0000 & .0000 & .0000 & .0000 & .0000 & .0000 & .00 & .0000 & .0037 \\
\hline & 112 & .0000 & .0000 & .0000 & .0000 & .0000 & 0 & .00 & .0000 & \\
\hline & 113 & .0000 & .0000 & .0000 & .0 & .00 & & .00 & 00 & 32 \\
\hline & 114 & .0000 & .0 & .00 & .0 & .00 & • & .00 & .0000 & .0030 \\
\hline & 115 & .0 & .0 & .00 & .00 & .0000 & .00 & .00 & .0000 & .0028 \\
\hline & 116 & .0000 & .0000 & .0000 & .0000 & .0000 & .00 & .00 & .0000 & .0027 \\
\hline & 117 & .0000 & .0000 & .0000 & .0000 & .0000 & .00 & .00 & 00 & .00 \\
\hline & 118 & .0000 & .0000 & .0000 & .0 & .0000 & .00 &. & 00 & .002 \\
\hline & 119 & .0000 & .0000 & .0000 & .0 & .00 & & & & 22 \\
\hline & 120 & .0000 & 0 & .00 & & .0 & & & & .0020 \\
\hline & 121 & .0000 & .0 & .00 & & .00 & & .00 & .0000 & .0019 \\
\hline & 122 & .0 & .0 & .0 & .0 & .0000 & .00 & .00 & .0000 & .0018 \\
\hline & 123 & .0000 & .0000 & .0000 & .00 & .0000 & .00 & .00 & .0000 & .0016 \\
\hline & 124 & .0000 & .0000 & .0000 & .00 & .0000 & 0 & .0 & 00 & 0 \\
\hline & 125 & 0000 & .0 & .00 & .0 & .0000 & & & & \\
\hline & 126 & .0000 & .0 & .00 & & .00 & & .0 & 0 & \\
\hline & 127 & .0000 & .0 & .00 & & .0 & & & & 12 \\
\hline & 128 & .00 & .0 & .0 & & .00 & & & 00 & .0011 \\
\hline & 129 & & & & & .0000 & & .00 & .0000 & .0011 \\
\hline & 130 & .0000 & .0000 & .0000 & .0 & .0000 & .0 & .00 & .0000 & .0010 \\
\hline & 131 & .0000 & .0000 & .0000 & .00 & .0000 & & & & .0 \\
\hline & 2 & 0000 & ח & .00 & & .00 & & & & \\
\hline & 3 & .0000 & .0 & .0 & & .00 & & & & \\
\hline & 34 & .0000 & .0 & .0 & & 0 & & .0 & 0 & 07 \\
\hline & 135 & & & & & $:$ & & & & \\
\hline & 136 & & & & & & & & 0 & .0 \\
\hline & 137 & .0000 & .0 & .00 & & .0000 & & & .00 & .0006 \\
\hline & 138 & .0000 & .0000 & .0000 & .0 & .0000 & .0 & .0 & .0000 & .0005 \\
\hline & 139 & .0000 & .0000 & .0000 & .0 & .00 & & .0 & .0 & \\
\hline & & 000 & & & & .00 & & & & \\
\hline & & .0000 & .0 & .00 & .0 & .00 & & .0 & 10 & .0004 \\
\hline & 142 & & & & & & & & .0000 & .0004 \\
\hline & 143 & .0000 & & .0000 & .0000 & .0000 & .0000 & .0000 & .0000 & .0004 \\
\hline & 144 & .0000 & .0000 & .0000 & .0000 & .0000 & .0000 & .00 & .0000 & .0003 \\
\hline & 145 & .0000 & 00 & .0000 & .00 & .00 & & .0 & & \\
\hline & & & & & & 0 & & & .0000 & \\
\hline
\end{tabular}


THETA $(\theta)$

\begin{tabular}{|c|c|c|c|c|c|c|c|c|c|c|}
\hline $\mathrm{n}$ & $z$ & .1 & .2 & .3 & .4 & .5 & .6 & .7 & .8 & 9 \\
\hline 3 & $\begin{array}{l}147 \\
148 \\
149 \\
150 \\
151 \\
152 \\
153 \\
154 \\
155 \\
156 \\
157\end{array}$ & $\begin{array}{l}.0000 \\
.0000 \\
.0000 \\
.0000 \\
.0000 \\
.0000 \\
.0000 \\
.0000 \\
.0000 \\
.0000 \\
.0000\end{array}$ & $\begin{array}{l}.0000 \\
.0000 \\
.0000 \\
.0000 \\
.0000 \\
.0000 \\
.0000 \\
.0000 \\
.0000 \\
.0000 \\
.0000\end{array}$ & $\begin{array}{l}.0000 \\
.0000 \\
.0000 \\
.0000 \\
.0000 \\
.0000 \\
.0000 \\
.0000 \\
.0000 \\
.0000 \\
.0000\end{array}$ & $\begin{array}{l}.0000 \\
.0000 \\
.0000 \\
.0000 \\
.0000 \\
.0000 \\
.0000 \\
.0000 \\
.0000 \\
.0000 \\
.0000\end{array}$ & $\begin{array}{l}.0000 \\
.0000 \\
.0000 \\
.0000 \\
.0000 \\
.0000 \\
.0000 \\
.0000 \\
.0000 \\
.0000 \\
.0000\end{array}$ & $\begin{array}{l}.0000 \\
.0000 \\
.0000 \\
.0000 \\
.0000 \\
.0000 \\
.0000 \\
.0000 \\
.0000 \\
.0000 \\
.0000\end{array}$ & $\begin{array}{l}.0000 \\
.0000 \\
.0000 \\
.0000 \\
.0000 \\
.0000 \\
.0000 \\
.0000 \\
.0000 \\
.0000 \\
.0000\end{array}$ & $\begin{array}{l}.0000 \\
.0000 \\
.0000 \\
.0000 \\
.0000 \\
.0000 \\
.0000 \\
.0000 \\
.0000 \\
.0000 \\
.0000\end{array}$ & $\begin{array}{r}.0003 \\
.0002 \\
.0002 \\
.0002 \\
.0002 \\
.0002 \\
.0002 \\
.0001 \\
.0001 \\
.0001 \\
.0001\end{array}$ \\
\hline & $\begin{array}{l}28 \\
29 \\
30 \\
31 \\
32 \\
33 \\
34 \\
35 \\
36 \\
37 \\
38 \\
39 \\
40 \\
41 \\
42 \\
43 \\
44 \\
45 \\
46 \\
47 \\
48 \\
49 \\
50 \\
51 \\
52 \\
53 \\
54 \\
55 \\
56 \\
57 \\
58 \\
59 \\
60 \\
61 \\
62\end{array}$ & $\begin{array}{l}.3773 \\
.3521 \\
.1790 \\
.0657 \\
.0195 \\
.0050 \\
.0011 \\
.0002 \\
.0000 \\
.0000 \\
.0000 \\
.0000 \\
.0000 \\
.0000 \\
.0000 \\
.0000 \\
.0000 \\
.0000 \\
.0000 \\
.0000 \\
.0000 \\
.0000 \\
.0000 \\
.0000 \\
.0000 \\
.0000 \\
.0000 \\
.0000 \\
.0000 \\
.0000 \\
.0000 \\
.0000 \\
.0000 \\
.0000 \\
.0000\end{array}$ & $\begin{array}{l}.1295 \\
.2418 \\
.2458 \\
.1804 \\
.1070 \\
.0545 \\
.0247 \\
.0102 \\
.0039 \\
.0014 \\
.0005 \\
.0002 \\
.0000 \\
.0000 \\
.0000 \\
.0000 \\
.0000 \\
.0000 \\
.0000 \\
.0000 \\
.0000 \\
.0000 \\
.0000 \\
.0000 \\
.0000 \\
.0000 \\
.0000 \\
.0000 \\
.0000 \\
.0000 \\
.0000 \\
.0000 \\
.0000 \\
.0000 \\
.0000\end{array}$ & $\begin{array}{l}.0396 \\
.1108 \\
.1690 \\
.1861 \\
.1655 \\
.1264 \\
.0859 \\
.0532 \\
.0306 \\
.0166 \\
.0085 \\
.0042 \\
.0020 \\
.0009 \\
.0004 \\
.0002 \\
.0001 \\
.0000 \\
.0000 \\
.0000 \\
.0000 \\
.0000 \\
.0000 \\
.0000 \\
.0000 \\
.0000 \\
.0000 \\
.0000 \\
.0000 \\
.0000 \\
.0000 \\
.0000 \\
.0000 \\
.0000 \\
.0000\end{array}$ & $\begin{array}{l}.0104 \\
.0389 \\
.0792 \\
.1162 \\
.1379 \\
.1403 \\
.1271 \\
.1051 \\
.0806 \\
.0582 \\
.0398 \\
.0261 \\
.0165 \\
.0000 \\
.0059 \\
.0034 \\
.0019 \\
.0011 \\
.0006 \\
.0003 \\
.0002 \\
.0001 \\
.0000 \\
.0000 \\
.0000 \\
.0000 \\
.0000 \\
.0000 \\
.0000 \\
.0000 \\
.0000 \\
.0000 \\
.0000 \\
.0000 \\
.0000\end{array}$ & $\begin{array}{l}.0023 \\
.0106 \\
.0268 \\
.0492 \\
.0730 \\
.0928 \\
.1051 \\
.1086 \\
.1043 \\
.0939 \\
.0804 \\
.0659 \\
.0519 \\
.0396 \\
.0293 \\
.0212 \\
.0149 \\
.0103 \\
.0070 \\
.0047 \\
.0031 \\
.0020 \\
.0013 \\
.0008 \\
.0005 \\
.0003 \\
.0002 \\
.0001 \\
.0001 \\
.0000 \\
.0000 \\
.0000 \\
.0000 \\
.0000 \\
.0000\end{array}$ & $\begin{array}{l}.0004 \\
.0021 \\
.0064 \\
.0141 \\
.0250 \\
.0382 \\
.0519 \\
.0643 \\
.0740 \\
.0801 \\
.0823 \\
.0809 \\
.0765 \\
.0700 \\
.0622 \\
.0539 \\
.0456 \\
.0378 \\
.0307 \\
.0246 \\
.0194 \\
.0151 \\
.0115 \\
.0088 \\
.0066 \\
.0049 \\
.0036 \\
.0026 \\
.0019 \\
.0014 \\
.0010 \\
.0007 \\
.0005 \\
.0003 \\
.0002\end{array}$ & $\begin{array}{l}.0000 \\
.0003 \\
.0010 \\
.0025 \\
.0051 \\
.0091 \\
.0144 \\
.0208 \\
.0280 \\
.0353 \\
.0424 \\
.0486 \\
.0536 \\
.0572 \\
.0593 \\
.0599 \\
.0592 \\
.0572 \\
.0543 \\
.0507 \\
.0466 \\
.0422 \\
.0378 \\
.0334 \\
.0292 \\
.0253 \\
.0217 \\
.0185 \\
.0156 \\
.0131 \\
.0109 \\
.0090 \\
.0074 \\
.0060 \\
.0049\end{array}$ & $\begin{array}{l}.0000 \\
.0000 \\
.0001 \\
.0002 \\
.0005 \\
.0010 \\
.0018 \\
.0029 \\
.0045 \\
.0065 \\
.0089 \\
.0117 \\
.0147 \\
.0180 \\
.0213 \\
.0246 \\
.0277 \\
.0306 \\
.0332 \\
.0355 \\
.0372 \\
.0386 \\
.0395 \\
.0399 \\
.0399 \\
.0395 \\
.0387 \\
.0377 \\
.0363 \\
.0348 \\
.0331 \\
.0313 \\
.0294 \\
.0274 \\
.0255\end{array}$ & $\begin{array}{l}.0000 \\
.0000 \\
.0000 \\
.0000 \\
.0000 \\
.0000 \\
.0000 \\
.0001 \\
.0001 \\
.0002 \\
.0004 \\
.0005 \\
.0007 \\
.0010 \\
.0014 \\
.0018 \\
.0022 \\
.0028 \\
.0034 \\
.0041 \\
.0048 \\
.0056 \\
.0065 \\
.0074 \\
.0083 \\
.0092 \\
.0102 \\
.0111 \\
.0121 \\
.0130 \\
.01339 \\
.0148 \\
.0156 \\
.0164 \\
.0172\end{array}$ \\
\hline
\end{tabular}


THETA $(\theta)$

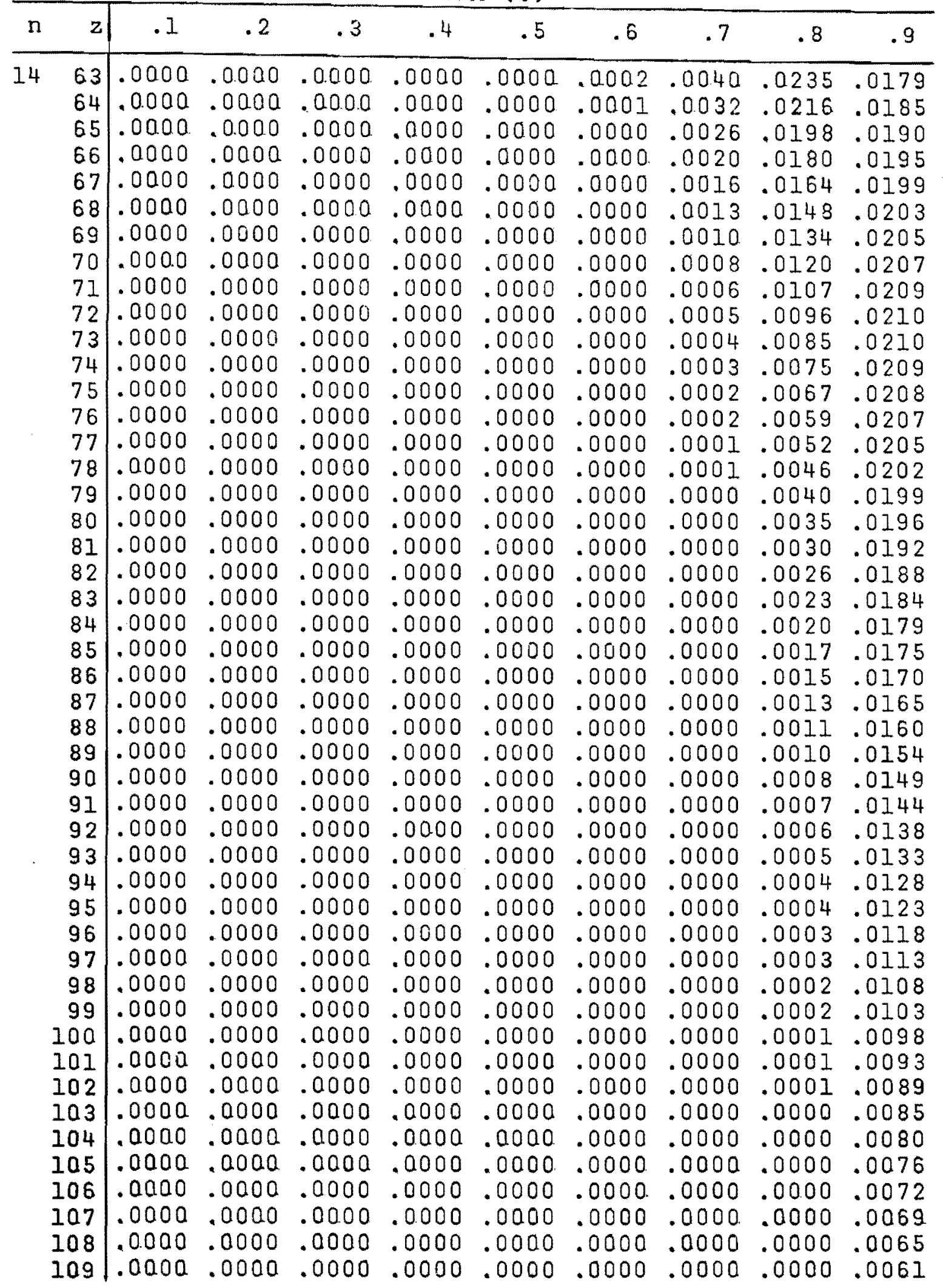


THETA $(\theta)$

\begin{tabular}{|c|c|c|c|c|c|c|c|c|c|c|}
\hline $\mathrm{n}$ & $z$ & 1 & .2 & .3 & .4 & .5 & .6 & .7 & .8 & .9 \\
\hline & $\begin{array}{l}110 \\
111 \\
112 \\
113 \\
114 \\
115 \\
116 \\
117 \\
118 \\
119 \\
120 \\
121 \\
122 \\
123 \\
124 \\
125 \\
126 \\
127 \\
128 \\
129 \\
130 \\
131 \\
132 \\
133 \\
134 \\
135 \\
136 \\
137 \\
138 \\
139 \\
140 \\
141 \\
142 \\
143 \\
144 \\
145 \\
146 \\
147 \\
148 \\
149 \\
150 \\
151 \\
152 \\
153 \\
154 \\
155 \\
156\end{array}$ & $\begin{array}{l}.0000 \\
.0000 \\
.0000 \\
.0000 \\
.0000 \\
.0000 \\
.0000 \\
.0000 \\
.0000 \\
.0000 \\
.0000 \\
.0000 \\
.0000 \\
.0000 \\
.0000 \\
.0000 \\
.0000 \\
.0000 \\
.0000 \\
.0000 \\
.0000 \\
.0000 \\
.0000 \\
.0000 \\
.0000 \\
.0000 \\
.0000 \\
.0000 \\
.0000 \\
.00000 \\
.000000 \\
.00000 \\
.00000 \\
.0000 \\
.0000 \\
.0000 \\
.00000 \\
.0000\end{array}$ & $\begin{array}{l}.0000 \\
.0000 \\
.0000 \\
.0000 \\
.0000 \\
.0000 \\
.0000 \\
.0000 \\
.0000 \\
.0000 \\
.0000 \\
.0000 \\
.0000 \\
.0000 \\
.0000 \\
.0000 \\
.0000 \\
.0000 \\
.0000 \\
.0000 \\
.0000 \\
.0000 \\
.0000 \\
.0000 \\
.0000 \\
.0000 \\
.0000 \\
.0000 \\
.0000 \\
.0000 \\
.0000 \\
.0000 \\
.00000 \\
.000000 \\
.00000 \\
.00000 \\
.00000 \\
.00000 \\
.0000\end{array}$ & $\begin{array}{l}.0000 \\
.0000 \\
.0000 \\
.0000 \\
.0000 \\
.0000 \\
.0000 \\
.0000 \\
.0000 \\
.0000 \\
.0000 \\
.0000 \\
.0000 \\
.0000 \\
.0000 \\
.0000 \\
.0000 \\
.0000 \\
.0000 \\
.0000 \\
.0000 \\
.0000 \\
.0000 \\
.0000 \\
.0000 \\
.0000 \\
.0000 \\
.0000 \\
.0000 \\
.0000 \\
.0000 \\
.0000 \\
.00000 \\
.00000 \\
.0000 \\
.00000 \\
.00000\end{array}$ & $\begin{array}{l}.0000 \\
.0000 \\
.0000 \\
.0000 \\
.0000 \\
.0000 \\
.0000 \\
.0000 \\
.0000 \\
.0000 \\
.0000 \\
.0000 \\
.0000 \\
.0000 \\
.0000 \\
.0000 \\
.0000 \\
.0000 \\
.0000 \\
.0000 \\
.0000 \\
.0000 \\
.0000 \\
.0000 \\
.0000 \\
.0000 \\
.0000 \\
.0000 \\
.0000 \\
.0000 \\
.0000 \\
.0000 \\
.00000 \\
.000000 \\
.00000 \\
.000000 \\
.00000 \\
.00000\end{array}$ & $\begin{array}{l}.0000 \\
.0000 \\
.0000 \\
.0000 \\
.0000 \\
.0000 \\
.0000 \\
.0000 \\
.0000 \\
.0000 \\
.0000 \\
.0000 \\
.0000 \\
.0000 \\
.0000 \\
.0000 \\
.0000 \\
.0000 \\
.0000 \\
.0000 \\
.0000 \\
.0000 \\
.0000 \\
.0000 \\
.0000 \\
.0000 \\
.0000 \\
.0000 \\
.0000 \\
.00000 \\
.000000 \\
.00000 \\
.0000 \\
.00000 \\
.00000 \\
.000000 \\
.00000\end{array}$ & 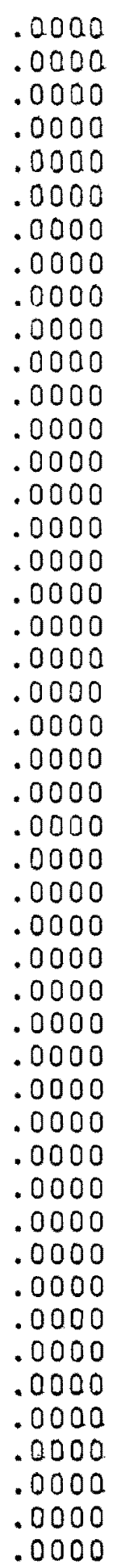 & $\begin{array}{l}0000 \\
0000 \\
0000 \\
0000 \\
0000 \\
0000 \\
0000 \\
0000 \\
0000 \\
0000 \\
0000 \\
0000 \\
0000 \\
0000 \\
0000 \\
0000 \\
0000 \\
0000 \\
0000 \\
0000 \\
0000 \\
0000 \\
0000 \\
0000 \\
0000 \\
0000 \\
0000 \\
0000 \\
0000 \\
0000 \\
0000 \\
0000 \\
0000 \\
0000 \\
0000 \\
0000 \\
0000 \\
0000 \\
0000 \\
0000 \\
0000 \\
0000 \\
0000 \\
0000 \\
0000\end{array}$ & $\begin{array}{l}.0000 \\
.0000 \\
.0000 \\
.0000 \\
.0000 \\
.0000 \\
.0000 \\
.0000 \\
.0000 \\
.0000 \\
.0000 \\
.0000 \\
.0000 \\
.0000 \\
.0000 \\
.0000 \\
.0000 \\
.0000 \\
.0000 \\
.0000 \\
.0000 \\
.0000 \\
.0000 \\
.0000 \\
.0000 \\
.0000 \\
.0000 \\
.0000 \\
.0000 \\
.0000 \\
.0000 \\
.0000 \\
.0000 \\
.0000 \\
.0000 \\
.00000 \\
.000000 \\
.000000 \\
.0000 \\
.0000\end{array}$ & $\begin{array}{l}.0015 \\
.001 \\
.0013 \\
.0012 \\
.0012 \\
.0011 \\
.0010 \\
.0009 \\
.0009 \\
.0008 \\
.000 \\
.000 \\
.0006 \\
.0008 \\
.0006 \\
.000 \\
.000 \\
.000 \\
.000 \\
.000 \\
.0002 \\
.0003 \\
.0003 \\
.000 \\
.000 \\
.000\end{array}$ \\
\hline
\end{tabular}


THETA $(\theta)$

\begin{tabular}{|c|c|c|c|c|c|c|c|c|c|c|}
\hline $\mathrm{n}$ & $\mathrm{z}$ & .1 & .2 & .3 & .4 & .5 & .6 & .7 & .8 & .9 \\
\hline 14 & $\begin{array}{l}157 \\
158 \\
159 \\
160 \\
161 \\
162 \\
163 \\
164 \\
165 \\
166\end{array}$ & $\begin{array}{l}.0000 \\
.0000 \\
.0000 \\
.0000 \\
.0000 \\
.0000 \\
.0000 \\
.0000 \\
.0000 \\
.0000\end{array}$ & $\begin{array}{l}.0000 \\
.0000 \\
.0000 \\
.0000 \\
.0000 \\
.0000 \\
.0000 \\
.0000 \\
.0000 \\
.0000\end{array}$ & $\begin{array}{l}.0000 \\
.0000 \\
.0000 \\
.0000 \\
.0000 \\
.0000 \\
.0000 \\
.0000 \\
.0000 \\
.0000\end{array}$ & $\begin{array}{l}.0000 \\
.0000 \\
.0000 \\
.0000 \\
.0000 \\
.0000 \\
.0000 \\
.0000 \\
.0000 \\
.0000\end{array}$ & $\begin{array}{l}.0000 \\
.0000 \\
.0000 \\
.0000 \\
.0000 \\
.0000 \\
.0000 \\
.0000 \\
.0000 \\
.0000\end{array}$ & $\begin{array}{l}.0000 \\
.0000 \\
.0000 \\
.0000 \\
.0000 \\
.0000 \\
.0000 \\
.0000 \\
.0000 \\
.0000\end{array}$ & $\begin{array}{l}.0000 \\
.0000 \\
.0000 \\
.0000 \\
.0000 \\
.0000 \\
.0000 \\
.0000 \\
.0000 \\
.0000\end{array}$ & $\begin{array}{l}.0000 \\
.0000 \\
.0000 \\
.0000 \\
.0000 \\
.0000 \\
.0000 \\
.0000 \\
.0000 \\
.0000\end{array}$ & $\begin{array}{l}.0002 \\
.0002 \\
.0002 \\
.0002 \\
.0001 \\
.0001 \\
.0001 \\
.0001 \\
.0001 \\
.0000\end{array}$ \\
\hline 5 & $\begin{array}{l}30 \\
31 \\
32 \\
33 \\
34 \\
35 \\
36 \\
37 \\
38 \\
39 \\
40 \\
41 \\
42 \\
43 \\
44 \\
45 \\
46 \\
47 \\
48 \\
49 \\
50 \\
51 \\
52 \\
53 \\
54 \\
55 \\
56 \\
57 \\
58 \\
59 \\
60 \\
61 \\
62 \\
63 \\
64 \\
65\end{array}$ & $\begin{array}{l}.3519 \\
.3519 \\
.1906 \\
.0742 \\
.0232 \\
.0062 \\
.0015 \\
.0003 \\
.0001 \\
.0000 \\
.0000 \\
.0000 \\
.0000 \\
.0000 \\
.0000 \\
.0000 \\
.0000 \\
.0000 \\
.0000 \\
.0000 \\
.0000 \\
.0000 \\
.0000 \\
.0000 \\
.0000 \\
.0000 \\
.0000 \\
.0000 \\
.0000 \\
.0000 \\
.0000 \\
.0000 \\
.0000 \\
.0000 \\
.0000 \\
.0000\end{array}$ & $\begin{array}{l}.1119 \\
.2239 \\
.2425 \\
.1888 \\
.1183 \\
.0633 \\
.0301 \\
.0130 \\
.0052 \\
.0020 \\
.0007 \\
.0002 \\
.0001 \\
.0000 \\
.0000 \\
.0000 \\
.0000 \\
.0000 \\
.0000 \\
.0000 \\
.0000 \\
.0000 \\
.0000 \\
.0000 \\
.0000 \\
.0000 \\
.0000 \\
.0000 \\
.0000 \\
.0000 \\
.0000 \\
.0000 \\
.0000 \\
.0000 \\
.0000 \\
.0000\end{array}$ & $\begin{array}{l}.0314 \\
.0943 \\
.1532 \\
.1789 \\
.1681 \\
.1350 \\
.0962 \\
.0623 \\
.0374 \\
.0211 \\
.0112 \\
.0057 \\
.0028 \\
.0013 \\
.0006 \\
.0003 \\
.0001 \\
.0000 \\
.0000 \\
.0000 \\
.0000 \\
.0000 \\
.0000 \\
.0000 \\
.0000 \\
.0000 \\
.0000 \\
.0000 \\
.0000 \\
.0000 \\
.0000 \\
.0000 \\
.0000 \\
.0000 \\
.0000 \\
.0000\end{array}$ & $\begin{array}{l}.0075 \\
.0301 \\
.0653 \\
.1016 \\
.1273 \\
.1363 \\
.1295 \\
.1119 \\
.0895 \\
.0672 \\
.0478 \\
.0324 \\
.0211 \\
.0133 \\
.0081 \\
.0048 \\
.0028 \\
.0016 \\
.0009 \\
.0005 \\
.0003 \\
.0001 \\
.0001 \\
.0000 \\
.0000 \\
.0000 \\
.0000 \\
.0000 \\
.0000 \\
.0000 \\
.0000 \\
.0000 \\
.0000 \\
.0000 \\
.0000 \\
.0000\end{array}$ & $\begin{array}{l}.0015 \\
.0073 \\
.0198 \\
.0386 \\
.0604 \\
.0808 \\
.0960 \\
.1037 \\
.1037 \\
.0973 \\
.0865 \\
.0734 \\
.0598 \\
.0471 \\
.0359 \\
.0267 \\
.0194 \\
.0137 \\
.0095 \\
.0065 \\
.0044 \\
.0029 \\
.0019 \\
.0012 \\
.0008 \\
.0005 \\
.0003 \\
.0002 \\
.0001 \\
.0001 \\
.0000 \\
.0000 \\
.0000 \\
.0000 \\
.0000 \\
.0000\end{array}$ & $\begin{array}{l}.0002 \\
.0013 \\
.0041 \\
.0097 \\
.0182 \\
.0292 \\
.0417 \\
.0540 \\
.0648 \\
.0730 \\
.0778 \\
.0792 \\
.0775 \\
.0732 \\
.0671 \\
.0598 \\
.0520 \\
.0443 \\
.0370 \\
.0303 \\
.0244 \\
.0194 \\
.0152 \\
.0118 \\
.0090 \\
.0068 \\
.0051 \\
.0038 \\
.0028 \\
.0020 \\
.0015 \\
.0011 \\
.0008 \\
.0005 \\
.0004 \\
.0003\end{array}$ & $\begin{array}{l}.0000 \\
.0001 \\
.0005 \\
.0014 \\
.0032 \\
.0059 \\
.0099 \\
.0150 \\
.0209 \\
.0275 \\
.0342 \\
.0406 \\
.0464 \\
.0511 \\
.0546 \\
.0568 \\
.0577 \\
.0573 \\
.0558 \\
.0533 \\
.0502 \\
.0465 \\
.0425 \\
.0384 \\
.0343 \\
.0303 \\
.0265 \\
.0229 \\
.0197 \\
.0168 \\
.0142 \\
.0119 \\
.0100 \\
.0083 \\
.0068 \\
.0056\end{array}$ & $\begin{array}{l}.0000 \\
.0000 \\
.0000 \\
.0001 \\
.0002 \\
.0005 \\
.0010 \\
.0017 \\
.0027 \\
.0041 \\
.0059 \\
.0079 \\
.0104 \\
.0131 \\
.0159 \\
.0189 \\
.0220 \\
.0249 \\
.0277 \\
.0303 \\
.0326 \\
.0345 \\
.0361 \\
.0373 \\
.0380 \\
.0384 \\
.0384 \\
.0380 \\
.0373 \\
.0363 \\
.0351 \\
.0337 \\
.0321 \\
.0305 \\
.0287 \\
.0269\end{array}$ & $\begin{array}{l}.0000 \\
.0000 \\
.0000 \\
.0000 \\
.0000 \\
.0000 \\
.0000 \\
.0000 \\
.0001 \\
.0001 \\
.0002 \\
.0003 \\
.0004 \\
.0005 \\
.0007 \\
.0010 \\
.0013 \\
.0017 \\
.0021 \\
.0026 \\
.0031 \\
.0037 \\
.0043 \\
.0050 \\
.0058 \\
.0066 \\
.0074 \\
.0082 \\
.0091 \\
.0099 \\
.0108 \\
.0117 \\
.0125 \\
.0133 \\
.0141 \\
.0149\end{array}$ \\
\hline
\end{tabular}


THETA $(\theta)$

\begin{tabular}{|c|c|c|c|c|c|c|c|c|c|}
\hline$n$ & .1 & .2 & .3 & .4 & .5 & .6 & .7 & .8 & .9 \\
\hline $\begin{array}{l}66 \\
67 \\
68 \\
69 \\
70 \\
71 \\
72 \\
73 \\
74 \\
75 \\
76 \\
77 \\
78 \\
79 \\
80 \\
81 \\
82 \\
83 \\
84 \\
85 \\
86 \\
87 \\
88 \\
89 \\
90 \\
91 \\
92 \\
93 \\
94 \\
95 \\
96 \\
97 \\
98 \\
99 \\
100 \\
101 \\
102 \\
103 \\
104 \\
105 \\
106 \\
107 \\
108 \\
109 \\
110 \\
111 \\
112\end{array}$ & $\begin{array}{l}.0000 \\
.0000 \\
.0000 \\
.0000 \\
.0000 \\
.0000 \\
.0000 \\
.0000 \\
.0000 \\
.0000 \\
.0000 \\
.0000 \\
.0000 \\
.0000 \\
.0000 \\
.0000 \\
.0000 \\
.0000 \\
.0000 \\
.0000 \\
.0000 \\
.0000 \\
.0000 \\
.0000 \\
.0000 \\
.0000 \\
.0000 \\
.0000 \\
.0000 \\
.0000 \\
.0000 \\
.0000 \\
.0000 \\
.0000 \\
.0000 \\
.0000 \\
.0000 \\
.0000 \\
.0000 \\
.0000 \\
.0000 \\
.0000 \\
.0000 \\
.0000 \\
.0000 \\
.0000 \\
.0000\end{array}$ & $\begin{array}{l}.0000 \\
.0000 \\
.0000 \\
.0000 \\
.0000 \\
.0000 \\
.0000 \\
.0000 \\
.0000 \\
.0000 \\
.0000 \\
.0000 \\
.0000 \\
.0000 \\
.0000 \\
.0000 \\
.0000 \\
.0000 \\
.0000 \\
.0000 \\
.0000 \\
.0000 \\
.0000 \\
.0000 \\
.0000 \\
.0000 \\
.0000 \\
.0000 \\
.00 \\
.0000 \\
.0000 \\
.0000 \\
.0000 \\
.0000 \\
.0000 \\
.0000 \\
.0000 \\
.0000 \\
.0000 \\
.000\end{array}$ & $\begin{array}{l}.0000 \\
.0000 \\
.0000 \\
.0000 \\
.0000 \\
.0000 \\
.0000 \\
.0000 \\
.0000 \\
.0000 \\
.0000 \\
.0000 \\
.0000 \\
.0000 \\
.0000 \\
.0000 \\
.0000 \\
.0000 \\
.0000 \\
.0000 \\
.0000 \\
.0000 \\
.0000 \\
.0000 \\
.0000 \\
.0000 \\
.0000 \\
.0000 \\
.0000 \\
.00000 \\
.000000 \\
.00000 \\
.00000 \\
.00000 \\
.000000 \\
.00000\end{array}$ & $\begin{array}{l}.0000 \\
.0000 \\
.0000 \\
.0000 \\
.0000 \\
.0000 \\
.0000 \\
.0000 \\
.0000 \\
.0000 \\
.0000 \\
.0000 \\
.0000 \\
.0000 \\
.0000 \\
.0000 \\
.0000 \\
.0000 \\
.0000 \\
.0000 \\
.0000 \\
.0000 \\
.0000 \\
.0000 \\
.0000 \\
.0000 \\
.0000 \\
.0000 \\
.00000 \\
.000000 \\
.00000 \\
.00000 \\
.00000 \\
.00000 \\
.00000 \\
.0000 \\
.0000 \\
.0000\end{array}$ & $\begin{array}{l}.0000 \\
.0000 \\
.0000 \\
.0000 \\
.0000 \\
.0000 \\
.0000 \\
.0000 \\
.0000 \\
.0000 \\
.0000 \\
.0000 \\
.0000 \\
.0000 \\
.0000 \\
.0000 \\
.0000 \\
.0000 \\
.0000 \\
.0000 \\
.0000 \\
.0000 \\
.0000 \\
.0000 \\
.0000 \\
.0000 \\
.0000 \\
.0000 \\
.0000 \\
.0000 \\
.0000 \\
.0000 \\
.0000 \\
.0000 \\
.00000 \\
.0000 \\
.00000 \\
.00000 \\
.00000 \\
.0000\end{array}$ & 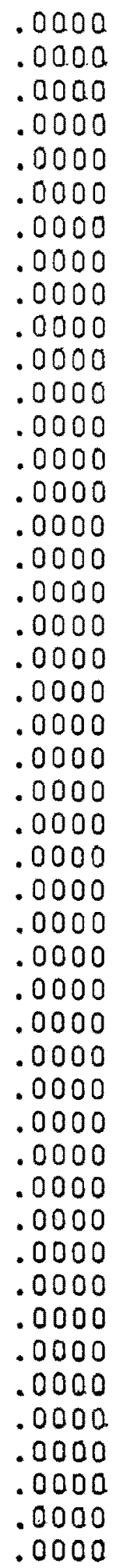 & $\begin{array}{l}.0000 \\
.0000 \\
.0000 \\
.0000 \\
.0000 \\
.0000 \\
.0000 \\
.0000 \\
.0000 \\
.0000 \\
.0000 \\
.0000 \\
.0000 \\
.0000 \\
.0000 \\
.0000 \\
.0000 \\
.0000 \\
.0000 \\
.0000 \\
.0000 \\
.0000 \\
.0000 \\
.0000 \\
.0000 \\
.0000 \\
.0000 \\
.0000 \\
.0000 \\
.00000 \\
.000000 \\
.000000 \\
.000000 \\
.00000 \\
.00000 \\
.00000 \\
.00000\end{array}$ & $\begin{array}{l}.0148 \\
.0134 \\
.0120 \\
.0107 \\
.0096 \\
.0085 \\
.0075 \\
.0067 \\
.0059 \\
.0052 \\
.0046 \\
.0040 \\
.0035 \\
.0030 \\
.0026 \\
.0023 \\
.0020 \\
.0017 \\
.0015 \\
.0013 \\
.0011 \\
.0010 \\
.0008 \\
.0007 \\
.0006 \\
.0005 \\
.0004 \\
.0004 \\
.0003 \\
.0003 \\
.0002 \\
.0002 \\
.0001 \\
.0001 \\
.0001 \\
.0000 \\
.0000 \\
.0000 \\
.0000 \\
.0000 \\
.0000 \\
.0000 \\
.0000 \\
.00000\end{array}$ & $\begin{array}{l}.0191 \\
.0188 \\
.0185 \\
.0181 \\
.0177 \\
.0173 \\
.0169 \\
.0164 \\
.0160 \\
.0155 \\
.0150 \\
.0145 \\
.0140 \\
.0135 \\
.0130 \\
.01126 \\
.01111 \\
.0116 \\
.0111 \\
.0106 \\
.0102 \\
.0097 \\
.0093 \\
.0089 \\
.0085 \\
.0081 \\
.0077 \\
.0073\end{array}$ \\
\hline
\end{tabular}


THETA $(\theta)$

\begin{tabular}{|c|c|c|c|c|c|c|c|c|c|c|}
\hline $\mathrm{n}$ & & .1 & .2 & .3 & .4 & .5 & .6 & .7 & .8 & .9 \\
\hline \multirow[t]{47}{*}{5} & 113 & .0000 & 0000 & 0000 & .0000 & .0000 & .0000 & .0000 & .0000 & $.006 \mathrm{~b}$ \\
\hline & 114 & .0000 & .0000 & .0000 & .0000 & .0000 & .0000 & .0000 & .0000 & \\
\hline & 115 & .0000 & .0000 & .0000 & .0000 & .0000 & .0000 & .0000 & .0000 & .0062 \\
\hline & 116 & .0000 & .0000 & .0000 & .0000 & .0000 & .0000 & .0000 & .0000 & \\
\hline & 117 & .0000 & .0000 & .0000 & .0000 & .0000 & .0000 & .0000 & .0000 & .0056 \\
\hline & 118 & .0000 & .0000 & .0000 & .0000 & .0000 & .0000 & .0000 & .0000 &. \\
\hline & 119 & .0000 & .0000 & .0000 & .0000 & .0000 & .0000 & .0000 & .0000 & .005 \\
\hline & 120 & .0000 & .0000 & .0000 & .0000 & .0000 & .0000 & .0000 & .0000 & .004 \\
\hline & 121 & .0000 & .0000 & .0000 & .0000 & .0000 & .0000 & .0000 & .0000 & .0044 \\
\hline & 122 & .0000 & .0000 & .0000 & .0000 & .0000 & .0000 & .0000 & .0000 & .0042 \\
\hline & 123 & .0000 & .0000 & .0000 & .0000 & .0000 & .0000 & .0000 & .0000 & .0040 \\
\hline & 124 & .0000 & .0000 & .0000 & .0000 & .0000 & .0000 & .0000 & .0000 & .0037 \\
\hline & 125 & .0000 & .0000 & .0000 & .0000 & .0000 & .0000 & .0000 & .0000 & .0035 \\
\hline & 126 & .0000 & .0000 & .0000 & .0000 & .0000 & .0000 & .0000 & .0000 & .0033 \\
\hline & 127 & .0000 & .0000 & .0000 & .0000 & .0000 & .0000 & .0000 & .0000 & .0031 \\
\hline & 128 & .0000 & .0000 & .0000 & .0000 & .0000 & .0000 & .0000 & & .0029 \\
\hline & 129 & .0000 & .0000 & .0000 & .0000 & .0000 & .0000 & .0000 & .0000 & .0027 \\
\hline & 130 & .0000 & .0000 & .0000 & .0000 & .0000 & .0000 & .0000 & .0000 & .0026 \\
\hline & 131 & .0000 & .0000 & .0000 & .0000 & .0000 & .0000 & .00 & .00 & .0024 \\
\hline & 132 & .0000 & .0000 & .0000 & .0000 & .0000 & .0000 & .0000 & .0000 & .0023 \\
\hline & 133 & .0000 & .0000 & .0000 & .0000 & .0000 & .0000 & .00 & .00 & .002 \\
\hline & 134 & .0000 & .0000 & .0000 & .0000 & .0000 & .0000 & .0000 & .0000 & .0020 \\
\hline & 135 & .0000 & .006 & & .0000 & & .00 & .0000 & & 119 \\
\hline & 136 & .0000 & .0000 & .0000 & .0000 & .0000 & .0000 & .0000 & .00 & .0017 \\
\hline & 137 & .0000 & .0000 & .0000 & .0000 & .0000 & .0000 & .0000 & .00 & .0016 \\
\hline & 138 & .0000 & .0000 & .0000 & .0000 & .0000 & .0000 & .0000 & .0 & .0015 \\
\hline & 139 & .0000 & .0000 & .0000 & .0000 & .0000 & .0000 & .0000 & .00 & .0014 \\
\hline & 140 & .0000 & .0000 & .0000 & .0000 & .0000 & .0000 & $.0 C$ & & .0013 \\
\hline & 147 & .0000 & .0000 & .0000 & .0000 & .0000 & .0000 & .0000 & .0000 & .0012 \\
\hline & 142 & & .00 & & & & & & & \\
\hline & 143 & .0000 & .0000 & .0000 & .0000 & .0000 & .0000 & .0000 & .0 & 11 \\
\hline & 144 & & & & & & & & & \\
\hline & 14 & .0000 & .0 & 00 & .0000 & .00 & .0 & .00 & 0 & 09 \\
\hline & & 100 & & & .0000 & & .00 & .00 & & .0 \\
\hline & 14 & .00 & .0 & .0 & .0000 & .00 & .00 & .0 & .0 & \\
\hline & 14 & .0000 & .0000 & .0000 & .0000 & .00 & .0000 & .00 & & .0008 \\
\hline & 149 & .00 & .0 & .00 & .0000 & .00 & .00 & & & \\
\hline & & .0000 & .0000 & .0000 & .0000 & .0000 & .0000 & .00 & & .0007 \\
\hline & 15 & .0000 & 0000 & 0000 & .0000 & .0000 & 0000 & & & \\
\hline & 15 & 00 & 0000 & 0000 & .0000 & 0000 & 00 & .00 & & .0 \\
\hline & 15 & 0000 & 0000 & .0000 & .0000 & .0000 & .0000 & .00 & & \\
\hline & & 00 & .0000 & .0000 & .0000 & .0000 & .0000 & .0000 & .0000 & .0005 \\
\hline & 75 & 0 & 0000 & 0000 & .0000 & .00 & .00 & & & .00 \\
\hline & & .0000 & .0000 & .0000 & .0000 & .0000 & .0000 & .0000 & .0000 & .0004 \\
\hline & & & & .0000 & .0000 & .00 & .00 & & & .00 \\
\hline & & & .0000 & .0000 & .0000 & & .00 & & .0000 & \\
\hline & & & & & & & & & & .0003 \\
\hline
\end{tabular}


THETA ( $\theta$ )

\begin{tabular}{cr|ccccccccc}
\hline $\mathrm{n}$ & $\mathrm{z}$ & .1 & .2 & .3 & .4 & .5 & .6 & .7 & .8 & .9 \\
\hline 15 & 160 & .000 .0 & .0000 & .0000 & .0000 & .0000 & .0000 & .0000 & .0000 & .0003 \\
& 161 & .0000 & .0000 & .0000 & .0000 & .0000 & .0000 & .0000 & .0000 & .0003 \\
162 & .0000 & .0000 & .0000 & .0000 & .0000 & .0000 & .0000 & .0000 & .0003 \\
163 & .0000 & .0000 & .0000 & .0000 & .0000 & .0000 & .0000 & .0000 & .0002 \\
164 & .0000 & .0000 & .0000 & .0000 & .0000 & .0000 & .0000 & .0000 & .0002 \\
165 & .0000 & .0000 & .0000 & .0000 & .0000 & .0000 & .0000 & .0000 & .0002 \\
166 & .0000 & .0000 & .0000 & .0000 & .0000 & .0000 & .0000 & .0000 & .0002 \\
167 & .0000 & .0000 & .0000 & .0000 & .0000 & .0000 & .0000 & .0000 & .0002 \\
168 & .0000 & .0000 & .0000 & .0000 & .0000 & .0000 & .0000 & .0000 & .0002 \\
169 & .0000 & .0000 & .0000 & .0000 & .0000 & .0000 & .0000 & .0000 & .0001 \\
170 & .0000 & .0000 & .0000 & .0000 & .0000 & .0000 & .0000 & .0000 & .0001 \\
171 & .0000 & .0000 & .0000 & .0000 & .0000 & .0000 & .0000 & .0000 & .0001 \\
172 & .0000 & .0000 & .0000 & .0000 & .0000 & .0000 & .0000 & .0000 & .0001 \\
173 & .0000 & .0000 & .0000 & .0000 & .0000 & .0000 & .0000 & .0000 & .0001 \\
174 & .0000 & .0000 & .0000 & .0000 & .0000 & .0000 & .0000 & .0000 & .0000 \\
\end{tabular}




\section{BIBLIOGRAPHY}

[1] Burington, R. S., and May, D. C. Handbook of Probability and Statistics. Sandusky, ohio: Handbook Publishers, Inc., 1958.

[2] Fisz, M. Probability Theory and Mathematical Statistics. Third edition. New York: John Wiley and Sons, 1963.

[3] Jordan, C. Calculus of Finite Differences. Third edition. New York: Chelsea Publishing Company, 1965.

[4] Patil, G. P., and Wani, J. K. "On Certain Structural Properties of the Logarithmic Series Distribution and the First Type Stirling Distribution." Sankhyā, Series A, 27, 1965, pp. 271-280.

[5] Patil, G. P., Kamat, A. R., and Wani, J. K. Certain Studies on the Structure and Statistics of the Logarithmic Series Distribution and Related Tables. Ohio: Aerospace Research Laboratories, wrightPatterson Air Force Base, 1964. 